\title{
Intrinsic Meta-Modeling of Systems of Systems: A Study in Bridge Infrastructure Management
}

\author{
A Dissertation \\ Presented to \\ The faculty of the School of Engineering and Applied Science \\ University of Virginia
}

\author{
In partial fulfillment \\ of the requirements for the degree \\ Doctor of Philosophy \\ by
}

Eva Andrijcic

August, 2013 
Approval Sheet

This Dissertation

is submitted in partial fulfillment of the requirements for the degree of Doctor of Philosophy

Eva Andrijcic

AUTHOR

The dissertation has been read and approved by the examining committee:

Dr. Yacov Y. Haimes, SIE

Advisor

Dr. Barry Horowitz, SIE

Dr. William Scherer, SIE

Dr. Gerard Learmonth, SIE

Dr. Steven Chase, CEE

Dr. Garry Jacyna, MITRE 


\section{Table of Contents}

\section{Table of Contents}

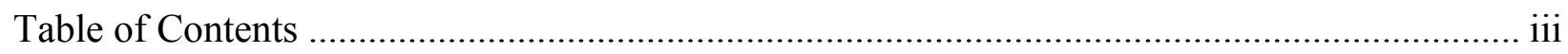

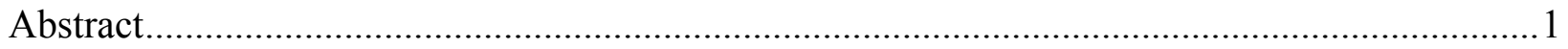

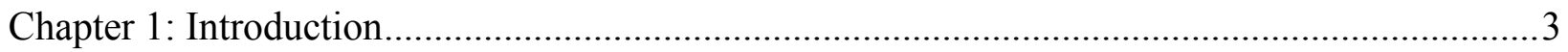

1.1 The U.S. transportation infrastructure: A crumbling system of systems ..............................

1.2 The current condition of the U.S. bridge infrastructure system of systems ..........................5

1.3 The existing disjoint approach to bridge infrastructure management ..................................

1.4 Overcoming impediments to a sustainable bridge infrastructure management: A holistic approach to the modeling and management of bridge infrastructure system of systems ............. 8

1.5 Bridge infrastructure management as part of a larger problem ........................................10

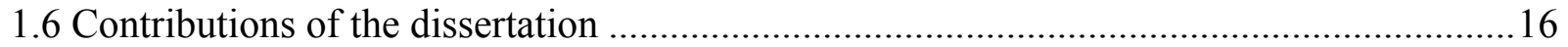

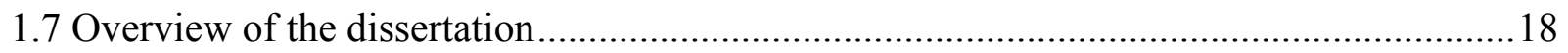

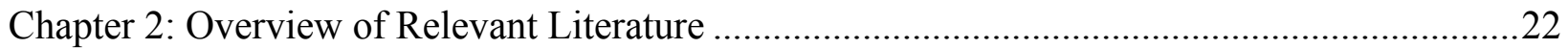

2.1 Literature on modeling systems of systems.................................................................22

2.2 Literature on coordination approaches for systems of systems .........................................2.

2.3 Literature on existing bridge maintenance practices .............................................................30

2.4 Literature on assessing the socio-economic impacts of transportation projects...................32

Chapter 3: Modeling Systems of Systems Through Their Shared States: A Demonstration With a

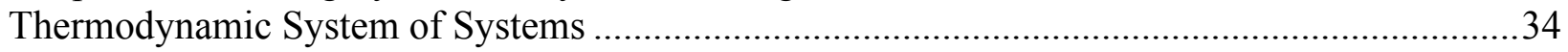

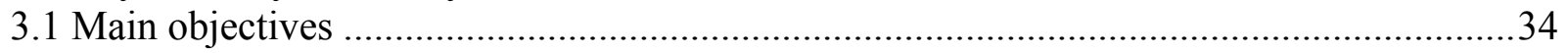

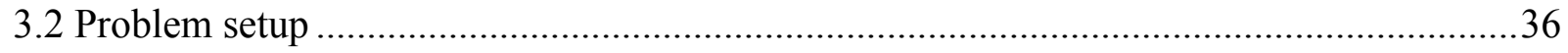

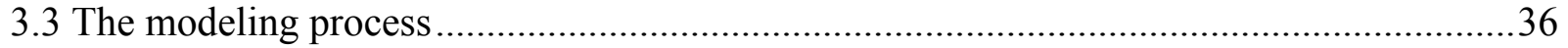

3.4 Implications for larger and more complex systems of systems........................................45

Chapter 4: Methodological Process for Intrinsic Meta-Modeling and for Collaborative

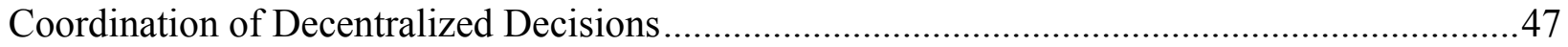

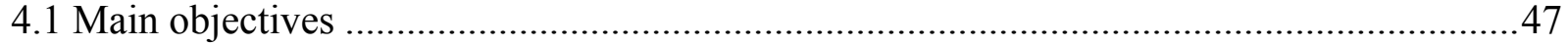

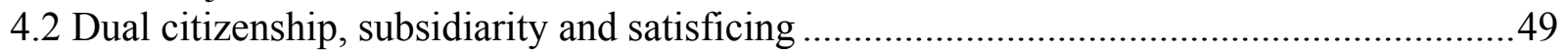

4.3 A need for collaborative coordination through shared state variables ...............................51

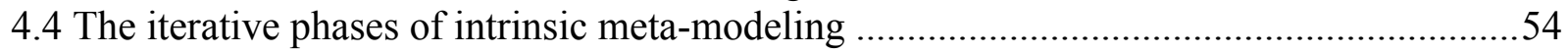

4.4.1 Identifying essential modeling perspectives and questions .............................................56

4.4.2 Identifying representative sub-models, shared and non-shared variables ........................57

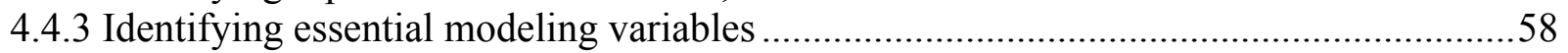

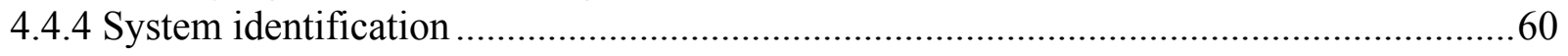

4.4.5 Continuous re-evaluation.....................................................................................6 
4.5 Intrinsic meta-modeling coordination

5. Application of Intrinsic Meta-Modeling to a Bridge Infrastructure System of Systems ..........75

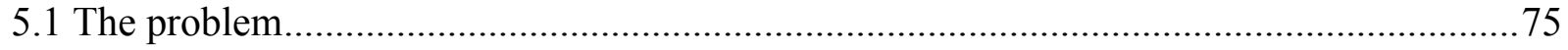

5.2 The strategic importance of the Hampton Roads Bridge Tunnel (HRBT).......................77

5.3 Modeling the bridge infrastructure as a system of systems ...........................................8

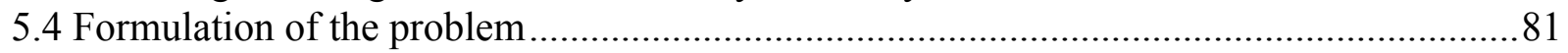

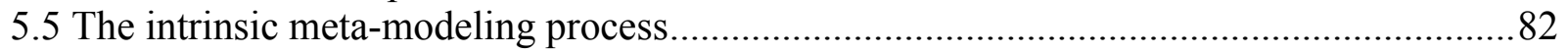

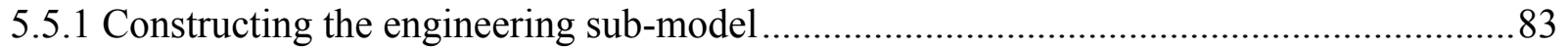

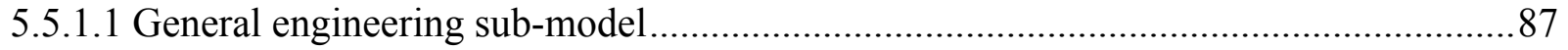

5.5.1.2 Computing parameters of the engineering sub-model.............................................90

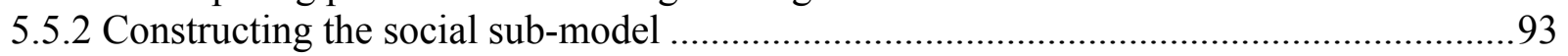

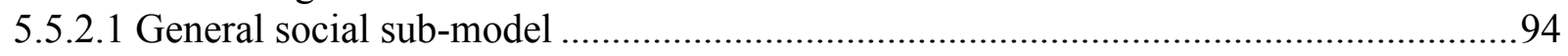

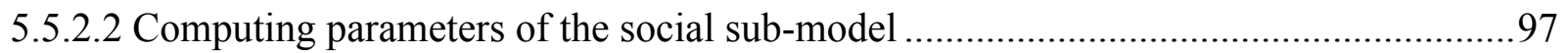

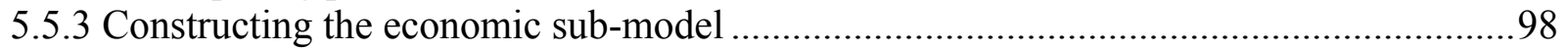

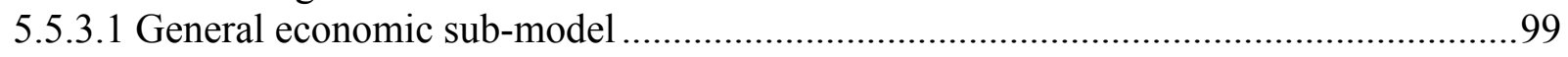

5.5.3.2 Computing parameters of the economic sub-model ............................................... 101

5.5.4 Harmonizing the three sub-models into a meta-model through the introduction of a

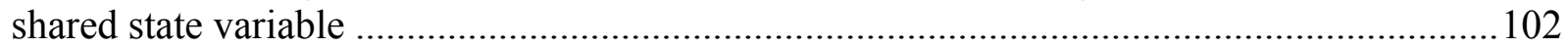

5.6 Traditional solution without collaborative meta-model coordination ............................108

5.7 Holistic solutions with collaborative meta-model coordination.................................... 109

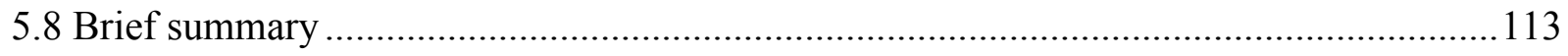

Chapter 6: Broader Public Policy Considerations ................................................................. 115

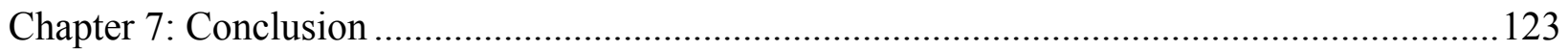

Appendix I: Derivation of a First Principles Model For the Two-Component Thermodynamic

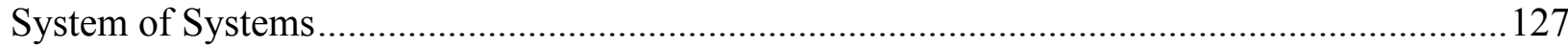

Appendix II: Review of Statistical Performance Criteria..................................................... 136

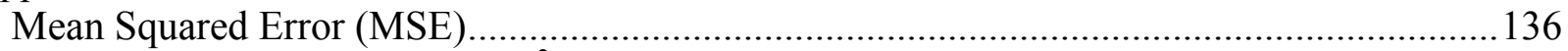

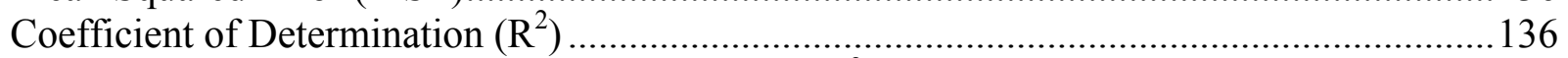

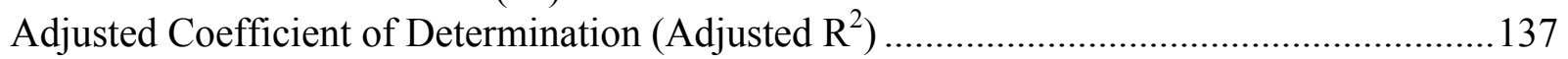

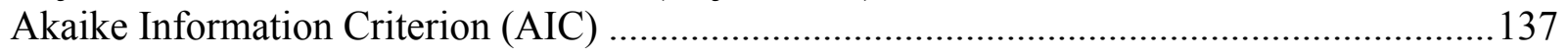

Bias Corrected Akaike Information Criterion (AICc) .............................................. 138

Bayesian Information Criterion (BIC) ................................................................ 138

Appendix III: Bridge System of Systems Meta-Model Supporting Databases .........................139

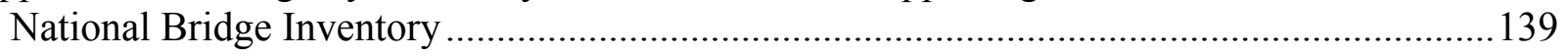

National Climatic Data Center ................................................................................. 139

Safe Accountable Flexible Efficient Transportation Equity Act (SAFETEA) Funding Tables 


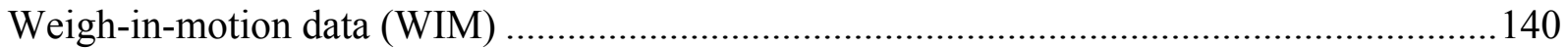

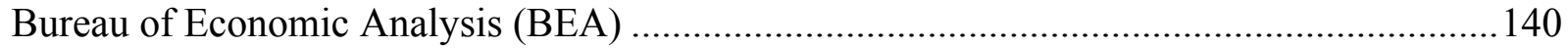

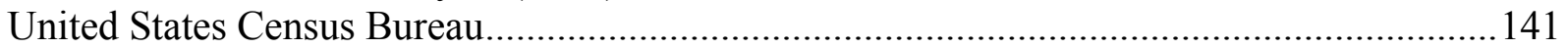

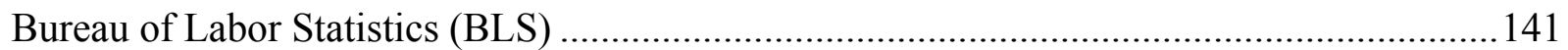

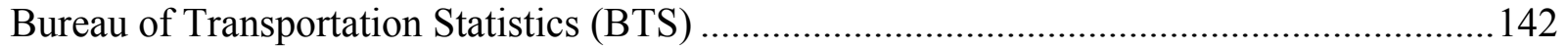

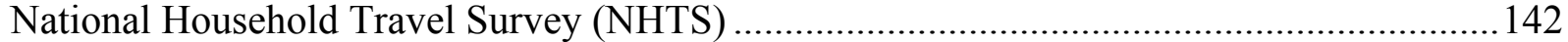

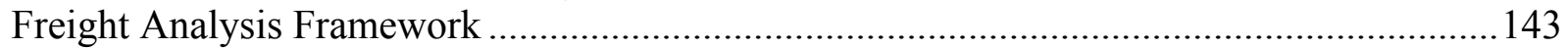

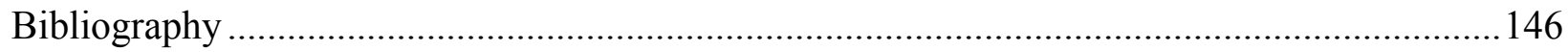




\section{Abstract}

This dissertation builds on the premise that a system of systems must be modeled through multiple models to account for the multifarious perspectives and its sub-systems. The stakeholders associated with the various sub-systems can be managerially and operationally independent, resulting in a distributed and decentralized decision-making environment. The outcomes of the decentralized and interdependent decisions made in a single sub-system affect the states and objectives of the other interdependent sub-systems. To address this challenge, this dissertation presents a modeling framework that builds on the theory of Phantom System Models (PSM) intrinsic meta-modeling to enable the modeling and management of systems of systems through a multi-phase, multi-level, iterative and collaborative coordination process.

The contributions of this dissertation are threefold. First, it introduces an iterative, learn-asyou-go methodological process for the construction of cross-disciplinary models that represent different perspectives of a system of systems, and for their integration into a single meta-model through the use of shared states. Second, it provides theoretical discussion on the use of the intrinsic meta-model as an instrument for collaborative coordination of distributed and decentralized sub-systems' decisions. Intrinsic meta-modeling coordination is defined as the iterative process for adjusting individual sub-system's decisions and constraints in response to measurable and/or observable changes in shared states. Ideally, acceptable levels of shared states can be achievable by all stakeholders over the planning time horizon, while satisficing subsystems' local level objectives. Third, it demonstrates the viability of the developed theory and methodology in its application to the bridge infrastructure system of systems to examine the engineering and socio-economic implications of untimely or insufficient bridge maintenance. It is expected that the developed theory and methodology can be applied to other civil infrastructure systems of systems, thus addressing the recognized need for such approaches.

Other theoretical and practical implications associated with the developed modeling framework include the following: 
(i) The developed process enables and motivates stakeholders who work in a decentralized fashion to identify, quantify, and explicitly model some common and conflicting interests and needs. The ultimate goal of the stakeholders is to adhere to satisficing policies that would be superior to competitive and un-coordinated policies made at the sub-system level without the meta-modeling contribution.

(ii) Given the evolving nature of objectives, interest groups, organizational, political and budgetary baselines, and requirements associated with interdependent sub-systems, the metamodel coordination assumes an even broader role by enabling all stakeholders and decision makers to plan for future emergent changes through collaborative and foresighted efforts. This would yield beneficial outcomes for all concerned parties, and distribute risk ownership among all involved stakeholders. 


\section{Chapter 1: Introduction}

\subsection{The U.S. transportation infrastructure: A crumbling system of systems}

The need to better understand and prioritize the current maintenance needs of the U.S. transportation infrastructure is evidenced in reports spanning the last two and a half decades. As early as 1988, a national commission issued a report titled "Fragile Foundations," citing over 100,000 bridges that were not meeting safety standards (National Council on Public Works Improvement, 1988). This sentiment was recently revisited in reports issued by the American Society of Civil Engineers (ASCE), including a report titled "Can We Come Back From the Brink," which concludes that the U.S. surface transportation infrastructure is "failing to keep pace with the expanding needs of a burgeoning population" (ASCE, 2009b). Similarly Caldwell (2011) suggests that "the quality of America's infrastructure is barely adequate to fulfill current requirements, and insufficient to meet the demands of future economic growth and development." In a series of ongoing civil infrastructure report cards ASCE presents a sober picture of the nation's infrastructures, highlighting the "fragile foundations" of the infrastructures as evidence that citizens and governing bodies are increasingly recognizing the desperate state of our nation's infrastructure systems (ASCE, 1998, 2003, 2005, 2009a, 2013). The most recent issue of the ASCE Infrastructure Report Card gives America's civil infrastructures a grade D+ (poor), and estimates that a $\$ 3.6$ trillion investment will be required by 2020 to bring the U.S. infrastructure systems to an acceptable condition, of which $\$ 1.7$ trillion will be needed to improve the condition of the surface transportation infrastructure (including roads and bridges) (ASCE, 2013). Of the estimated $\$ 1.7$ trillion need, only half will be covered by traditional funding sources, thus leaving a funding gap of approximately $\$ 850$ billion for the surface transportation infrastructure alone.

There are several reasons that have led to the existing condition of the U.S. transportation infrastructure, among which is the fact that the federal investment in the U.S. transportation infrastructure as a percent of GDP has not increased since the late 1960s, when the U.S. 
economy was considerably smaller (Building America's Future Educational Fund, 2011). Currently the U.S. spends less than 2 percent of its GDP on transportation infrastructure, while it spends nearly 5 percent of its GDP on national defense, causing it to be consistently ranked towards the very bottom with respect to 'transportation infrastructure spending as percentage of GDP' among the countries in the Organisation for Economic Co-operation and Development (OECD) (Strauss, 2012). While the federal expenditures for the surface transportation infrastructure (including roads, bridges, highways, transit and rail) account for the largest component of the federal capital infrastructure spending, they fall flat against the projected investment need to bring these assets to an acceptable condition in the near future (Strauss, 2012; ASCE, 2013). Furthermore, infrastructure investments over the last few decades have been primarily dedicated to building new transportation assets, and have often neglected existing roads and bridges that serve as major sources of congestion. The ensuing freight congestion is a serious problem resulting in costs of about $\$ 200$ billion or $1.6 \%$ of the U.S. GDP a year (Building America's Future Educational Fund, 2011).

The practice of persistent infrastructure underinvestment, coupled with a significant growth in commercial and non-commercial transportation demand, has left U.S. transportation infrastructure "stuck in the last century and ill-equipped for the demands of a churning global economy [emphasis added]" (Building America's Future Educational Fund, 2011). ASCE estimates that given the existing transportation infrastructure conditions and investment patterns, by 2040: (i) U.S.'s infrastructure deficiencies will cost the national economy over 400,000 jobs, (ii) American firms will be generating \$232 billion less in value added than they would have if supporting infrastructure had been adequate, and (iii) Americans will be earning $\$ 252$ billion less than would have been possible if supporting infrastructure had been adequate (ASCE, 2011). Business sectors that will be most affected by the deterioration of the transportation infrastructure will include high-value knowledge-based professions, business and medical sectors, and restaurant and entertainment sectors (ASCE, 2011). 


\subsection{The current condition of the U.S. bridge infrastructure system of systems}

Of particular interest in this dissertation is the exploration of the broader effects of the insufficient and untimely maintenance of the U.S. bridge infrastructure. The most recent ASCE report card gives the U.S. bridge infrastructure a grade $\mathrm{C}+$ (mediocre), and it notes that of more than 600,000 bridges in the U.S. approximately $25 \%$ are rated as functionally obsolete or structurally deficient, with an average bridge age of 42 years. Despite recent successes in reducing the overall number of deficient and obsolete bridges, ASCE (2013) indicates, "those bridges that remain classified as structurally deficient are significant in size and length, while the bridges that are being repaired are smaller in scale." The same report furthermore suggests that:

Federal, state, and local bridge investments are not keeping pace with the growing costs of aging bridges. The FHWA estimates that the current cost to repair or replace only the deficient bridges eligible under the Federal Highway Bridge Program is almost $\$ 76$ billion. This total is up from 2009, when FHWA estimated that the total cost was $\$ 71$ billion. If bridge maintenance continues to be deferred over the next 25 years, these backlog costs will rise. To put these numbers in perspective, over the last 30 years Congress has provided approximately $\$ 77$ billion to the states through the federal-aid bridge program. ... The investment backlog for the nation's bridges is estimated to be $\$ 121$ billion, according to FHWA. This figure represents all costbeneficial bridge needs, not just the replacement or rehabilitation of eligible deficient bridges. The $\$ 121$ billion estimate includes $\$ 102$ billion in investment needs for federal-aid highway bridges. Of that $\$ 102$ billion in federal highway needs, $\$ 60$ billion is for the National Highway System bridges, which in turn includes $\$ 38$ billion for Interstate System bridges. The Federal Highway Administration estimates that to eliminate the bridge backlog by 2028 , the nation would need to invest $\$ 20.5$ billion annually; however, at this time only $\$ 12.8$ billion is being spent annually on the nation's bridges. (ASCE, 2013) 
The report concludes by suggesting that the "health of our nation's bridges is directly tied to the nation's ability to compete in a global marketplace" and by suggesting that the bridge maintenance activities should be focused on urban bridges which bear the majority of nation's traffic and "whose upkeep has been consistently deferred due to the significant cost" (ASCE, 2013).

The lack of political will to allocate the needed funds to bridge infrastructure improvement stems, in part, from the disharmonious goals and objectives among the various stakeholders, and political and other decision makers, as well as the lack of appreciation of the critical linkages and interdependencies among the myriad sub-systems of the bridge infrastructure. In order to improve the existing condition of the bridge infrastructure and ensure its viability under increased future demand, bigger maintenance budgets alone will not suffice.

What is needed instead is a more sustainable and proactive bridge management approach that better addresses the existing gap between short-term commitments and long-term needs, and that overcomes the historical impediments to infrastructure condition improvements, including political structure and influences, the lack of public accountability, and a poor understanding of the causal socio-economic effects caused by bridge infrastructure failures. Thus, a significant improvement in the condition of the U.S. bridge infrastructure will have to be founded on a major paradigm shift, so as to enable decision makers and stakeholders to approach the problem from a system of systems perspective, understanding that any viable and sustainable solution must be grounded on engineering, scientific, social, economic and normative contexts (Figure 1). This paradigm shift needs to happen now rather than later, because "although repairing and modernizing the country's infrastructure may seem daunting in lean times, the cost of doing nothing will be exponentially greater" (Caldwell, 2011).

With this in mind, this dissertation presents a system of systems modeling approach (described in detail in Chapters 4 and 5), which enables the harmonization of multiple models, each representing a different sub-system and perspective of a system of systems. The developed approach provides diverse decision makers with the ability to better visualize and collaboratively coordinate their shared and conflicting interests with the purpose of achieving solutions that are more acceptable and sustainable from a holistic system of systems perspective. 


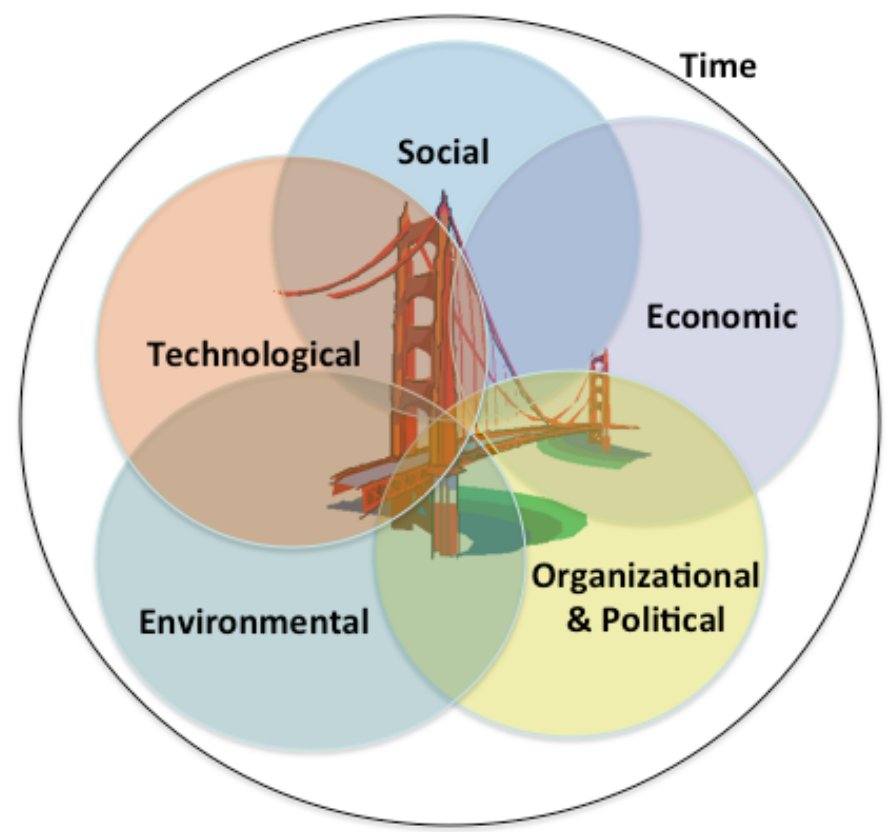

Figure 1: A holistic (gestalt) view of the bridge infrastructure system of systems captures multiple perspectives of the bridge infrastructure management problem

\subsection{The existing disjoint approach to bridge infrastructure management}

Bridge management practices have evolved considerably in the U.S. over the last few decades, and many decision support tools have been developed to enable a more systematic management approach. Decision-makers involved in bridge management nowadays rely heavily on Bridge Management Systems (BMS) to guide them through the various bridge lifecycle stages: design, construction, operation, and maintenance. One of the most commonly used BMS, Pontis, is available through the American Association of State Highway and Transportation Officials (AASHTO), and was created to maintain up-to-date bridge inventories and inspection records, create maintenance and rehabilitation plans, and to systematically and optimally allocate limited resources to maintenance projects (Robert et al., 2003). While Pontis and other BMS have provided the much-needed framework for a systematic evaluation of bridge maintenance needs, these tools are primarily concerned with technical and cost considerations, and they generally ignore considerations like socioeconomic factors, strategic or historical importance, that could influence the choice of most adequate bridge maintenance plan over a long planning horizon. In 
one word, they fail to provide a mechanism for a systemic and holistic management process that accounts for perspectives of all relevant stakeholders.

Traditionally, the process of maintaining the bridge infrastructure has been fairly reactive and non-reflective. Typically, bridge maintenance decisions are often not made until significant structural failures occur, and fixes are often short-term and exclude a serious consideration of the impact of current decisions on: (i) future maintenance options, and (ii) other stakeholders (e.g., commercial and non-commercial infrastructure users). At the highest level the main players in the process include: (i) federal, state, and local level policy makers who determine the amount of funds available for maintenance projects, and (ii) decision makers from Departments of Transportation (DOTs) or Metropolitan Planning Organizations (MPOs), who determine what maintenance will be performed on assets in order to address the most immediate problems, while staying within the constraints of the limited budgets.

Typically in this singular and operationally focused process, decision makers are guided and informed by traditional engineering models, which often rely heavily on subjective and nonrepeatable inspection observations (although efforts are presently under way to design data collection efforts that would collect more informative data in a systematic, repeatable, nonsubjective matter). The general disregard for the potential impacts of current decisions on future maintenance options, and on other stakeholders, often reduces the set of available future maintenance alternatives because the structural condition worsens to the point that only more aggressive maintenance efforts are feasible.

\subsection{Overcoming impediments to a sustainable bridge infrastructure management: A} holistic approach to the modeling and management of bridge infrastructure system of systems

In order to overcome the current impediments to proactive and sustainable bridge infrastructure management a more forward-looking and collaborative modeling and management approach is needed -- one that recognizes that bridge infrastructure systems are more than just built constructs, and one that accounts for the diversity of system stakeholders and their changing requirements, objectives, and constraints. 
Hence, in this dissertation bridge infrastructure is modeled as a system of systems, composed not only of physical sub-systems (i.e., the engineered components), but also of sub-systems that reflect the socio-economic and political environment within which the infrastructure operates (i.e., federal, state, and local level policy makers and decision makers, regional industries, and non-commercial infrastructure users). This representation is appropriate given that these stakeholders frame the extent of possible solutions in bridge infrastructure maintenance and management.

These diverse stakeholders are naturally concerned with different aspects of the bridge infrastructure, and they make decisions in a decentralized and distributed manner, often not realizing the impact that their own decisions have on the interdependent sub-systems. Consequently, this dissertation envisions a forward-looking and collaborative management framework for the bridge infrastructure that would transform the currently singular and nonreflective approach into a multi-disciplinary strategic vision through which decision makers could assess, in a holistic and systematic way, how the maintenance choices they make today affect their future maintenance options, as well as the other relevant stakeholders. Within this framework, infrastructure users could evaluate the impacts that bridge maintenance decisions (or the lack thereof) could have on them, given their changing needs and constraints. Maintenance plans resulting in severe consequences to stakeholders could be collaboratively re-evaluated among all relevant stakeholders by considering, among others, whether: (i) other, less damaging alternatives exist, (ii) innovative funding mechanisms (partially supported by infrastructure users) could be designed to support alternative projects, and (iii) consequences to infrastructure users could be temporarily assuaged in lite of unavoidable projects.

Such an approach theoretically presents several improvements over the traditional approach. It: (i) models a bridge infrastructure as a system of systems, inclusive of the built constructs, as well as the environment within which it operates; (ii) it requires decision makers to assess the broader impacts of current decisions on future options and on involved stakeholders; (iii) introduces a level of transparency, by enabling stakeholders to visualize many common and competing objectives and constraints; and (iv) requires a collaborative effort in achieving global system of systems objectives that benefit all stakeholders, in turn increasing the level of accountability that the general population (i.e., infrastructure users) has for infrastructure 
management, and thus transferring risk ownership among all involved stakeholders. Klosterman (1999) emphasizes the need for collaborative planning approaches for public infrastructures by suggesting that "planning and policy making should not be based on closed and unsupervised "objective" analysis of technical experts," and by stating that "planners are increasingly abandoning the attempts to "plan for" the public for more collaborative efforts to "plan with" the public." To support the development and implementation of such an approach, it is critical to adopt a system of systems modeling process.

\subsection{Bridge infrastructure management as part of a larger problem}

Managing any system necessitates the manipulation of certain system variables in order to achieve a desired outcome (objective), where the functional mappings between system variables and objectives are derived through mathematical modeling. "Simple" systems can be defined as bounded, not always closed entities composed of "interacting components with inputs and outputs from the system's environment" (PlanetMath, 2013). We add the further distinction that all components of a "simple system" are contained within a single circle of concern and influence shown in Figure 2, implying that there is a single context (perspective) through which to model the system. In contrast, in systems of systems components of different sub-systems can be contained in multiple yet overlapping circles of concern and influence shown in Figure 3, thus requiring multiple models that reflect the varied system components and perspectives.

In a model of a "simple" system shown in Figure 2, inputs are related to outputs through state, random, exogenous, and decision variables. With respect to Figure 2, Haimes and Schneiter (1996) suggest that: (i) the system's boundary defines the context within which the system operates, (ii) the circle of concern defines the boundary within which this system is studied, (iii) the circle of influence includes elements that are under the control of a decision maker, (iv) state variables, which are critical in describing the state of the system at any point in time, are of primary concern although decision makers do not always have absolute control over them, (v) random and exogenous variables and constraints are beyond the control of a decision maker, although they are critical in determining the feasible outcomes of a system, and (vi) decision variables are the only variables within a decision maker's circle of influence. 


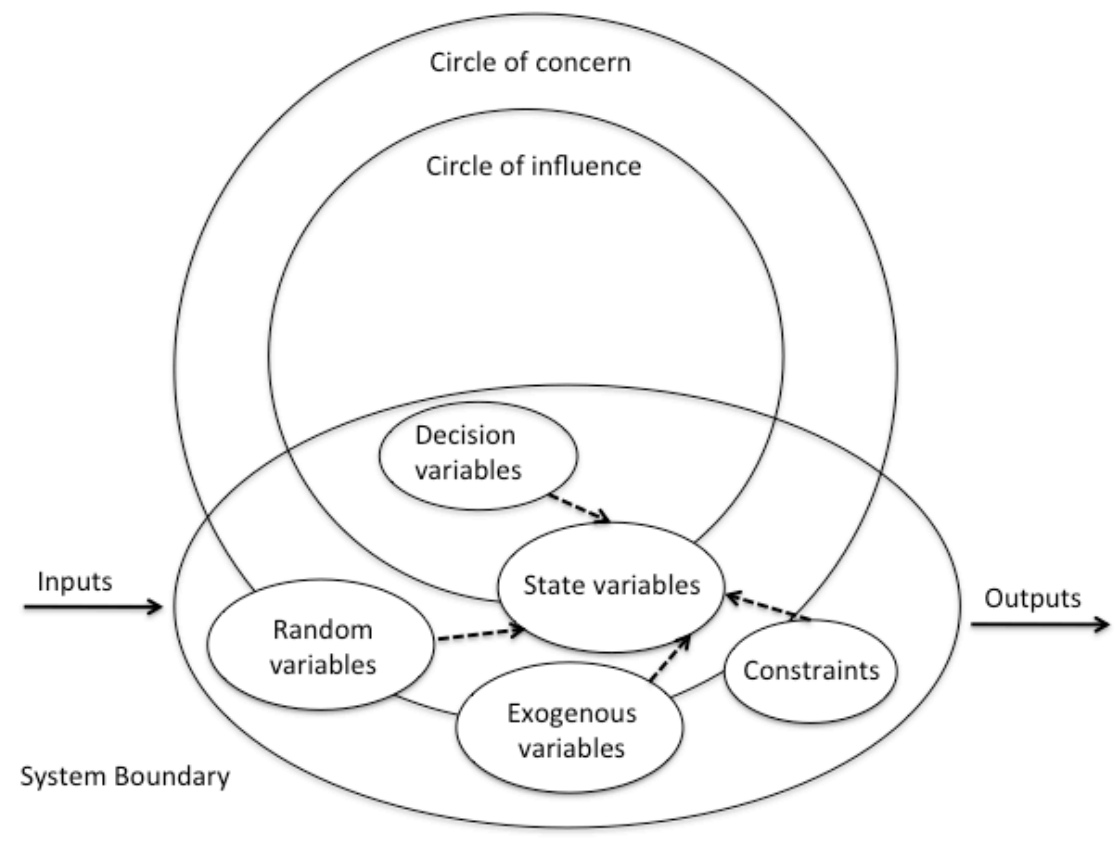

Figure 2: A model of a "simple" system (adapted from Haimes and Schneiter, 1996)

Mathematically Figure 2 is represented as follows:

$\bar{s}(t)=f(\bar{u}(t), \bar{r}(t), \bar{e}(t), \bar{c}(t))$

$\bar{y}(t)=f(\bar{u}(t), \bar{s}(t))$

where,

$$
\begin{aligned}
& C_{C}(t)=f\left(\bar{u}_{\text {decision }}(t), \bar{s}(t),\left\{r_{i}(t) \mid r_{i}(t) \in \bar{r}(t)\right\},\left\{e_{i}(t) \mid e_{i}(t) \in \bar{e}(t)\right\},\left\{c_{i}(t) \mid c_{i}(t) \in \bar{c}(t)\right\}\right) \\
& C_{I}(t)=f\left(\bar{u}_{\text {decision }}(t),\left\{s_{i}(t) \mid s_{i}(t) \in \bar{s}(t)\right\}\right)
\end{aligned}
$$

In Eqs. 1-1a-d, $\bar{y}(t)$ represents a vector of outputs of the system at time $t$, $\bar{u}(t)=\left(\bar{u}_{\text {input }}(t), \bar{u}_{\text {decision }}(t)\right)$ represents a vector of inputs to and decisions made in the system at time $t, \bar{s}(t)$ represents a vector of system states at time $t$, and $\bar{r}(t), \bar{e}(t), \bar{c}(t)$ represent vectors of 
random variables, exogenous variables, and constraints at time $t$, respectively. Furthermore, $C_{C}(t)$ denotes the circle of concern at time $t$, while $C_{I}(t)$ denotes the circle of influence at time $t$. As can be seen from Eqs. 1-1a-d, a "simple" system is characterized by a $\operatorname{single~} C_{C}$ that is a function of decision and state variables, as well as of subsets of random and exogenous variables and constraints, as well as by a single $C_{I}$ that is a function of all decision variables and a subset of state variables. The dependence of $C_{C}$ and $C_{I}$ on time indicates that the objectives, concerns, and guiding baselines of a system might change over time, as will be discussed in more detail in Chapter 4.

Unlike a "simple" system, a system of systems is composed of multiple component subsystems, each of which could be a "simple" system or a set of "simple" systems including technologies, policies, economies, and people, which are interconnected through often ambiguous physical, virtual, social, economic or other interdependencies. These sub-systems could be delineated based on physical, functional, organizational or other characteristics. Each of the sub-systems can be managerially and operationally independent and geographically and functionally heterogeneous, with their own set of objectives, decision makers, and stakeholders (Hall, 1989; Maier, 1998; Sage and Cuppan, 2001). In such a system, decisions are distributed and made independently in each sub-system, but they can nonetheless have an impact on the states and objectives of other interdependent sub-systems, as will be discussed in more detail in Chapter 4. As a result, any modeling or management approach that only takes into consideration a single sub-system of a system of systems is inadequate because it fails to capture the inherent sub-system interdependencies.

Figure 3 represents a model of a system of systems composed of two interdependent subsystems and it illustrates some of the challenges associated with modeling such systems. In these two sub-systems, decision makers are concerned with different perspectives of a system of systems (as outlined by the red and black colored sub-systems). 


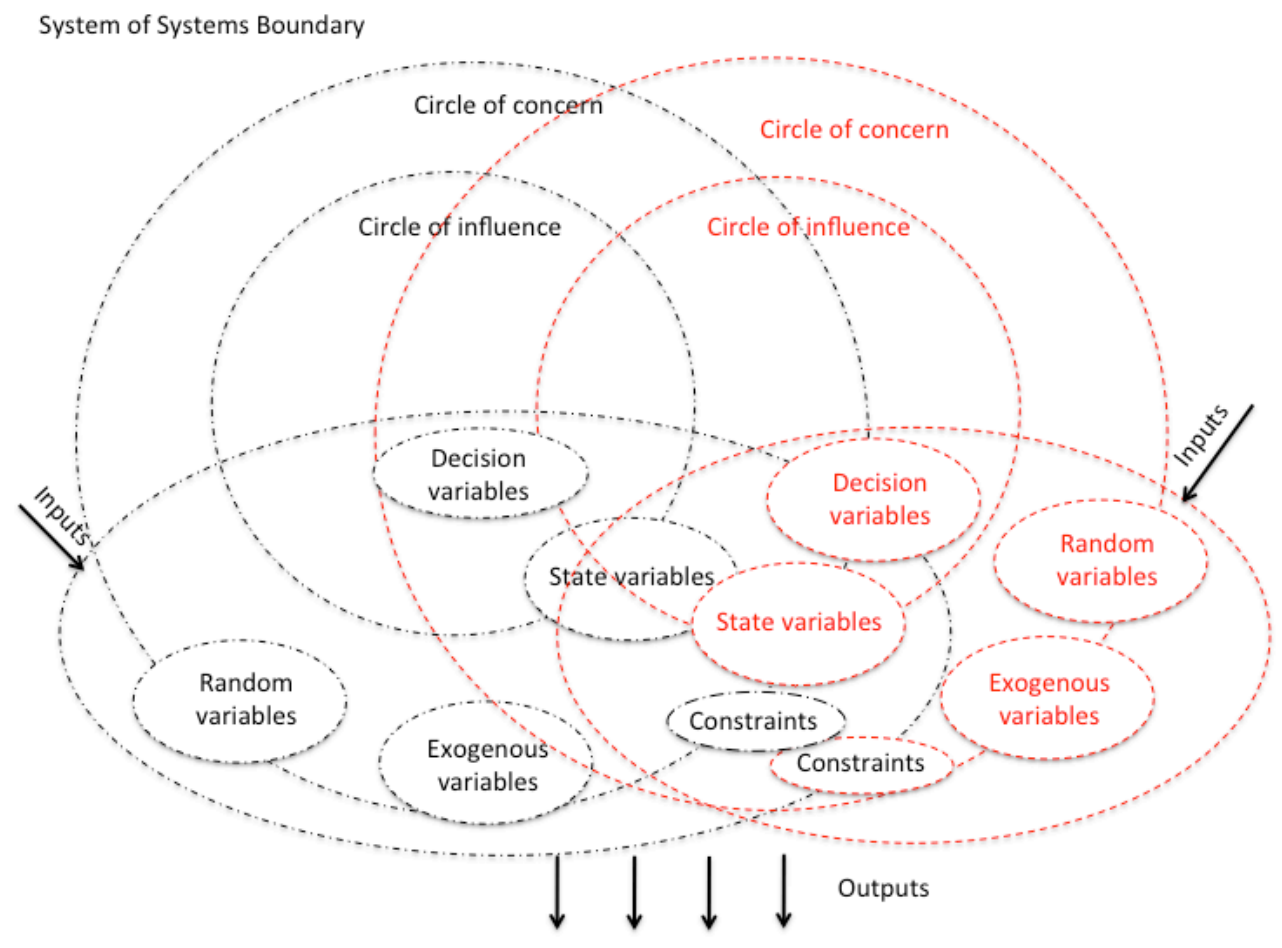

Figure 3: A model of a system of systems composed of two sub-systems (black and red lines indicate two interdependent sub-systems)

The two interdependent sub-systems are mathematically represented as follows:

\section{Sub-system 1:}

$\bar{s}^{1}(t)=f\left(\bar{u}^{1}(t), \bar{r}^{1}(t), \bar{e}^{1}(t), \bar{c}^{1}(t), \Phi_{1}\left(C_{I}^{2}(t)\right)\right)$

$\bar{y}^{1}(t)=f\left(\bar{u}^{1}(t), \bar{s}^{1}(t),\left\{u_{m}^{2}(t) \mid u_{m}^{2}(t) \in \bar{u}^{2}(t)\right\}\right)$

where

$$
\begin{aligned}
C_{C}^{1}(t)= & f\left(\bar{u}_{\text {decision }}^{1}(t), \bar{s}^{1}(t),\left\{r_{i}(t) \mid r_{i}(t) \in \bar{r}^{1}(t)\right\},\left\{e_{i}(t) \mid e_{i}(t) \in \bar{e}^{1}(t)\right\},\right. \\
& \left.\left\{c_{i}(t) \mid c_{i}(t) \in \bar{c}^{1}(t)\right\}\right) \\
C_{I}^{1}(t)= & f\left(\bar{u}_{\text {decision }}^{1}(t),\left\{s_{i}(t) \mid s_{i}(t) \in \bar{s}^{1}(t)\right\}, \Phi_{1}\left(C_{I}^{2}(t)\right)\right)
\end{aligned}
$$




\section{Sub-system 2:}

$$
\bar{s}^{2}(t)=f\left(\bar{u}^{2}(t), \bar{r}^{2}(t), \bar{e}^{2}(t), \bar{c}^{2}(t), \Phi_{2}\left(C_{I}^{1}(t)\right)\right)
$$

$$
\bar{y}^{2}(t)=f\left(\bar{u}^{2}(t), \bar{s}^{2}(t),\left\{u_{m}^{1}(t) \mid u_{m}^{1}(t) \in \bar{u}^{1}(t)\right\}\right)
$$

where

$$
\begin{aligned}
C_{C}^{2}(t)= & f\left(\bar{u}_{\text {decision }}^{2}(t), \bar{s}^{2}(t),\left\{r_{i}(t) \mid r_{i}(t) \in \bar{r}^{2}(t)\right\},\left\{e_{i}(t) \mid e_{i}(t) \in \bar{e}^{2}(t)\right\},\right. \\
& \left.\left\{c_{i}(t) \mid c_{i}(t) \in \bar{c}^{2}(t)\right\}\right) \\
C_{I}^{2}(t)= & f\left(\bar{u}_{\text {decision }}^{2}(t),\left\{s_{i}(t) \mid s_{i}(t) \in \bar{s}^{2}(t)\right\}, \Phi_{2}\left(C_{I}^{1}(t)\right)\right)
\end{aligned}
$$

such that,

$$
\left.\begin{array}{l}
C_{I}^{1} \cap C_{I}^{2} \neq \varnothing \\
C_{C}^{1} \cap C_{C}^{2} \neq \varnothing
\end{array}\right\} \Rightarrow \bar{s}^{1} \cap \bar{s}^{2}=\left\{s s_{l} ; l=1, \ldots\right\}
$$

In Eqs. 1-2 through 1-4, superscript $j=1,2$ indicates the two sub-systems, while the variables are described in the same manner as in Eq. 1-1. The overlap between the circles of concern and influence (Eq. 1-4) implies that decision makers share some concerns, objectives, and/or variables (otherwise the sub-systems would be independent and would thus not belong to the above system of systems). The two sub-systems have overlapping state spaces (Eq. 1-4), indicating that decisions made in one sub-system will have some effect on the states of the other sub-system since any decision invariably affects a subset of the system's state variables. Hence, the equation for the state space of a "simple" system (Eq. 1-1) is augmented by $\Phi_{1}\left(C_{I}^{2}(t)\right)$ and by $\Phi_{2}\left(C_{I}^{1}(t)\right)$ for sub-system 1 and 2, respectively (Eqs. 1-2 and 1-3), indicating that some of the decisions made in sub-system 1 (or 2) affect the states of sub-system 2 (or 1). In a larger system 
of systems with more than two sub-systems, overlapping among state spaces and circles of concern and influence may not exist among all sub-systems, but each sub-system shares at least one state variable with at least one other sub-system. In general, for $n$ sub-systems:

$$
\left.\begin{array}{l}
C_{I}^{i} \cap C_{I}^{j} \cap \ldots \neq \varnothing \quad(i, j \in n ; i \neq j) \\
C_{C}^{i} \cap C_{C}^{j} \cap \ldots \neq \varnothing \quad(i, j \in n ; i \neq j)
\end{array}\right\} \Rightarrow \bar{s}^{i} \cap \bar{s}^{j} \cap \ldots=\left\{s s_{l} ; l=1, \ldots\right\}
$$

In a system similar to this, it is no longer possible to easily identify the input-output relationships of a particular sub-system via state, random, exogenous and decision variables because outputs of sub-system 2 may, for example, be functions of some (possibly unknown) sub-set of variables from sub-system 1 as well, as indicated in Eqs. 1-2 and 1-3. Furthermore, those making decisions in one sub-system do not have absolute knowledge of how their decisions impact other interdependent sub-systems, and thus the outcomes of the overall system of systems. This implies that any modeling effort for systems of systems must account for the interdependencies among the sub-systems (i.e., $\Phi_{1}\left(C_{I}^{2}(t)\right)$ and $\Phi_{2}\left(C_{I}^{1}(t)\right)$ in the above equations).

Consider a surface transportation infrastructure, which is a system of systems composed of a network of roads, bridges and other physical assets, in addition to regional industries that use the network of assets for transporting commercial goods, non-commercial users who use the network for non-commercial purposes, and local, state, and federal decision and policy makers who make decisions about the network. In this system of systems, decision makers associated with a state Department of Transportation (DOT) are commonly concerned with maintaining adequate service levels of roads and bridges, while regional industries are concerned with the use of those same roads and bridges to most efficiently deliver intermediate supplies and finished goods. Consider an example in which regional manufacturing industries lobby at the federal level for higher legally allowable freight loads, which would enable them to carry larger loads and make fewer trips, and in the short run, reducing their freight transportation costs. A decision to allow increased loads would also affect decision makers in representative DOTs, because higher legally allowable freight loads would increase the average truck axle loading and the average 
stress exerted on bridges, which would in turn increase the rate of bridge deterioration and lower the bridge carrying capacity. In the absence of adequate and timely maintenance investments, in the long run the road and bridge surfaces would deteriorate to the point where speed or load postings might be required. Such postings may prevent freight carriers from utilizing certain bridges, thus forcing them to make detours, possibly resulting in unanticipated (emergent) additional costs to the regional industries. Thus, a policy originally intended to reduce the transportation costs of manufacturers might in fact result in higher overall costs for the regional industries. However, an explicit and common understanding of some shared variables, goals and constraints might prevent or reduce the extent of the unanticipated consequences stemming from the poor knowledge of the interdependencies.

\subsection{Contributions of the dissertation}

This dissertation builds on the premise that a system of systems cannot be adequately modeled through a single model, because a single model cannot account for the multiple perspectives and sub-systems that characterize such a system. Stakeholders and decision makers representing various sub-systems of a system of systems are managerially and operationally independent, and this creates a distributed and decentralized decision making environment in which outcomes of decentralized yet interdependent decisions made in one sub-system affect the states and objectives of the other interdependent sub-systems. As a result, any modeling or management approach that takes into consideration only a single sub-system of a system of systems is inadequate because it fails to capture the intricacies of sub-system interdependencies. Hence, there exists a need for an approach that enables one to harmonize multiple models representing different system perspectives.

To address this need, this dissertation presents a system of systems modeling framework that builds on the theory of Hierarchical Holographic Modeling (HHM) and Phantom System Models (PSM) intrinsic meta-modeling (Haimes, 1981, 2012), and permits one to model systems of systems through multiple harmonized sub-models (representing diverse stakeholders and subsystems). The need for such approaches is not new and was suggested in Hall's Metasystems Methodology in 1989: 
What is envisioned is a new synthesis, a unified, efficient systems methodology (SM): a multi-phase, multi-level, multi-paradigmatic creative problem-solving process for use by individuals, by small groups, by large multi-disciplinary teams, or by teams of teams. It satisfies human needs in seeking value truths by matching the properties of wanted systems, and their parts, to perform harmoniously with their full environments, over their entire life cycles. (Hall, 1989)

The specific contributions of this dissertation are threefold. First, this dissertation introduces an iterative, learn-as-you-go methodological process for the construction of inter-disciplinary models that represent different perspectives of a system of systems, and for their integration into a single meta-model through the use of shared state variables (discussed in more detail in Chapter 4). The dissertation presents some general modeling guidelines and insights, while not trivializing the complexity of the system of systems modeling process.

Second, from a theoretical perspective, this dissertation presents two contributions. First, a demonstration with a representative physical system of systems suggests that the knowledge of shared state variables transforms a black box system identification problem into a more informative grey-box system identification problem (discussed in more detail in Chapter 3). Second, this dissertation provides a theoretical discussion of how the meta-model can be used as an instrument for the coordination of decentralized, sub-systems' decisions. In Chapter 4 metamodeling coordination is defined as the iterative process of adjusting actions in interdependent sub-systems by manipulating decisions and constraints in response to measurable and/or observable changes in shared state variables in order to achieve objectives that are more acceptable to all system of systems stakeholders.

Finally, this dissertation demonstrates through a case study how the developed theory and methodology can be applied to the U.S. bridge infrastructure system of systems to examine the effects of untimely or insufficient bridge maintenance (discussed in more detail in Chapter 5). It is important to note that the purpose of this dissertation is neither to provide concrete maintenance solutions for a studied bridge, nor to provide detailed mathematical models representing different sub-systems of a bridge infrastructure, but, instead, to illustrate the potential value of modeling the bridge infrastructure via the PSM framework (to be presented in 
subsequent chapters), by coordinating multiple system- and stakeholder-level perspectives. Although in this dissertation the developed theory and methodology are applied to the bridge infrastructure, the contributions are sufficiently general to be easily transferable to other civil infrastructure systems of systems, thus addressing the need for approaches that are generic and transferable among different infrastructures (Jamshidi, 2009).

There are several theoretical and practical implications of the developed approach. First, it enables and motivates different stakeholders to identify, quantify, and explicitly model some common and conflicting interests and needs. Additionally, the interdisciplinary harmonization of knowledge and models enables decentralized decision makers that are in charge of different subsystems to adhere to satisficing policies that would be superior to competitive and uncoordinated

policies made at the sub-system level without the meta-modeling contribution. The case study can provide guidance for system of systems management by illustrating the extent to which meta-modeling can help with creating satisficing policies and solutions that are cognizant of and acceptable to all involved stakeholders. Finally, given the evolving nature of objectives, interest groups, organizational, political and budgetary baselines, and requirements associated with interdependent sub-systems, as well as the dynamics that characterize all systems, the metamodel coordination assumes an even broader role by enabling all stakeholders and decision makers to not only recognize the emergent future changes, but, more importantly, to plan for such changes through collaborative and foresighted efforts that would render beneficial outcomes for all parties.

\subsection{Overview of the dissertation}

Chapter 1 describes the current deficient state of the U.S. bridge infrastructure and it posits that the problem of bridge infrastructure management cannot be solved without understanding the scope of a larger problem, that of modeling large-scale systems of systems. Chapter 1 concludes by introducing the main objective of this dissertation - extension of the theory and methodology for system of systems modeling and management, and its application to bridge infrastructure management. 
Chapter 2 presents an overview of the relevant literature. The first section of this Chapter explores the literature that discusses some general issues associated with modeling systems of systems, and three major modeling paradigms that have been used to model systems of systems. The second section explores the literature on existing coordination approaches for systems of systems, including hierarchical decomposition approaches and multidisciplinary collaborative design optimization approaches. The third section provides an overview of the actual bridge management practices that are currently in place, and it contrasts those with the proposed system of systems approach to bridge infrastructure management. The fourth section provides an overview of the literature on modeling socio-economic effects of transportation infrastructure investments.

Chapter 3 demonstrates through a physical system of systems, the importance of accounting for sub-system interdependencies during the system identification process. It introduces the idea that sub-system interdependencies can be captured through essential state variables that are "shared" (common) between two or more sub-systems. By reverse engineering the known physical equations for a two component thermodynamic system of systems this chapter demonstrates that statistical models that account for sub-system interdependencies via shared state variables produce better fits to the underlying physical models based on first principles than those statistical models that do not account for sub-system interdependencies. Thus, the main contribution of this chapter is an initial demonstration of the validity of the claim that modeling sub-system interdependencies via shared state variables adds value to the system of systems modeling process by converting a purely black-box system identification model into a grey-box model.

Chapter 4 presents an iterative, learn-as-you-go methodological process for intrinsic metamodeling that builds on the theory of Hierarchical Holographic Modeling and Phantom System Models (PSM) intrinsic meta-modeling (Haimes, 1981, 2012), as well as a theoretical discussion about how the intrinsic meta-model can be used as an instrument for a collaborative coordination of decentralized sub-systems' decisions. The chapter addresses the need to model systems of systems from multiple perspectives, through multiple sub-models that are connected through extrinsic (input-output) and intrinsic (state variable) interdependencies. These sub-models are harmonized into a meta-model through shared state variables, which reflect shared aspects of the 
sub-systems, thus giving stakeholders a more holistic view of the entire system of systems. A major premise of this Chapter is that individual sub-systems may be managerially and operationally independent, resulting in a distributed and decentralized decision making environment in which outcomes of decentralized yet interdependent decisions made in one subsystem affect the states and objectives of the other interdependent sub-systems. This crossdisciplinary, decentralized, and distributed decision making environment calls for a collaborative management effort that can aid diverse and decentralized decision makers and stakeholders to assess the impact that their decisions have on the overall system of systems, and to collaboratively adjust their individual actions to achieve more satisficing global system of systems solutions. The collaborative and satisficing nature of this coordination process could increase the level of accountability that the general population has for bridge infrastructure, thus transferring risk ownership among all involved stakeholders.

Chapter 5 presents a case study in which the intrinsic meta-modeling process and coordination theory developed in Chapter 4 are applied to assess the effects of untimely and/or insufficient bridge maintenance of a strategically important bridge. This bridge is modeled as a system of systems, composed not only of engineered components, but also of commercial and non-commercial users, and federal, state, and local level decision and policy makers. The deployed methodology and theory enable and induce different stakeholders to identify, quantify, and explicitly model some common and conflicting interests and needs, with the ultimate goal of creating satisficing solutions and policies that are cognizant of and acceptable to a diverse array of involved stakeholders. The results of this case study illustrate the need for a more proactive and collaborative bridge infrastructure system of systems management process.

Chapter 6 goes beyond the intrinsic meta-modeling approach for system of systems modeling and management by suggesting that a new modeling paradigm, while needed, will not be sufficient in reforming the existing bridge infrastructure management practices on its own. Instead, what is needed are additional commitment devices that attempt to overcome the political, societal, cultural and psychological factors that frame the extent of possible solutions in infrastructure management. This chapter briefly describes some characteristics of the American political and policymaking process that tend to discourage strong regional planning and longterm proactive investments in infrastructure, as well as the psychological perception of risk and 
the resulting lack of public accountability. The chapter calls for a more continuous adoption of innovative funding mechanisms, like the Public-Private Partnerships, which can provide a stronger basis for proactive maintenance, and thus a mechanism for a better risk sharing among all stakeholders. The chapter concludes by suggesting that the intrinsic meta-modeling approach utilized in this dissertation provides a more transparent process through which all stakeholders might better understand how changes in the performance of infrastructures affect them directly, thus enabling them to make more informed trade-offs. Furthermore, the approach enables decision makers and stakeholders to better understand that by relegating infrastructure maintenance as a last minute effort most risks and costs are experienced by the users, and that inaction on part of any single stakeholder constitutes disaster in the long run.

Chapter 7 presents a brief conclusion and points to several potential future extensions of the developed work. 


\section{Chapter 2: Overview of Relevant Literature}

\subsection{Literature on modeling systems of systems}

The issues associated with modeling systems of systems are not new. In fact, in his seminal book Metasystems Methodology, Hall (1989) refers to these modeling issues by emphasizing the use of "a family of models at several levels to seek understanding of diverse aspects of a subject, and thus to comprehend the whole." He suggests that when dealing with large-scale systems an interdisciplinary approach to modeling systems is needed where the systems analyst acts as a broker and synthesizer of information. DeLaurentis and Callaway (2004) suggest that complex systems can be understood only through the concept of "horizontal thinking" in which knowledge is obtained from various disciplines. Bruijn and Herder (2009), in discussing sociotechnical systems, contemplate on how two modeling paradigms can be used in parallel, "thereby using the strengths of both worlds without damaging the strengths of either by full integration." Despite the pervasiveness of this topic in academic literature, the implementation of the multimodel, multi-disciplinary approach to real world systems of systems remains rather unsuccessful. In transportation infrastructure modeling, Aktan and Faust (2003) suggest, "there has not been any success in an integrated modeling of infrastructure hyper-systems inclusive of all of their critical engineered, human and natural elements." Furthermore, they suggest that the major challenge is "in adapting and integrating deterministic and stochastic, geometric and numerical, physics-based and "soft (data-or-knowledge based)", macroscopic or microscopic models developed by various disciplines for simulating infrastructure hyper-systems" (Aktan and Faust, 2003).

In recent years, much of the system of systems literature has focused on the definition and functional characteristics of such systems. Maier (1998), Sage and Cuppan (2001), Wells and Sage (2009), Boardman and Sauser (2006), DeLaurentis and Callaway (2004), DeLaurentis (2008), and Lewe et al. (2004) represent a miniscule portion of this literature, and they show that there does not exist a unified definition of systems of systems, nor does there exist a common approach for modeling them. In particular, Sage and Cuppan (2001) claim, "there is no 
universally accepted definition of these 'super systems.' What distinguishes a system of systems from other systems does not at this point have a definitive answer." Furthermore, DeLaurentis and Callaway (2004) claim that much confusion remains regarding what modeling approach should be used to best deal with transportation infrastructure systems of systems, and that "there is lack of systematic thinking at the basic level about how to address the challenges associated with SoS.” DeLaurentis (2008), Lewe et al. (2004), Parker (2010), and Aktan and Faust (2003) suggest that systems of systems problems require a new modeling paradigm that would account for the multiplicity of stakeholders, objectives, interdependencies and emergent outcomes.

Much of the literature dedicated to systems of systems discusses their "functional, organizational and structural nature; on the other hand, there has been comparatively less inquiry into the problem of modeling [systems of systems], and much of it has emerged within the last decade" (Haimes 2012). One of the approaches that has been an important research topic in the area of system of systems is the agent-based modeling and simulation approach (ABMS) (Axtell, 2000; Tesfatsion, 2002; Dooley and Corman, 2002; Farmer and Foley, 2009; Amin, 2000; Rinaldi et al., 2001; Rigole and Deconinck, 2006; Parunak et al., 1998). ABMS has gained momentum in the transportation community in the last four decades, where it is known as activity based travel demand analysis. NCHRP Synthesis 406 (2010) suggests that over the past few years several academic activity-based travel demand models have been developed that offer "something of interest beyond what has been implemented in practice." These include CEMDAP (University of Texas), FAMOS (University of South Florida), TASHA (University of Toronto), ALBATROSS (Eindhoven University of Technology, Netherlands). A most notable commercial application of an activity based travel demand model is certainly the Transportation Analysis and Simulation System (TRANSIMS) which was developed by the Los Alamos National Laboratory to 'meet the State DOTs' and MPOs' need for more accurate and more sensitive travel forecasts for transportation planning and emissions analysis" (FHWA - TRANSIMS). Unlike the traditional four step traffic demand models, TRANSIMS assumes that traffic is generated based on user demand for activities, thus the model first generates daily activities, and only then generates trips that satisfy those activities and assigns them on the transportation network. TRANSIMS generates a list of activities associated with each individual in a simulated household. These activities are based on demographic and activity surveys collected from real households in the study area (FHWA - TRANSIMS). TRANSIMS has been used to evaluate 
operational changes on highways and transit systems, and it has been tested for Dallas, Texas and Portland, Oregon (FHWA - TRANSIMS). While TRANSIMS shows great potential to model the emergent behavior of travelers, it requires extensive data collections and computational power, and has thus been sparsely used in real-world traffic planning practices. While representing an important contribution to the study of systems of systems, ABMS will not be of interest in this dissertation, which will focus instead on analytical approaches to modeling systems of systems.

Another important system of systems modeling paradigm is the system dynamics (SD) modeling approach. SD is a modeling method that was developed in the 1950s by Jay W. Forrester at the Massachusetts Institute of Technology (Forrester, 1961). Forrester "developed a philosophy leading to a systems viewpoint and a set of mathematical techniques for simulating complex, nonlinear, multiloop feedback systems" (Kim, 1998). Forrester defined SD as "the investigation of the information-feedback character of industrial systems and the use of models for the design of improved organizational form and guiding policy" (Forrester, 1961). Initial applications of SD were to general management problems, including inventory fluctuations, the changes in the labor force and falling market shares (Forrester, 1961). SD is grounded on the premise that "dynamic tendencies of a complex system arise from its causal and feedback structure" (Kim, 1998).

The SD modeling paradigm is in many respects similar to the general principles of Phantom System Models (PSM) meta-modeling that this dissertation builds upon. Both modeling paradigms assume that state variables are critical in modeling systems of systems and their interdependencies: "The overall system dynamics model can be simply generalized using the state variable, input variables, output variables, and the measures of the effectiveness of the system" (Kim, 1998). Both modeling paradigms recognize that decisions made in one subsystem affect state variables of other sub-systems, and thus affect the behavior of the overall system of systems. SD modeling paradigm provides a graphical interface through which causal relationships among the state variables and other essential variables of a system can be inferred and illustrated. The major elements of these causal mathematical models are feedback loops, flow stocks and time-delays. Stock and flow diagrams are used to generate the differential equations that govern the system evolution. Thus, the graphical feature of SD will be utilized in 
the PSM meta-modeling process to identify those variables (e.g., state, input, exogenous, random, decision, output variables) that are most essential for a system of systems under consideration, and that influence the evolution of other interdependent variables in the system.

SD models have been widely used for modeling long-term behavior of complex systems of systems due to their ability to identify causal chains, and model feedback functions and time lags (de la Barra 1989). Min et al. (2007) use the system dynamics approach to develop a model of the entire infrastructure system, thus displaying macro-level interdependencies among the different infrastructure sectors. Chasey et al. (1997) provide a brief overview of the SD model construction and indicate the main value of the SD modeling paradigm - identifying variables that are most important for the problem at hand and should thus be observed for the purpose of better infrastructure management. Hagani et al. (2003) suggest that SD models are powerful tools for evaluating the effects of policy implementation over a long time horizon.

In the transportation infrastructure domain, SD models have been used to analyze budget allocation policies for highway maintenance programs (Bjornsson et al., 2000; de la Garza et al., 1998), and study highway management practices (Chasey et al., 1997). Chasey et al. (1997) use SD to develop a framework that aids in "the understanding of the relationships between maintenance and construction expenditures on a highway system." In particular they use SD to identify and quantify a comprehensive level of service for highways that accounts for both the impact of deferred maintenance and highway obsolescence. Shen et al. (2005) use SD to study sustainable performance of construction projects. Hagani et al. (2003) construct an SD model composed of seven sub-models to assess the relationship between land use and transportation system performance. Ozbek et al. (2010) use SD to develop a "comprehensive framework that can measure the overall efficiency of road maintenance operations. This framework is designed to consider the effects of environmental (e.g., climate, location, etc.) and operational (e.g., traffic, load, etc.) factors on such overall efficiency." Kim (1998) utilizes SD to evaluate sustainable highway development. Wang et al. (2008) use SD to model urban transportation systems.

In this dissertation, the graphical interface of SD is utilized in the PSM meta-modeling process described in Chapter 4, and insights from Shen et al. (2005), Wang et al. (2008), Ozbek et al. (2010), Kim (1998), and Chasey et al. (1997, 2002) are used to identify essential states of a bridge system of systems in Chapter 5. 
In a recent effort to model systems of systems, Haimes introduces the Phantom System Models (PSM) framework (Haimes, 2007, 2008, 2012) that extends the basic theory and philosophy of Hierarchical Holographic Modeling (HHM) (Haimes, 1981) by "offering operational guidelines and principles on the basis of which to model systems of systems" (Haimes, 2012). The PSM framework emphasizes the need to use multiple models to represent the varied sub-models of a system of systems, and it relates the multi-perspective models at the meta-level through shared state variables.

The PSM meta-modeling assumes that "some specific commonalities, interdependencies, interconnectedness, or other relationships must exist between and among any two systems within any system of systems," and describes a meta-model as "a family of sub-models, each representing specific aspects of the subsystems for the purpose of gaining knowledge and understanding the multiple interdependencies among the sub-models, and thus allowing us to comprehend the system of systems as a whole" (Haimes, 2012). Each sub-model is built from a finite set of essential state variables, some of which are shared among interconnected subsystems. Haimes (2012) suggests that in a properly defined system of systems, any interconnected sub-system shares at least one (typically more) essential state variable(s) with at least one other sub-system (intrinsic interdependency), in addition to potentially shared inputs and outputs (extrinsic interdependencies). Haimes (2012) suggests that the PSM framework enables modelers to model inter- and intra-dependencies among the sub-systems of a complex system of systems by "exploiting vital knowledge and information embedded in the intrinsic and extrinsic common and uncommon state variables among the subsystems", however, he does not provide a specific methodological approach for deriving these sub-models and the meta-model, nor does he provide a quantifiable example that modeling through shared state variables in fact adds value to the process of modeling systems of systems.

This dissertation extends the PSM framework by: (i) demonstrating the validity of the claim that meta-modeling through shared state variables outperforms modeling efforts that do not account for sub-system interdependencies; (ii) building a methodological process for harmonizing sub-models into a meta-model via shared state variables, and for collaboratively coordinating decentralized decisions made in interdependent sub-systems; and (iii) applying the general principles of PSM to model a bridge infrastructure as a system of systems. The theory 
and methodology developed in this dissertation reflect the ideas voiced by Hall in 1989 and by DeLaurentis and Callaway in 2004 - that modeling of large-scale systems necessitates interdisciplinary approaches where the systems analyst acts as a broker and synthesizer of information, and more recently by Jamshidi (2009) who states that there exists a need to develop "generic theories and approaches such that lessons from one sector could easily be applied to other sectors." As will be discussed in subsequent chapters, existing methodologies, expert knowledge, databases, and models from various disciplines are utilized to construct the submodels and to coordinate them at the meta-level through shared state variables to address the problem of insufficient and untimely maintenance of a bridge infrastructure system of systems. The resulting cross-disciplinary, multi-model approach is general enough to be applicable to other infrastructure systems of systems, thus addressing the need for approaches that are generic and transferable between different infrastructures (Jamshidi, 2009).

\subsection{Literature on coordination approaches for systems of systems}

As was discussed in Chapter 1, the long-term wellbeing of the entire bridge infrastructure system of systems depends on the coordinated efforts of the individual sub-systems. While each level of control is fairly autonomous, to be a part of the entire system of systems, each sub-system must operate under some shared values and goals. Thus, long-term sustainability of the bridge infrastructure system of systems calls for collaborative coordination approaches. While the idea of sub-system coordination is not new, and has been explored in several research areas including hierarchical decomposition of large-scale systems and multidisciplinary collaborative design optimization, former coordination approaches have focused primarily on input-output interdependencies among sub-systems, whereas in this approach we focus primarily on interdependencies expressed through shared state variables.

In the area of hierarchical decomposition of large-scale systems, Lasdon and Schoeffler (1966) introduced a technique of decentralized control, which decomposes a large industrial system into smaller and simpler sub-systems, each of which is controlled separately. Individual sub-systems are coordinated by a higher-level controller that has an understanding of the individual and competing objectives of the sub-systems. In a similar manner, Haimes and 
Tarvainen (1980), Tarvainen and Haimes (1982), and Tarvainen et al. (1983) addressed the coordination process through hierarchical decomposition. In these studies authors developed methodologies in which each sub-problem is of lower dimensionality and containing fewer objectives than the original system of systems problem - after each sub-problem is solved, the solution of the overall problem is found by coordinating certain modeling parameters. Tarvainen and Haimes (1982), in dealing with static, deterministic, hierarchical systems with a single decision maker, defined coordination as a change in the coordination parameters (system's outputs) such that the system's underlying loss function decreases the fastest. In this single decision maker problem the authors developed both a feasible and non-feasible method for hierarchical decomposition, and they defined the coordination parameters as interaction variables, namely, outputs $\mathrm{y}_{\mathrm{i}}$ of individual subsystems. Tarvainen at al. (1983) derived feasible and nonfeasible decomposition methods for multi-objective dynamic problems involving multiple decision-makers.

More recently, Caballero et al. (2002) considered large-scale systems with multiple conflicting objectives, and proposed a two-level hierarchical method that decomposes the original problem into smaller multi-objective lower-level problems that are coordinated by an upper level that accounts for the relative importance of each sub-system. In particular, they introduced an iterative algorithm in which information is continuously exchanged between the upper coordination level and the lower sub-system level. In the above decomposition approaches, interdependencies are expressed in terms of inputs and outputs, and the input vector to each subsystem is expressed as a linear combination of the outputs of all sub-systems (see Figure 4). Coordination is performed by fixing the value of outputs of the sub-systems, and therefore of inputs into the sub-systems. This allows the above authors to decompose the overall problem into N smaller sub-problems, each of which can then be solved independently. Caballero et al. (2002) suggest that there are in fact many approaches to transform a constrained optimization problem into a multi-level optimization problem and that most of these approaches are in general a combination of feasible and non-feasible coordination. They suggest that in feasible coordination modifications are made only to the constraints of the sub-systems and that all intermediate values of coordination variables are feasible. In contrast, in non-feasible coordination modifications are made to the objective functions of the sub-systems and only the value reached at the end of the computation process is feasible. Caballero et al. (2002) critique the prior methods for sub- 
problem coordination, namely those proposed by Tarvainen (1986), and Li and Haimes (1987) as being difficult to implement in practical situations. The authors suggest that in Tarvainen (1986) a set of values for coordination variables must be fixed a priori and efficient solutions related to these fixed value may not, in fact, exist. With respect to Li and Haimes (1987), the authors suggest that the explicit parametric expressions for the set of efficient solutions may not exist, thus making this method difficult to implement in practical situations. They suggest: Li and Haimes (1987) “develop an enveloping coordination process, via a feasible decomposition theorem, for problems where the overall objective function is, by components, the sum of the objectives of the sub-systems. Nevertheless, this approach can only be used in practice when an analytic expression of the solutions of the sub-systems, as functions of the coordination variables, can be obtained. This is not frequent in practical cases" (Caballero et al. 2002).

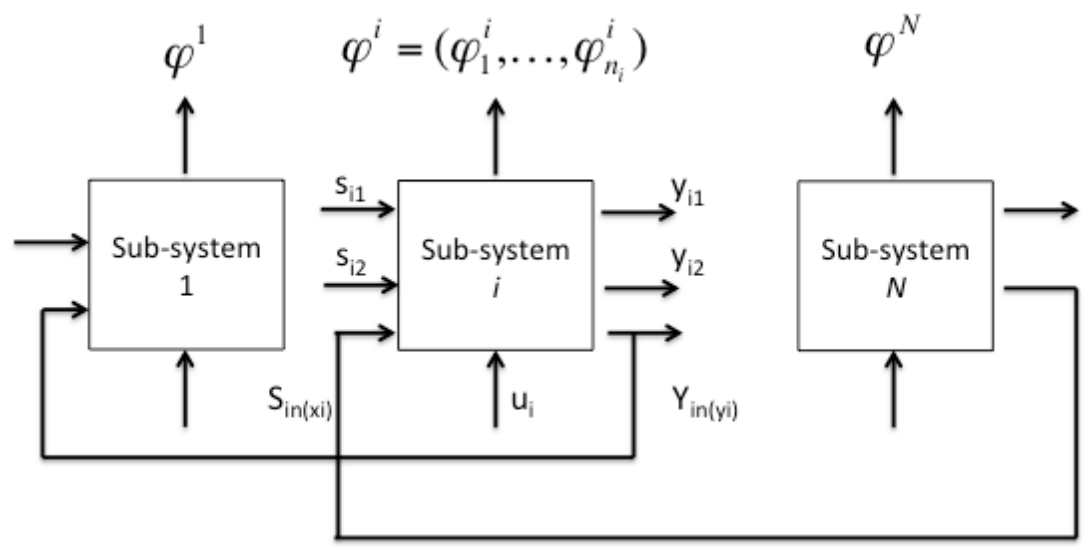

Figure 4: Hierarchical decomposition of a multi-objective large-scale system into several multi-objective ( $\left.\varphi^{i}\right)$ sub-systems. Interdependencies are expressed through shared inputs $\left(\mathrm{s}_{\mathrm{i}}\right)$ and outputs $\left(\mathrm{y}_{\mathrm{i}}\right)$, and coordination is performed by fixing the values of the outputs of the sub-systems. (Figure is adapted from Callabero et al., 2002)

In the area of multidisciplinary collaborative design optimization, each element (sub-system) of a complex system is designed and locally optimized by an independent discipline, which contributes its "respective competencies," and the overall system is the product of combinations of these independent designs and optimizations (Rabeau et al., 2007). Zadeh et al. (2009) suggest that collaborative optimization is "a bi-level optimization framework, with discipline specific 
optimization runs that are free to obtain local designs, and a system level optimizer to coordinate this process while minimizing the overall design objective." In such a process, couplings among the involved disciplines are characterized primarily through input-output dependencies (Rabeau et al., 2007), and the interdisciplinary coordination is characterized by the presence of a supervisory design level that supervises the global problem and manages the local design processes. Inalhan et al. (2002) "present a decentralized optimization method for solving the coordination problem of interconnected non-linear discrete dynamic systems with multiple decision makers." Their optimization framework embeds the inherent structure in which each decision maker has a mathematical model that captures only the local dynamics and the associated interconnected global constraints." The authors suggest, "when a centralized coordination scheme does not (or cannot) exist, the independent decision makers are forced to cooperate to achieve common or independent goals while operating under both local and interconnected constraints."

Unlike in the above works on hierarchical decomposition of large-scale systems and multidisciplinary collaborative design optimization, we premise that in addition to considering extrinsic (input-output) sub-system interdependencies, we must also consider the intrinsic interdependencies, which we express through shared state variables. Ultimately, the metamodeling coordination through shared state variables provides stakeholders with an opportunity to adjust their actions, based on measurable and/or observable changes in the shared states, to achieve satisficing solutions that may, over the planning horizon of interest, be more acceptable for the entire system of system, than solutions achieved without the meta-modeling coordination.

\subsection{Literature on existing bridge maintenance practices}

The theory and methodology developed in this dissertation are applied to a bridge infrastructure system of systems case study. In particular, the case study explores whether the PSM metamodeling approach provides additional insights into the problem of untimely and insufficient bridge maintenance, and if so, how these additional insights could be used to improve existing bridge maintenance practices. In order to make relevant modeling assumptions and draw conclusions from the case study, it is important to consider the nature and extent of existing 
bridge management practices. This section provides a brief overview of these practices.

The area of bridge management has evolved considerably over the last few decades, and many software tools have been developed to enable a more systematic approach to bridge management. Decision makers involved in bridge management can now rely on Bridge Management Systems (BMS) to aid them in the management of bridges through the various lifecycle stages: design, construction, operation, and maintenance. One of the most commonly used BMS, Pontis, was created to maintain up-to-date bridge inventories and inspection records, to create maintenance and rehabilitation plans, and to systematically and optimally allocate limited resources to maintenance projects (Robert et al., 2003). The Pontis software is available through the American Association of State Highway and Transportation Officials (AASHTO), and is used to help decision makers make more informed decisions regarding bridge maintenance, improvement and replacement projects. Specifically, the tool helps decision makers to answer questions regarding what kind of work is needed in the network of bridges and when it should be performed; which bridges should be maintained first given the limited resources; and what the possible impacts on the bridge network of delaying the recommended projects may be. In addition to providing guidance on project selection and resource allocation, Pontis also supports specific agency goal development, and project alternatives analysis. The maintenance recommendations created by Pontis are based primarily on element level condition data, deterioration models, and cost models (Robert et al., 2003).

While Pontis and other BMS have provided the much-needed framework for systematic evaluation of bridge maintenance needs, these tools are primarily concerned with technical and cost factors, and they generally ignore other considerations (e.g. socioeconomic factors, strategic or historical importance, environmental impacts, accessibility factors, development factors, safety, security, and service levels) that could influence the choice of optimal bridge maintenance plans over a longer planning horizon. In one word, they fail to provide a mechanism for a systemic and holistic management process.

Recently, in bridge management, and more generally in transportation infrastructure management, an increasing need has emerged to establish "a holistic investment decision-making framework ... which brings all social, environmental, economic, and political factors to bear in a logical and systematic way" (Zavadskas et al., 2008). A notable attempt to achieve a more 
holistic approach to bridge management has been developed in the area of multi-attribute decision-making (Zavadskas et al., 2008; Rashid et al., 2008; Chowdhury et al., 2002; Lounis et al., 2009; Patidar et al., 2007). While the principles of multi-attribute decision-making will not be utilized in the PSM meta-modeling framework, the general idea holds - in order to make more holistic, defensible, transparent and cost-sensible solutions different perspectives of a problem must be considered.

This dissertation will instead utilize information obtained from the National Cooperative Highway Research Program's (NCHRP) Guidebook for Assessing the Social and Economic Effects of Transportation Projects (2001) and a related document by Forkenbrock et al. (2001). These two documents provide an extensive overview of the current level of effort exerted by state Departments of Transportation (DOTs) and Metropolitan Planning Organizations (MPOs) to estimate the social and economic effects of transportation projects, and the models and tools available for assessing them.

As is evident from the reviewed literature, bridge management practices that are used by DOTs and MPOs are engineering- and cost- centric. In practice, no consistent effort is extended to try to understand how bridge maintenance decisions made by DOTs affect commercial and non-commercial bridge users, and similarly, how decisions made by commercial and noncommercial users affect the health of the bridge, and thus its maintenance needs. While models exist to evaluate more extensive socio-economic impacts caused by transportation infrastructure improvements, those models are only occasionally used in practice, and they are almost never used to assess the impacts of deterioration of existing transportation infrastructure.

\subsection{Literature on assessing the socio-economic impacts of transportation projects}

Much of the literature on social and economic impacts of transportation infrastructure investments comes from economic and regional sciences, which explore the economic impacts of transportation investments in new infrastructure projects (Jiang, 2001; Kim et al., 2004; Forkenbrock et al., 2001; Munnell, 1992; Aschauer, 1989; Bröcker et al., 2003; Weisbrod, 2008; Rephann and Isserman, 1994; Short and Kopp, 2005; Nadiri and Mamuenas, 1996; Weisbrod and Beckwith, 1992; Rietveld and Bruinsma, 1998; Oum et al., 1998; Babcock et al., 1997; Blum, 
1982). These studies suggest that investments in transportation infrastructure improvements have been shown to: expand the productive capacity of the region (Jiang, 2001; Aschauer, 1989); enable an easier transfer of resources between regions (Jiang, 2001; Kim et al. 2004); change the economic output of the region by changing the aggregate demand (Jiang, 2001); affect the regional distribution of wages and population (Kim et al., 2004); affect the GDP (Kim et al., 2004); affect the accessibility of supply chains (Weisbrod, 2008); and have a positive effect on export growth (Kim et al., 2004).

While literature abounds with articles studying the economic and regional development effects induced by new transportation infrastructure investments, little has been written about the socio-economic effects caused by insufficient or untimely investment in maintenance of existing transportation infrastructure. Ham et al. (2002) assess the economic impacts of transportation disruptions caused by a catastrophic earthquake, but their efforts are not focused solely on bridge failures. They consider the inter-industry relationships and regional commodity flows through the use of a multi-regional input-output model and a regional commodity flow model. Similarly, Cho et al. (1999) propose an integrated model that considers the economic losses due to effects of an earthquake on the transportation infrastructure and industrial capacity. Zhu et al. (2010) consider the I-35W Mississippi bridge collapse in Minnesota and deliver their findings on the behavioral effects caused by changes in bridge capacity.

National Cooperative Highway Research Program's (NCHRP) Guidebook for Assessing the Social and Economic Effects of Transportation Projects (NCHRP, 2001) provides a very extensive and well organized overview of the models, methods and databases that are currently available in practice for assessing the social and economic impacts stemming from transportation infrastructure investments, and this report will serve as a guide in selecting relevant models and databases for our analysis. In particular, the report reviews existing models and methods that can be used to assess changes in: travel time, vehicle operating costs, accessibility, economic development, community cohesion, safety, transportation choice, property values and distributive effects, all factors that relate to the travel patterns of both commercial and noncommercial bridge users. 


\section{Chapter 3: Modeling Systems of Systems Through Their Shared States: A Demonstration With a Thermodynamic System of Systems ${ }^{1}$}

\subsection{Main objectives}

Among the many challenges facing systems modelers is the need to determine the ways and means with which to enhance their knowledge about the system, discover its dynamic behavior, and identify the intra- and interdependencies among its sub-systems and learn its environment all while adhering to physical and other natural laws, basic principles in economics, social and organizational behavior, and considering other knowledge about the system. In many ways, modeling is the ultimate trial-and-error interplay between (i) theory and prior knowledge about a system and (ii) the learn-as-you-go inquisitive and exploratory processes of experimentation, measurement, and estimation.

Models are built to answer specific questions; they must be as simple as possible but as complex as required. This tradeoff is at the heart of model building, given that excessive complexity within a model is likely to impair its usefulness. Modeling a simple or a complex system to best represent its essence and functionality necessarily implies determining its properties, constructing the relationships among its inputs and outputs through its state variables and other variables and parameters, quantifying intra- and interdependencies within and among its various components and subsystems, and determining the appropriate model topology (structure) and parameters, i.e., performing system identification.

This chapter demonstrates through a representative physical system of systems, the importance of accounting for sub-system interdependencies during the system identification process. Furthermore it introduces the idea that sub-system interdependencies can be captured

\footnotetext{
${ }^{1}$ This chapter is based on a working paper by Haimes et al. (2013); results were obtained in collaboration with Ph.D. candidate Zhenyu Guo.
} 
through essential state variables that are "shared" (common) between two or more sub-systems. There is a fundamental difference between the complexity and challenges associated with modeling physical systems, which in their essence are controlled and driven by physical laws, and with modeling organizational and social systems. Yet, modeling systems of systems that are grounded on physical models, allows us to compare the results of our experimental system identification process to the physical models grounded on first principles. In contrast, larger and more complicated systems of systems, especially those composed of social and organizational sub-systems, often do not have existing analytical models that describe the system dynamics; we therefore describe the value of modeling via shared state variables for a physical system of systems, namely a two-component thermodynamic system.

By reverse engineering the known physical equations for a two component thermodynamic system of systems this chapter demonstrates that statistical models that account for sub-system interdependencies via shared state variables produce better fits to the underlying physical models based on first principles than those statistical models that do not account for sub-system interdependencies. We discuss why it is precisely shared state variables that add value to the system identification process, as opposed to simply considering shared input and outputs. We utilize metrics such as the Bayesian Information Criterion (BIC), Akaike Information Criterion (AIC), Adjusted Akaike Information Criterion (AICc), Mean Squared Error (MSE), and Adjusted $\mathrm{R}^{2}$ to compare the performance of models with and without shared state variables. Results of this analysis indicate that models with shared state variables outperform models without shared state variables, especially in cases dominated by sparse and noisy datasets.

It is important to emphasize that the major contribution of this Chapter is not the development of advanced models for the thermodynamic system of systems. Instead, the discussed example merely serves as an initial proof of concept that aims to show the validity of the claim that modeling sub-system interdependencies via shared state variables adds value to the system of systems modeling process. 


\subsection{Problem setup}

To demonstrate some issues associated with modeling systems of systems we perform an experiment with a simplistic physical system of systems, namely a thermodynamic system composed of two adjacent rooms (i.e., sub-systems), individually cooled by separate air conditioning $(\mathrm{AC})$ units. While each room is perfectly insulated from the outside environment, the walls separating the adjacent rooms are imperfectly insulated, thus allowing some transfer of heat between the rooms in question. Individuals residing in these rooms make independent decisions regarding the use of individual AC units; however, because of the heat transfer between the rooms, their independent decisions have unintended consequences on the temperature of the adjacent room. Hence, we describe this thermodynamic system as a system of systems that is composed of interdependent sub-systems (adjacent rooms) and of decentralized decision makers who make decisions independently, but whose decisions impact the state of the other sub-system (room).

\subsection{The modeling process}

Two students reside in adjacent dorm rooms. Each room is cooled by a separate AC unit, and each student is responsible for paying their own electricity bill. One of the students wishes to better control his utility expenses while achieving an acceptable temperature in his room. In order to best predict his utility expenses, this student needs a quantitative model describing the relationship between room temperature and the work performed by the AC unit. Assuming that the student has very limited knowledge about the thermodynamic system, his most practical approach is to develop a statistical model through system identification based on observed data. In order to do so, he identifies the system boundary to include only his room (Figure 5).

The student collects observations of measurable inputs (work exerted by his AC unit, $\mathrm{W}_{1}$ in Figure 5) and outputs (target inside temperature of his room, $\mathrm{T}_{\mathrm{L} 1}$ in Figure 5), and utilizes regression to obtain a mathematical representation between the two sets of measurements. He essentially utilizes a black-box system identification approach to identify the structure of the system, as his only available information are measurements of inputs and corresponding outputs 
(De Schutter, 2000; De Moor et al., 1999). Based on his input-output observations (derived in Appendix I), the student obtains the following representation of his one-room system:

$$
T_{L 1}=3.048 \times 10^{2}-7.633 \times 10^{-5} \times W_{1}+2.099 \times 10^{-10} \times W_{1}^{2}
$$

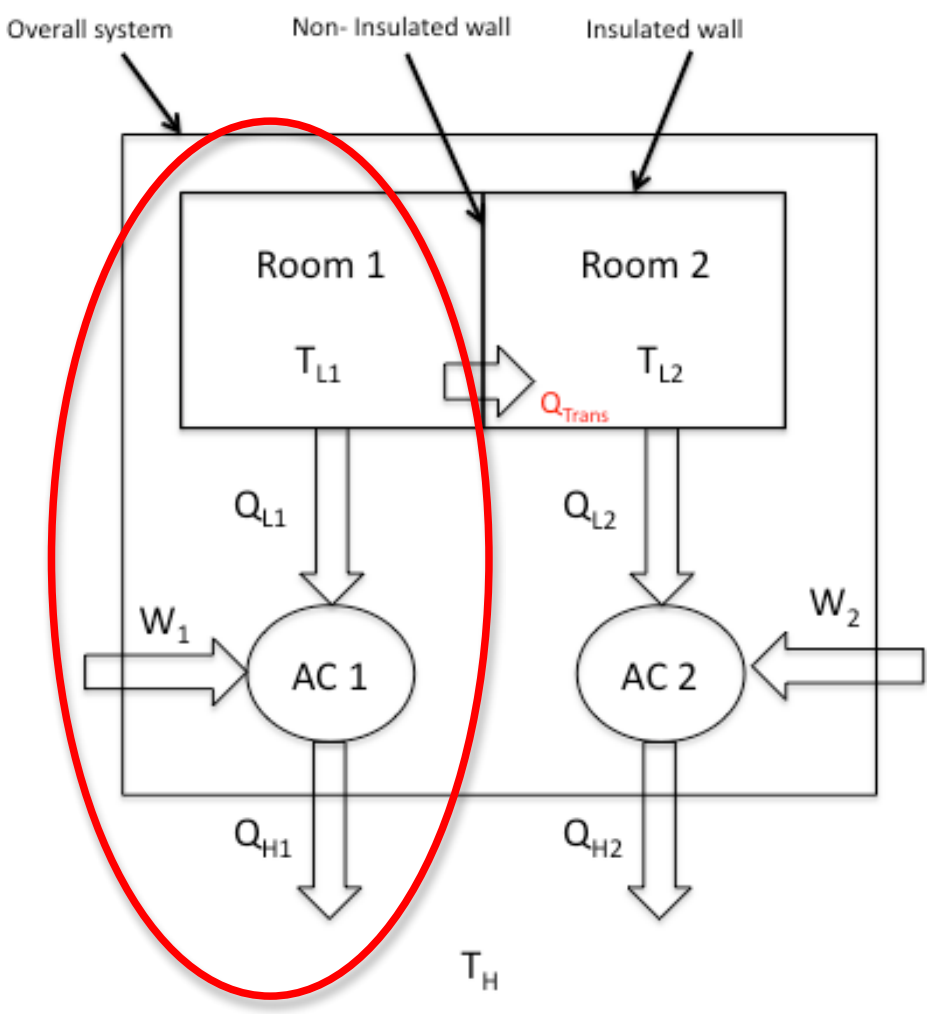

Figure 5: The two-room thermodynamic system of systems. Red oval outlines the initial boundary of concern for the student.

With respect to Figure 5, we let subscript $i=1,2$ represent the index of two sub-systems:

$t$ : Time

$T_{L i}(t)$ : Temperature of room $i$ observed at time $t$

$Q_{\text {Trans }}$ : Heat transferred between two rooms due to temperature difference

$Q_{L i}$ : Heat removed from room $i$ by air conditioner

$Q_{H i}$ : Heat output to environment from air conditioner $i$ 
$W_{i}(t)$ : Accumulated work to air conditioner $i$ observed at time $t$

$T_{H}$ : Environment temperature (constant)

The following month the student realizes that he cannot accurately predict his utility bill based on the black-box model expressed by Eq. 3-1. Upon further inspection, the student discovers that his room is not perfectly isolated from the adjacent room, causing some heat transfer between the two rooms. The student realizes that his initial understanding of the system boundary was wrong, and that his room is in fact a part of a larger system of systems, composed of his room and the adjacent room. He thus redefines his system boundary to include the adjacent room in addition to his own (see Figure 6).

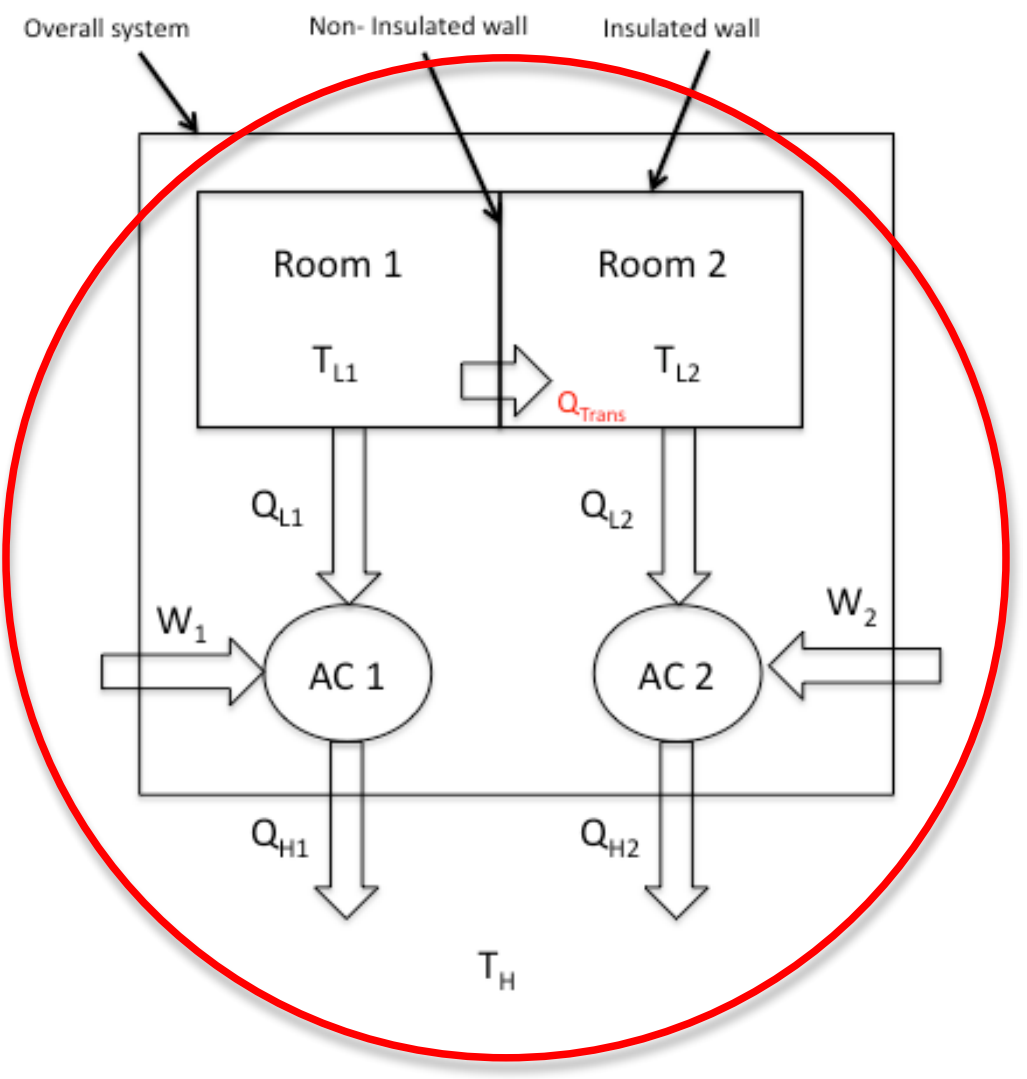

Figure 6: The two-room thermodynamic system of systems. Red oval outlines the redefined boundary of concern for the student. 
The student understands that the heat transfer represents interdependency between his room and the adjacent one, and that the amount of heat transfer depends upon his own actions (i.e., his decisions regarding the working of his $\mathrm{AC}$ unit), as well as the actions of his neighbor. The student is uncertain as to how to capture the interdependency between the two rooms, given that he cannot physically measure the heat exchange. The student hypothesizes that the interdependency between the two rooms might be best captured through shared state variables (state variables that are common to both sub-systems), because (i) "The state $\mathrm{x}\left(\mathrm{t}_{0}\right)$ of a system at time $t_{0}$ is the information at time $t_{0}$ that, together with the input $u(t)$, for $t \geq t_{0}$, uniquely determines the output $y(t)$ for all $t \geq t_{0}$." (Chen, 1999); (ii) the output of a system is directly a function of the state variables; and (iii) the level of state variables is directly altered by decision variables, the only variables he can fully control. He believes that by accounting for the subsystem interdependency, in addition to inputs and outputs of his own room, he might be able to construct a mathematical model that will more accurately predict his utility bills in the future. In other words, he believes that by utilizing the knowledge of the interdependency, he will be able to transform his black box modeling approach into a grey box modeling approach.

A grey-box modeling approach assumes that although one does not know the peculiarities of system dynamics, one has some insights into the system, which reduces the number of free parameters that have to be estimated through the process of system identification (see Figure 7). The figure on the left hand side of Figure 7 assumes that the student must have knowledge of the inputs and outputs of both rooms in order to develop a more representative model of his room's thermodynamic processes. However, the figure on the right hand side suggests that the knowledge of the shared state variable $s_{3}$ reduces the need to know the inputs and outputs associated with the adjacent room, thus reducing the number of parameters that need to be estimated through the process of system identification. Mathematically the grey-box model in Figure 7 can be expressed as in Eqs. 3-2 and 3-3, where $\mathrm{u}_{\mathrm{i}}(\mathrm{t})$ represents inputs and decisions, $\mathrm{s}_{\mathrm{i}}(\mathrm{t})$ represents system states, and $\mathrm{y}_{\mathrm{i}}(\mathrm{t})$ represents system outputs. 

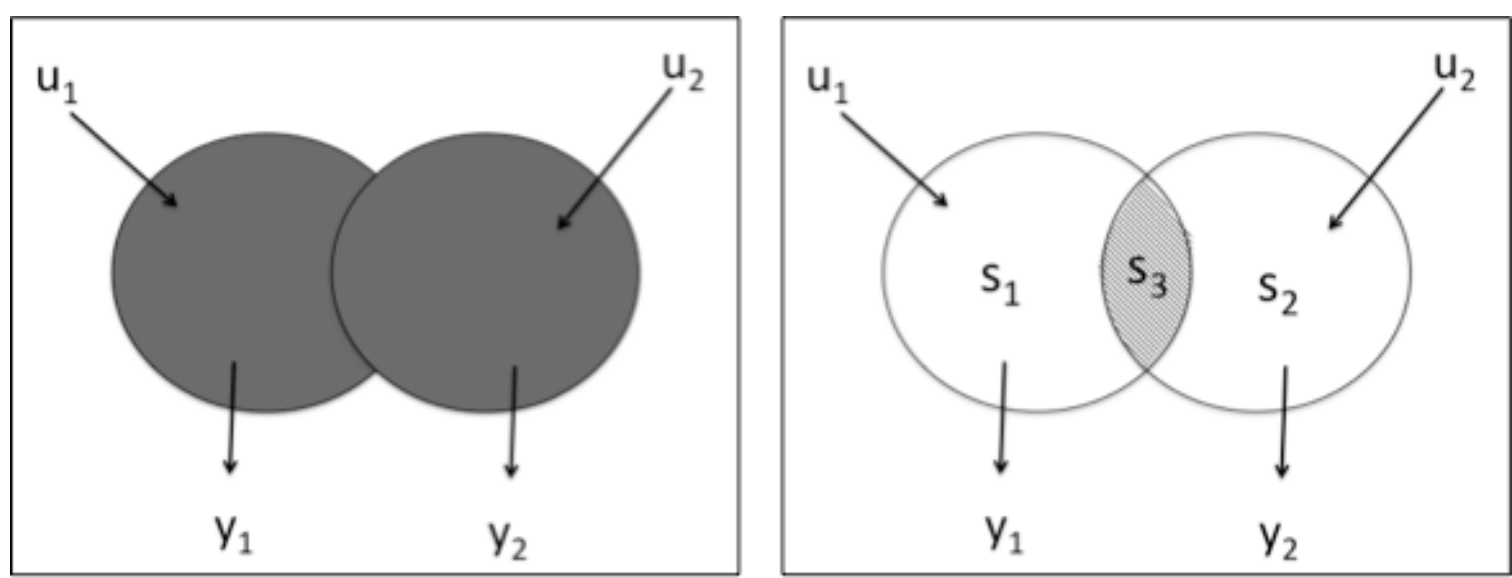

Figure 7: A black-box modeling approach is converted into a grey-box modeling approach through the introduction of a sub-system interdependency, expressed in form of a measurable or observable shared state variable.

Sub-system 1:

$s_{1}(t)=f\left(u_{1}(t), \Phi_{1}\left(s_{3}(t)\right)\right)$

$y_{1}(t)=f\left(u_{1}(t), s_{1}(t)\right)$

$\Phi_{1}\left(s_{3}(t)\right)=f\left(u_{1}(t), s_{1}(t), u_{2}(t), s_{2}(t)\right)$

Sub-system 2:

$s_{2}(t)=f\left(u_{2}(t), \Phi_{2}\left(s_{3}(t)\right)\right)$

$y_{2}(t)=f\left(u_{2}(t), s_{2}(t)\right)$

$\Phi_{2}\left(s_{3}(t)\right)=f\left(u_{1}(t), s_{1}(t), u_{2}(t), s_{2}(t)\right)$

The student hypothesizes about which state variable might be shared between the two rooms, in a way that would allow him to implicitly discern the actions of his neighbor with respect to her $\mathrm{AC}$, in addition to his own actions. In other words, the student realizes that in order to get a more 
holistic and representative view of the state of his own room, he must consider the problem from the perspective (context) of his neighbor as well. The student theorizes that the difference in the temperature between the two rooms may serve as a shared state variable to model the interdependency (heat transfer) between the two rooms, as heat transfer is not directly measurable. He utilizes a thermocouple to measure the temperature difference between the two rooms; based on this measurement he hopes to better capture his utility expenses without explicitly knowing what his neighbor is doing with her AC. The knowledge of the interdependency (i.e., the difference in temperature) should allow him to better model and predict his utility expenses.

The question then arises of why shared state variables might be of value to the student in this system identification process, in addition to shared inputs or shared decisions. While any variables that might be shared among sub-systems are important to consider in the modeling process, state variables determine the behavior of the system at any point in time, and they are in fact functions of these other variables at some point in time. Additionally, state variables are directly impacted by decision variables, thus shared state variables provide the student with an opportunity to measure the changes in heat transfer, and thus adjust his actions, without requiring him to have an explicit knowledge of the decisions that are made by his neighbor.

The student records measurements obtained through a thermocouple and utilizes those as shared state variables in his regression model, in addition to his measures of inputs and outputs. Through regression he obtains a new mathematical model of his system, shown in Eq. 3-4.

$$
\begin{aligned}
T_{L 1}= & 2.980 \times 10^{2}+1.485 \times 10^{-17} \times W_{1}-3.457 \times 10^{-23} \times W_{1}^{2}+ \\
& +3.5 \times\left(T_{L 1}-T_{L 2}\right)
\end{aligned}
$$

In order to compare the performance under actual conditions (simulated via a true physical model, derived in Appendix I), the student compares the performance of the two statistical models of his rooms (Eqs. 3-1 and 3-4) that he obtains from regression with the validation data. The student investigates the robustness of each model's performance by testing them under two 
different scenarios (derivation of the training and validation data points is described in Appendix I):

- Scenario 1: Student uses an uncontaminated full training dataset (101 training data points with no random noise)

- Scenario 2: Student uses a contaminated partial training dataset (20 training data points randomly selected from the full training dataset to which Gaussian random noise witz zero mean and standard deviation 0.01 was added)

To compare the predicted performance of the two models with the validation dataset (described in Appendix I), the student uses multiple criteria, such as the commonly used Mean Squared Error (MSE) and Coefficient of Determination. Since incorporating shared state variables increases the number of predictors, the student also utilizes criteria that penalize the addition of predictors. These criteria include Adjusted $\mathrm{R}^{2}$, Akaike Information Criterion (AIC), Bias Corrected Akaike Information Criterion (AICc), and Bayesian Information Criterion (BIC) (described in Appendix II).

In reference to Table 1, Model 1 (Eq. 3-1) assumes there are no interdependencies between the two rooms, and it relates the input of Room $1\left(\mathrm{~W}_{1}\right)$ to the output of Room $1\left(\mathrm{~T}_{\mathrm{L} 1}\right)$, while Model 2 (Eq. 3-4) assumes that interdependency exists between the two rooms, and that because of that the output of Room 1 depends on more than just the input of Room 1 . In Model 2, the student models this interdependency through a single shared state variable, namely, the difference in the inside temperatures of the two rooms (i.e., $\Delta T_{L}=T_{L 1}-T_{L 2}$ ) measured through a thermocouple.

MSE analysis for the two models indicates that in the case of a full and uncontaminated dataset, Model 2 outperforms Model 1 (see Table 1). Similarly, in the case of a partial and contaminated dataset, the shared state variable model significantly outperforms Model 1 (see Figure 8). All the other criteria indicate that Model 2 outperforms Model 1 under Scenario 1 (Model 2 has lower AIC, AICc, and BIC values). Table 1 and Figure 8 summarize the performance of the two models for Room 1. Note that Figure 8 represents a commonly used box and whisker plot which is a graphical representation of a five point summary, and in which the bold line in the middle of the box represents the median MSE of the 20 simulation runs obtained 
in Scenario 2, the vertical box lines represents the lower and upper quartiles of the MSE, and the lines extending from the box (whiskers) extend to the extreme minimum and maximum values of the MSE (indicated as points in the graph). We observe from Figure 8 that model 2 (model with shared state variable) produces results that have greater accuracy (minimal bias) and that appear to be more precise (i.e., show a smaller spread in estimated values indicated by the narrower height of the box).

\begin{tabular}{|l|c|c|}
\hline & Model 1 & Model 2 \\
\hline MSE & 126.4353 & $2.3997 \mathrm{e}-24$ \\
\hline R Squared & -29.9668 & 1 \\
\hline $\begin{array}{l}\text { Adjusted R } \\
\text { Squared }\end{array}$ & -30.6053 & 1 \\
\hline AIC & & \\
\hline AICc & 5.87973 & -53.3267 \\
\hline BIC & 5.90223 & -53.3025 \\
\hline
\end{tabular}

Table 1: Room 1 Models' Performance under Scenario 1

Through the identical process we obtain two models for the adjacent room, Room 2, and compare the performance of the two models under identical conditions (see Table 2).

Model 1:

$T_{L 2}=3.03 \times 10^{2}-3.525 \times 10^{-5} \times W_{2}+1.179 \times 10^{-11} \times W_{2}^{2}$

Model 2:

$T_{L 2}=2.98 \times 10^{2}+1.594 \times 10^{-18} \times W_{2}+2.313 \times 10^{-24} \times W_{2}^{2}+2.5 \times\left(T_{L 1}-T_{L 2}\right)$ 


\begin{tabular}{|l|c|c|}
\hline & Model 1 & Model 2 \\
\hline MSE & 0.1555 & $1.4766 \mathrm{e}-26$ \\
\hline R Squared & 0.9253 & 1 \\
\hline $\begin{array}{l}\text { Adjusted R } \\
\text { Squared }\end{array}$ & 0.9238 & 1 \\
\hline AIC & & \\
\hline AICc & -0.8211 & -58.4174 \\
\hline BIC & -0.7986 & -58.3932 \\
\hline
\end{tabular}

Table 2: Room 2 Models' Performance under Scenario 1

MSE Comparison for Room 1 Models

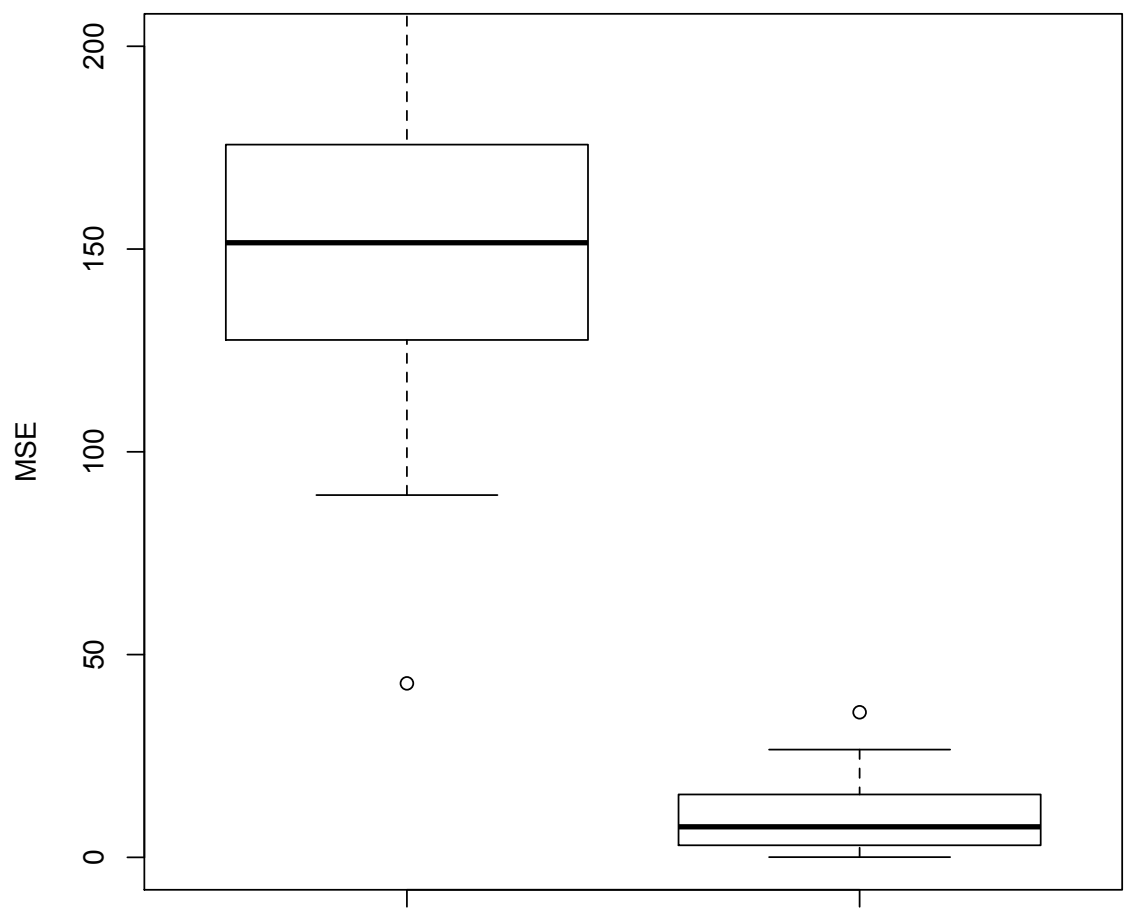

Left to Right: Model 1, 2

Figure 8: Comparison of Models' Performance for Room 1 under Scenario 2 


\subsection{Implications for larger and more complex systems of systems}

The preceding example demonstrated a fairly simple modeling process and suggested that the path to selecting the system boundary and the most essential variables is not straightforward, even in seemingly simple systems. In general, modelers usually begin the modeling process by identifying the questions they wish to answer and the corresponding system boundary of interest. Statistical models based on the observed data (or, occasionally, models based on first principles) are then developed for this system, and validated against relevant data. Often during this initial stage modelers determine that the models resulting from the initial system boundary specification are insufficient and do not account for all of the anticipated outcomes. As a result, they keep adjusting the system boundaries and relevant variables until they find the one that is more suitable. Thus, the modeling process is an iterative, learn-as-you-go progression that requires a continuous re-identification of the system's boundaries, stakeholders and the essential modeling variables. This process will be described in more detail in Chapter 4.

The process of identifying the essential shared state variable in this example was straightforward, primarily because the example was based on the knowledge of physical laws. However, this generally is not the case with larger and more complex systems of systems. Identifying essential state variables in a large system of systems is as much an art as it is a science. The set of essential state variables is very much dependent on the questions that we wish to answer with our models; thus, in order to identify the most essential state variables it is important to consider the multiple perspectives through which the system is being modeled.

The thermodynamic example demonstrated an important consideration in modeling systems of systems -- the presence of multiple sub-systems that are governed by independent and distributed decision makers. In systems of systems diverse stakeholders generally make decisions in a distributed and independent manner, without considering how their decisions might impact the other interdependent sub-systems. The thermodynamic example demonstrated that by identifying some states that are shared between interdependent sub-systems, we can better account for the impacts of decisions that are independently made in different sub-systems.

In Chapter 4 we describe in more general terms how the integration of the multiple modeling perspectives enables us to explore the similarities and differences of objectives, constraints and 
resources of interest in different sub-systems, and how the knowledge of essential shared state variables enables us to coordinate decisions made in independent sub-systems to achieve solutions that are more acceptable to all involved stakeholders. 


\section{Chapter 4: Methodological Process for Intrinsic Meta- Modeling and for Collaborative Coordination of Decentralized Decisions}

\subsection{Main objectives}

While much has been written over the last several decades about the meaning of systems of systems (for more details see Chapter 2), less has been said about modeling and managing such systems from an epistemological perspective (Hall, 1989; Haimes, 2012). Modeling of systems of systems, and their management, are difficult undertakings, encumbered by the number of interconnected sub-systems and the multitude of ambiguous interdependencies that govern the system dynamics. Thus, this dissertation posits that systems of systems cannot be adequately modeled through a single model, because a single model cannot account for the multiple perspectives that characterize a system of systems. As such, a multi-modeling approach is needed that takes into consideration the different system and stakeholder perspectives, and that provides a more holistic picture of the overall system of systems.

To address this need, this Chapter presents a system of systems modeling framework that builds on the theory of Hierarchical Holographic Modeling (HHM) and the Phantom System Models (PSM) intrinsic meta-modeling (Haimes, 1981, 2012). In particular, this Chapter presents an iterative, learn-as-you-go methodological process for: (i) the integration of crossdisciplinary models and related knowledge into a system of systems meta-model, and (ii) collaborative coordination of distributed and decentralized sub-systems' decisions. The implementation of this process to the bridge infrastructure system of systems management problem is described in Chapter 5.

A major premise of this Chapter is that stakeholders representing individual sub-systems may be managerially and operationally independent, resulting in a distributed and decentralized decision making environment in which outcomes of decentralized yet interdependent decisions made in one sub-system affect the states and objectives of the other interdependent sub-systems. 
This cross-disciplinary, decentralized, and distributed decision making environment calls for a collaborative management effort that can aid diverse and decentralized decision makers and stakeholders to assess the impact that their decisions have on the overall system of systems, and to collaboratively adjust their individual actions to achieve more satisficing global system of systems solutions. The collaborative and satisficing nature of this coordination process could increase the level of accountability that the general population has for bridge infrastructure, thus transferring risk ownership among all involved stakeholders.

The developed approach offers some general guidelines and insights, while not trivializing the complexity of the system of systems modeling and management process. Our approach is a creative process that recognizes the evolving and dynamic nature of the needs, requirements, and constraints associated with sub-systems and representative stakeholders, and that requires a continuous reassessment of the underlying models and projected solutions. It addresses critical needs identified in the literature, primarily the need for approaches that are generic and transferable between different systems (Jamshidi, 2009), and the need for "horizontal thinking" or cross-disciplinary approaches (DeLaurentis and Callaway, 2004). The developed process harmonizes multiple perspectives (allowing stakeholders to assess a system from multiple contexts), and in such a way ensures that the diverse and decentralized stakeholders avoid committing the "type-II error" (i.e., solving the wrong problem). Additionally, by requiring stakeholders to identify shared states through which they can relate their sub-systems to the entire system of systems, the meta-modeling process enables stakeholders to achieve global system of systems solutions that are holistically more acceptable over some planning horizon, while satisficing their own sub-system level objectives. In other words, the meta-modeling process motivates stakeholders to assume the responsibility of dual citizenship and subsidiarity (Handy, 1992; Sage and Cuppan, 2001).

Ultimately, the developed meta-modeling process represents the complexity of the decisionmaking processes associated with systems of systems, and it highlights the critical need for continued reassessment and cooperation, over the entire planning horizon, among the stakeholders representing different sub-systems. In the end, collaborative coordination provides a better opportunity for achieving solutions that are sustainable under diverse emergent forced 
changes, and are cognizant of the evolving needs, requirements and constraints of all the multiple and decentralized stakeholders and sub-systems.

\subsection{Dual citizenship, subsidiarity and satisficing}

Of particular interest in this dissertation are bridge infrastructure systems of systems, composed not only of the physical components, but also of the social, economic, political and natural environment within which they operate. As such, these systems of systems are not characterized by a structure in which one (or a subset) of the sub-systems has control over the other subsystems. Instead, these systems are characterized by distributed and diverse decision makers and stakeholders that work under a decentralized control scheme, and that might have opposing perspectives of a problem under consideration. The decision makers in individual sub-systems are fairly autonomous and in practice they typically make decisions as if they were completely independent from the other sub-systems, often unaware of the impact that their decisions might have on other sub-systems and on the overall system of systems. However, decisions made in individual sub-systems have an impact on other interdependent sub-systems, thus there exists a need for the decentralized and distributed decision makers to collaboratively coordinate their individual efforts to achieve more satisficing system of systems solutions that might be superior to competitive and uncoordinated policies made at the individual sub-system's level.

Satisficing is a term introduced by Herbert A. Simon and it refers to accepting suboptimal but adequate solutions (Simon, 1956). Ben-Haim (2012) suggests that "satisficing is more robust to uncertainty than optimizing," thus it is suited to the problem of coordinating multiple subsystems' decisions in a system of systems, due to the presence of many uncertainties. Satisficing the local and global objectives in a system of systems is particularly important from a broader public policy standpoint because "going after critical [global] requirements is usually a better bet for "survival," than going after what seems optimal" from an individual sub-system perspective (Ben-Haim, 2012).

The goal of satisficing is to achieve some pre-defined aspiration level - a decision maker is satisficed if the aspiration level is reached, and she is not satisficed if this level is not reached. Hence, the process of satisficing ends as soon as an acceptable level of the objective (i.e., a level 
at least as large as the aspiration level) is reached. While it may be possible to find subsequent satisficing solutions that dominate (in the Pareto-sense) the chosen solution, achieving this "better" satisficing solution might be very expensive in terms of time and resources, especially in situations in which multiple and conflicting stakeholders are involved in a collaborative coordination process.

To achieve more satisficing global system of systems solutions, decentralized and distributed decision makers and stakeholders must play two complementary roles - those of "citizens" of individual sub-systems and "citizens" of the entire system of systems, cognizant of the values, objectives and constraints of their individual sub-systems, as well as of the entire system of systems. In their discussion on the management of systems of systems and federations of systems (where a federation of systems represents a coalition of partners that have "decentralized power and authority and potentially differing perspectives of situations"), Sage and Cuppan (2001) refer to this principle as "dual citizenship." They suggest: "Every individual is a "citizen" in two communities, the local ... group/union, and the overall FOS [federation of systems] program at large," and this requirement implies the "willingness to ... avoid committing the "type-II" (wrong problem) errors ... This directly leads into the psycho-social notion of shared values." Sage and Cuppan (2001) additionally discuss the principle of "subsidiarity" which must be implemented in a federation of systems to ensure a " "type-II" accountability, or accountability for solving the correct problem." In the subsequent sections we will discuss how the harmonization of multiple and diverse modeling perspectives via shared state variables ensures that the involved stakeholders and decision makers are solving the right problem (i.e., avoiding the "type-II" errors) while serving as dual citizens, acting on behalf of their own sub-systems, as well as the entire system of systems. Given the evolving nature of objectives, interest groups, organizational, political and budgetary baselines, and requirements associated with interdependent sub-systems, as well as the dynamics that characterize all systems, the metamodel coordination assumes an even broader role by enabling all stakeholders and decision makers to not only recognize the emergent future changes, but, more importantly, to plan and continually reassess their efforts for such changes through collaborative and foresighted efforts that can render beneficial outcomes for all parties. 


\subsection{A need for collaborative coordination through shared state variables}

An important purpose of modeling large-scale systems of systems is to better understand and control the component sub-systems and the overall system of systems, and to coordinate the multiple and possibly competing objectives representing the needs and desires of the diverse stakeholders. As was discussed in Chapter 1, the long-term wellbeing of the entire bridge infrastructure system of systems depends on the coordinated efforts of the individual sub-systems. While each level of control is fairly autonomous, to be a part of the entire system of systems, each sub-system must operate under some shared values and goals. Thus, long-term sustainability of the bridge infrastructure system of systems calls for collaborative coordination approaches. While the idea of sub-system coordination is not new, and has been explored in several research areas including hierarchical decomposition of large-scale systems and multidisciplinary collaborative design optimization (discussed in Chapter 2), former coordination approaches have focused primarily on input-output interdependencies among sub-systems, whereas in this approach we focus primarily on interdependencies expressed through shared state variables.

In the theory of PSM intrinsic meta-modeling, shared state variables play a central role in defining interdependencies among sub-systems. A key premise of the PSM meta-modeling is that in a system of systems, there must exist at least one state variable that is shared among any two interdependent sub-systems. A lack of shared state variables indicates that the sub-systems are independent (i.e., they do not share any physical, virtual, social, economic, organizational or other properties, so decisions made in one sub-system do not in any way affect the other subsystems), and hence can be modeled completely independently. Shared state variables serve as an instrument through which diverse and decentralized stakeholders can explicitly model and assess the impact that their individual decisions have on other sub-systems, and on the system of systems as a whole. In other words, shared state variables enable stakeholders to understand their responsibilities as "dual citizens" representing specific sub-systems and the system of systems overall.

We define a shared state variable as that state variable which is explicitly or implicitly shared among interdependent sub-systems, such that it can be, over some period of time, directly impacted by decisions made by decentralized stakeholders representing different sub-systems. 
Note that shared state variables do not have to represent shared physical aspects of the subsystems, but can also represent shared virtual, social, economic, or other properties that explicitly or implicitly connect two or more modeling perspectives.

In Chapter 5, we explore the issue of insufficient and untimely maintenance of the bridge infrastructure from three perspectives - engineering, social, and economic. From the engineering perspective decision makers are concerned about the physical aspects of the bridge structure (e.g., amount of deterioration and its impact on condition ratings and load capacity), and they would like to ensure a sufficient condition rating for the bridge, as well as an adequate load capacity. From the social and economic perspectives, the problem of insufficient and untimely maintenance becomes an issue only when it starts affecting the ability of non-commercial and commercial travelers to safely cross the bridge during some period of time. Any maintenance decisions made by stakeholders in the engineering perspective will have an impact on the users of the bridge, and any decisions made by the users (e.g., decision to travel over the bridge, decision to carry heavy loads, decision to participate in infrastructure maintenance costs) will have an impact on the structural health of the infrastructure. Hence, one cannot evaluate the problem of bridge maintenance without considering it from multiple perspectives. In order to begin assessing the effects of bridge maintenance decisions, in Chapter 5 we introduce a shared state variable, namely the bridge traffic capacity. From the engineering perspective we relate the traditional bridge engineering metrics and the structural characteristics of a bridge to the traffic characteristics to obtain a metric that more adequately addresses the concerns of the interdependent sub-systems. Hence, from the engineering perspective bridge traffic capacity relates to the physical load characteristics of a bridge that determine the maximum number of all vehicles that can be carried over a bridge in a certain period of time. From the social perspective, it relates to the total number of non-commercial travelers that can safely cross over the bridge. From the economic perspective, it relates to the total number of commercial travelers (freight carriers) that can safely cross over the bridge.

The question then becomes of how the three different perspectives can be harmonized so that knowledge from one perspective can be shared with the others with the purpose of creating bridge maintenance policies that are cognizant of and responsive to emergent changes occurring in different sub-systems. As diverse stakeholders (representing the engineering, social, and 
economic perspectives) might have different views of what the "real problem" is, without a coordinated process they might all have different interpretations of what the actual value of the shared state variable is (see Eq. 4-1). Their interpretation depends on the limited context within which they operate, and on the time frame that is of interest to them (and that might differ from one perspective to the next).

Multi-perspective view of a shared state variable $m\left(\mathrm{ss}_{\mathrm{m}}\right)$ :

$$
s s_{m}(t)=\bigcup_{j=1 \ldots J}\left(s s_{m}^{1}(t), \ldots, s s_{m}^{J}(t)\right)
$$

such that

$$
\begin{aligned}
& s s_{m}^{1}(t)=f_{1}\left(\bar{u}^{1}(t), \bar{r}^{1}(t), \bar{e}^{1}(t), \bar{c}^{1}(t)\right) \\
& \vdots \\
& s s_{m}^{J}(t)=f_{J}\left(\bar{u}^{J}(t), \bar{r}^{J}(t), \bar{e}^{J}(t), \bar{c}^{J}(t)\right)
\end{aligned}
$$

$s s_{m}^{j}(t)$ represents a shared state variable $m$ evaluated from perspective $j$ at time $t, \bar{u}^{j}(t)$ represents a vector of inputs and decision variables relevant for the evaluation of a shared state variable $m$ from perspective $j$ at time $t$, and $\bar{r}^{j}(t), \bar{e}^{j}(t), \bar{c}^{j}(t)$, represent vectors of random variables, exogenous variables, and constraints relevant for the evaluation of a shared state variable $m$ from perspective $j$ at time $t$.

It is important to note that while the interpretation of the shared state variable might be different from different perspectives, ultimately, for an operational and sustainable system of systems, there must exist some range of values of a shared state variable that is acceptable from all perspectives. Through the intrinsic meta-modeling process, and the introduction of coupling functions that link shared and non-shared state variables in all sub-systems (described in more detail in Section 4.5), diverse and decentralized stakeholders can collaboratively combine their aggregate and cross-disciplinary knowledge to assess the impacts on the shared state variable from decisions and processes associated with different perspectives. A benefit of the meta-model coordination is that it combines different perspectives so that different views of the shared state 
can be integrated, and some globally acceptable values for the shared state can be achieved through a collaborative effort.

For the bridge case study described in Chapter 5, the three interpretations of the bridge traffic capacity must in the end equal some acceptable range of values in order for the bridge to be operational over a pre-specified period of time. Otherwise there exists a risk that the load exerted on the bridge might become greater than the actual physical capacity of the bridge. Therefore, for an operational and sustainable system, Eq. 4-2 must hold over the time period of interest.

$$
s s_{m}^{1}(t)=\ldots=s s_{m}^{J}(t) \quad \forall j \text { perspectives }
$$

While in reality the condition in Eq. 4-2 might not be true for all $t$, the goal of the metamodeling coordination, described in detail in Section 4.5, is to satisfy this condition over the planning horizon of interest, in order to minimize the risk of failing infrastructures and to ensure an adequate structural condition that will support the proper functioning of the socio-economic environment. In Section 4.4 we describe a modeling process that can be used to develop the meta-model and the supporting sub-models that represent the different perspectives of the problem, and that are connected through shared state variables.

\subsection{The iterative phases of intrinsic meta-modeling}

A thorough understanding of the true scope of the underlying problem, the time frame of interest, and the perspectives, goals, and values of the different stakeholders are necessary when modeling systems of systems. The consideration of the time frame is critically important, as changes over time may manifest themselves differently in different sub-systems. For example, in the surface transportation problem discussed in Chapter 1, the dynamics of physical changes to the bridge structure may be slow, and the physical impact on the load carrying capacity of the bridge caused by increased truck loads may not be visible until many years after the fact, while the economic benefits of increasing truck loads are evident almost instantly. Hence, the scale and time of impact of certain decisions need to be considered over an appropriate planning horizon. 
In addition to the time frame, a thorough understanding of the respective values, needs, goals, and constraints of involved stakeholders, enables decision makers to hypothesize about the future in a systemic way, by building multi-perspective risk scenarios for the planning period of interest. In order to consider these factors, varied stakeholders need to utilize a collaborative approach that provides them with an understanding of how sub-system specific details fit within a larger system of systems over context-specific time frames. Therefore, they need a top-down modeling process that takes into account the importance of the time frame, and the changing needs, constraints, and requirements of all involved stakeholders. Figure 9 illustrates such a modeling process, where the meta-model represents a collection of different modeling perspectives (quantitative sub-models and meta-model are presented for a specific problem in Chapter 5). Each modeling perspective views the system and the underlying problem from the perspective (context) of different stakeholders, and each is composed of supporting sub-models (i.e., mathematical representations of the sub-system under study).

As Figure 9 suggests, and as will be discussed in more detail in subsequent sections, the submodels are harmonized at the meta-level through shared state variables, and through this process individual stakeholders are able to better visualize the need to harmonize the sometimes conflicting objectives into satisficing solutions acceptable to all stakeholders. 


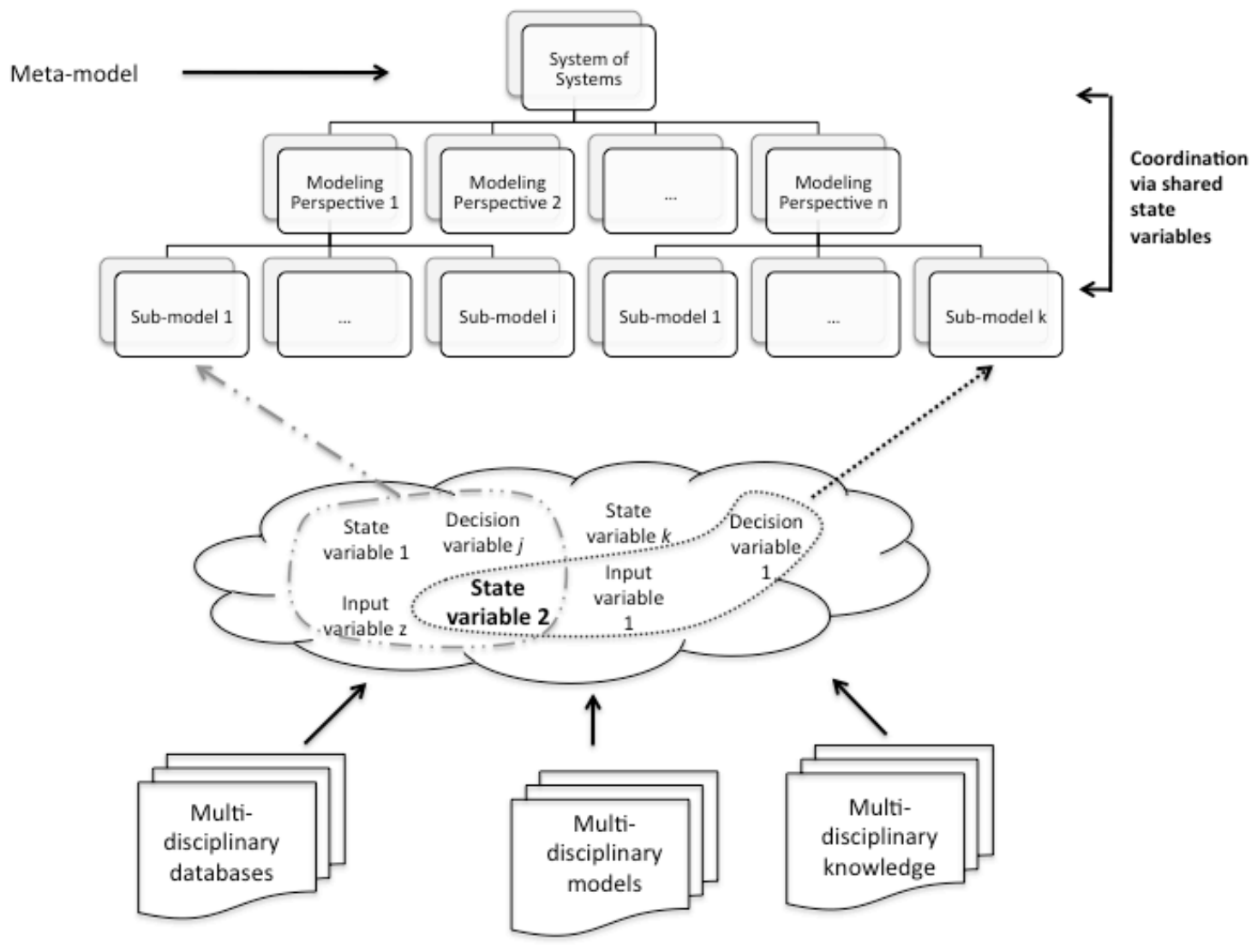

Figure 9: A top-down view of the meta-modeling process

\subsubsection{Identifying essential modeling perspectives and questions}

In the modeling process depicted in Figure 9, selecting a finite and manageable set of modeling perspectives mimics the HHM process cited in Section 4.1. Initially a collaborative and crossdisciplinary group of experts, decision makers, and other stakeholders, aided by modelers, should identify as many modeling perspectives (i.e., head topics in HHM) as possible, including potentially overlapping ones. This is done to gain a broader understanding of the overall problem and to minimize the possibility of committing type-II errors (solving the wrong problem). Through the process of negotiation and information exchange, these perspectives can then be grouped into a smaller set of more general perspectives, guided by some common concerns or 
constraints. This is done to simplify the modeling process, as models should be as complex as required, but as simple as possible. For example, some initial modeling perspectives in the surface transportation example discussed in Chapter 1 may include concerns with changes in: travel time for commercial and non-commercial travelers, vehicle maintenance costs, real estate values, and economic development patterns due to changes in the usage of bridges. At this stage, these perspectives could be grouped into three general modeling perspectives, namely, social, engineering, and economic perspectives, which are used to envision an array of future risk scenarios. A prioritized (negotiated) list of risk scenarios is then used to identify the most relevant questions that stakeholders representing different modeling perspectives would like to answer. The following steps require that the supporting sub-models to answer these questions be built or identified in the existing literature.

\subsubsection{Identifying representative sub-models, shared and non-shared variables}

Figure 9 suggests that modeling perspectives are collections of supporting sub-models, which are mathematical representations of the sub-systems under study (e.g., in the surface transportation problem discussed in Chapter 1, these could include various engineering sub-models for different types of bridges and pavements, economic sub-models for the movement of commercial goods between different regional industry centers, etc.) As was already mentioned, these sub-models must be able to answer the specific questions that were identified in the prior phase. Each submodel is connected to at least one other sub-model through some shared state variable(s).

The collaborative and cross-disciplinary nature of this modeling process comes into full play in this phase, requiring a thorough examination of existing cross-disciplinary (multi-perspective) models, databases and knowledge. In certain cases models will already exist that can be used to answer specific modeling questions, but often, new models will have to be built to more adequately address the stakeholders' concerns. In those instances, existing cross-disciplinary knowledge, models, and databases can be used to identify the large number of variables that might be needed to build sub-models that answer particular modeling questions. Hence, at this stage stakeholders must identify all variables that even remotely address their modeling questions. These variables are filtered down to the most essential variables at the next stage, but 
at this point it is important to be as encompassing as possible so as to not unintentionally leave out of consideration any important factors.

A collaborative consideration of the multi-disciplinary factors that have already been identified in the existing literature is a first step in enabling stakeholders to visualize the interdependencies that their sub-systems might share. At this stage it is not necessary to fully understand the extent and dynamics of those interdependencies, although it is important to identify some common factors that might be of interest from different modeling perspectives. These shared factors might include shared inputs or outputs, random and exogenous variables, shared decisions, or shared state variables. Shared state variables are illustrated in Figure 9, where sub-models 1 and $k$ are both dependent on state variable 2 hence this variable represents a shared state variable. In the surface transportation system of systems, the monetary value of travel time might be a factor that is important to stakeholders from both the social and economic modeling perspectives, while the physical roadway capacity might initially be of direct importance only to the stakeholders from the engineering perspective. However, a collaborative examination of these factors suggests that the two factors are related and the physical roadway capacity ought to be of importance from both the economic and social perspectives. Therefore, at this stage it is useful for modelers to "harvest" from existing knowledge, models, and databases the relevant state, input, output, and decision variables that can be utilized in the following stages to construct mathematical relationships among the shared and non-shared variables.

\subsubsection{Identifying essential modeling variables}

In a previous phase, discussed in Section 4.4.1 above, stakeholders posed questions that they would like to be able to answer with specific sub-models. For the bridge maintenance problem discussed in Chapter 5 these questions might include: "What is the impact on the bridge load capacity of a particular maintenance plan?"; "Are there financial and other long-term benefits from proactive bridge maintenance?"; "What are the immediate and long-term impacts on the ability of commercial and non-commercial travelers to utilize the bridge of interest?"; "Are there innovative funding sources that could be implemented to enable a more proactive bridge maintenance scheme?". Because it would be computationally inefficient and mathematically 
intractable to consider a very large set of variables in a model, at this stage stakeholders, with assistance from modelers, must identify those variables that are absolutely essential in answering the modeling questions associated with each modeling perspective. This process is as much art as science, and the mantra that guides this step is that models should be as simple as possible and as complex as required to answer the questions at hand (Haimes, 2009). This process can be facilitated through the use of influence diagrams, system dynamics diagrams, and expert knowledge or through a statistical evaluation of the significance of individual variables.

System dynamics and influence diagrams can, for example, be used to relate different time frames and different contexts, and to concurrently visualize variables that should be considered to assess objectives of different sub-systems, and to identify overlaps between those variables, thus between different modeling perspectives (see Figure 10). Having a holistic view of the relevant modeling variables a priori can help stakeholders to determine which variables are most essential to consider given the changing and diverse perspectives.

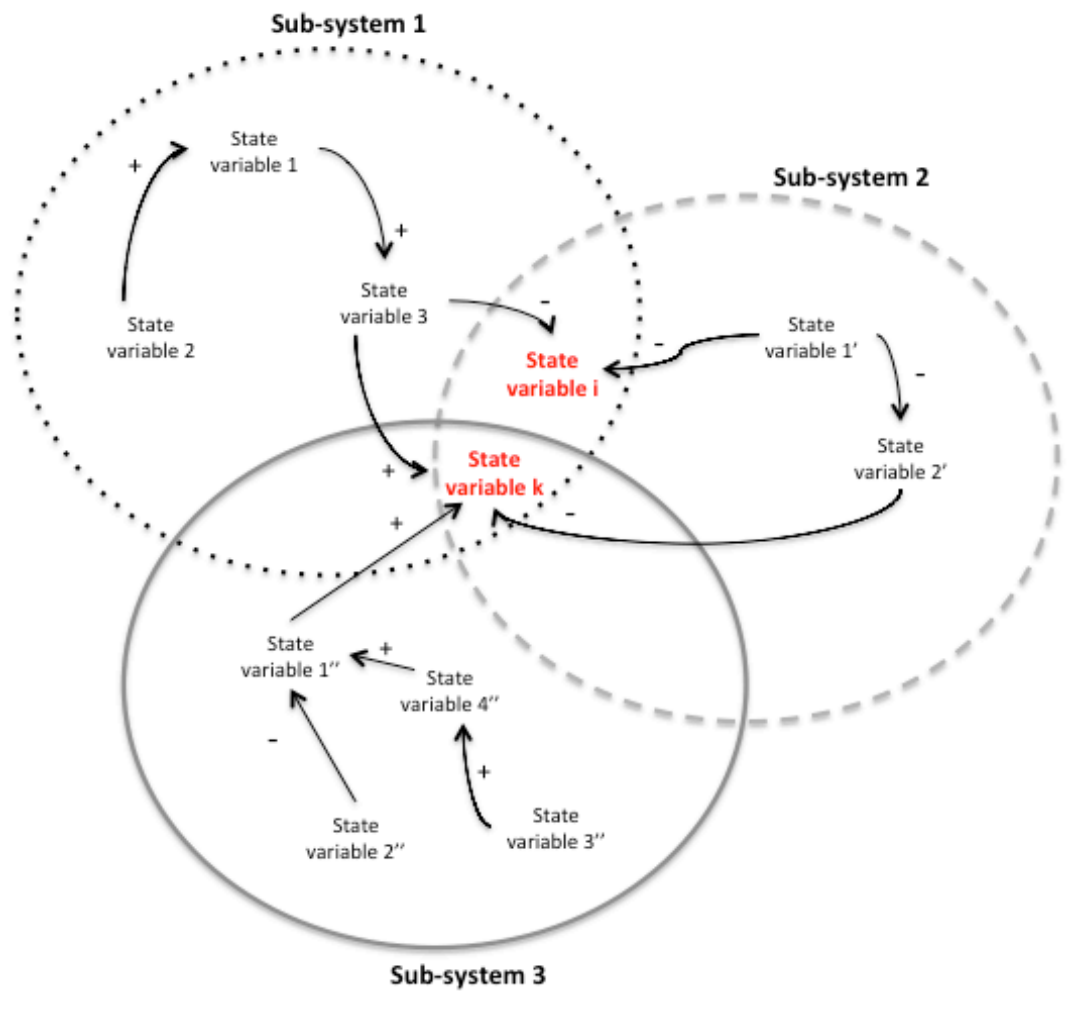

Figure 10: Example of how a system dynamics diagram can be utilized to identify essential shared and nonshared variables for different sub-models. Shared variables are in red. 


\subsubsection{System identification}

As was already mentioned, occasionally modelers will identify existing models in the literature that can be used to adequately answer some of the stakeholders' modeling questions. More frequently, however, they will have to construct new models that specifically address the questions at hand (this was discussed in Chapter 3, and will be discussed further in Chapter 5). In this case, after the identification of essential shared and non-shared variables, modelers will have to explicitly determine the mathematical relationships among those essential variables. The process of determining the topology of mathematical models, and the parameters associated with essential variables, is called system identification. The goal of the system identification process is to find a mathematical representation of the system that is simple enough, but is as complex as needed. For example, consider a linear time-invariant (LTI) system defined by the following set of equations:

$$
\begin{aligned}
& \bar{s}(k+1)=A \bar{s}(k)+B \bar{u}(k)+\bar{\omega}(k) \quad k=0, \ldots, T-1 \\
& \bar{y}(k)=C \bar{s}(k)+D \bar{u}(k)+\bar{v}(k)
\end{aligned}
$$

In this LTI system representation, the process of system identification requires determining the order $n$ of the unknown system (where the order denotes the number of elements in the state vector $\bar{s}(k)$ ), and the system matrices $\mathrm{A}, \mathrm{B}, \mathrm{C}$, and $\mathrm{D}$, given a finite number of possibly noisy measurements of inputs and decisions, $\bar{u}(k)$, and outputs $\bar{y}(k)$. As was mentioned in Chapter 3 , system identification is divided into three main approaches (De Schutter, 2000; Ljung, 2010). The "white-box" approach assumes that one can derive the mathematical representation of a system by determining the fundamental physical laws that govern the system dynamics. This approach is only used for the simplest systems. The "grey-box" approach assumes that one does not know the peculiarities of system dynamics, but that one has some insight into the system, which reduces the number of free parameters that have to be estimated through the process of system identification. The "black-box" approach is used to identify the structure of the systems for which one can only collect measurements of the inputs and the corresponding outputs, and 
then use this data to find a mathematical representation that best describes the measured data. Two different classes of methods are generally used to create representative "black-box" models, namely the prediction error method and the subspace method. Detailed information about the differences between the two methods is given in De Schutter (2000) and De Moor et al. (1999).

As was demonstrated in Chapter 3 through an example of a physical system of systems, at this stage of the system of systems modeling process, modelers must determine whether any of the sub-systems can be described by white-box models, i.e., represented through models based on first principles. If it is possible to model any of the sub-systems in this way, these sub-systems should be modeled first, and then the knowledge of the shared state variables should be utilized to more easily model the remaining interdependent sub-systems. If it is not possible to model any of the sub-systems through white-box approaches, and in most cases it will not be possible to do so, modelers must resort to grey- or black-box methods.

In Chapter 5 we will utilize the LTI representation to represent the different modeling perspectives of the bridge infrastructure system of systems. While systems of systems are generally not linear or time-invariant, the LTI topology has been used in the past to represent many systems, including complex systems (De Schutter, 2000; Mäkilä and Partington, 2003; Mäkilä and Partington, 2004; Mäkilä, 2004; Schoukens et al., 2003). There are several advantages of utilizing an LTI topology to demonstrate the usefulness of intrinsic meta-modeling. Advantages include: closed-form solutions for LTI systems; possible expansion of topology for non-linear systems; time-invariance implies constant parameters; possible expansion of topology for time-varying systems; focus on the time-domain and the resulting ability to compare dynamic tradeoffs over time.

\subsubsection{Continuous re-evaluation}

As was already indicated, the system of systems modeling process is not an exact science, but a creative, evolving, learn-as-you-go process. If the system of systems' boundary changes, for example, by a change in the number of modeling perspectives under consideration, or by a change in the essential variables, the modeling process must be repeated. If new information is discovered about the system of systems or any particular sub-system, the modeling process 
should be re-evaluated to explore how the new information affects each modeling perspective. Given the reality of the constantly shifting stakeholders' values, objectives, requirements, and constraints, we cannot always assume that the developed models will accurately capture the subsystems or a system of systems over the entire long planning horizon. Thus, we emphasize the need for a continuous (within practical limitations) re-evaluation of the modeling process over the planning horizon of interest. The iterative nature of the process described in Sections 4.4.1 4.4.4 is illustrated in Figure 11.

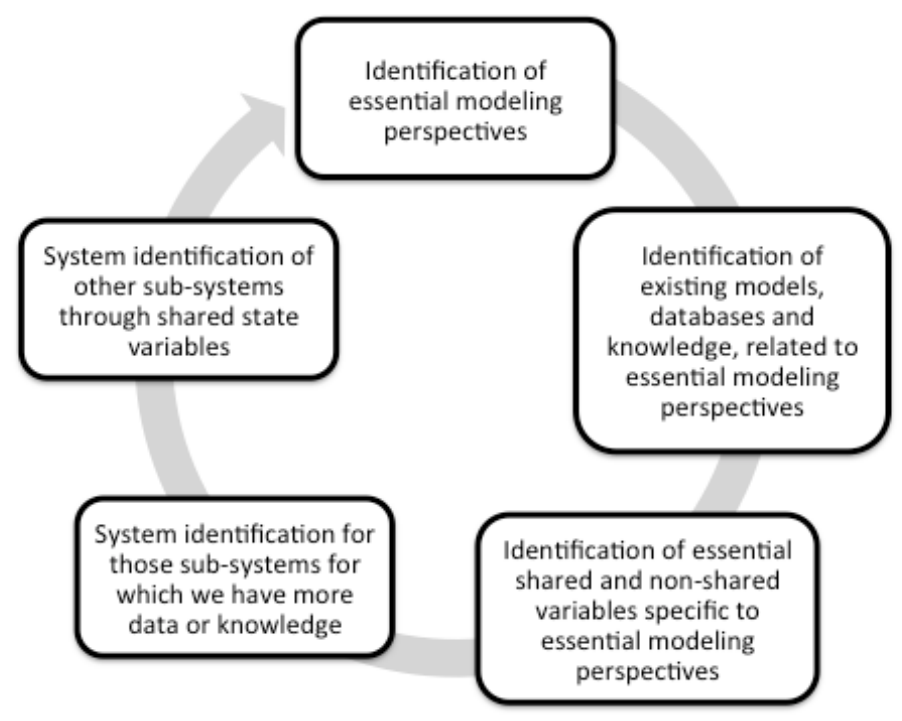

Figure 11: Overview of the iterative intrinsic meta-modeling process

\subsection{Intrinsic meta-modeling coordination}

Over the last several decades, many definitions of coordination have been proposed, some of which are reviewed here. In 1989 the National Science Foundation (NSF, 1989) defined coordination in several ways:

- "The operation of complex systems made up of components"

- "The emergent behavior of collections of individuals whose actions are based on complex decision processes" 
- "Information processing within a system of communicating entities with distinct information states"

- "The joint efforts of independent communicating actors towards mutually defined goals"

Singh (1992) defined coordination as the "integration and harmonious adjustment of individual work efforts towards the accomplishment of a larger goal”, while Malone (1988) defined it as the "additional information processing performed when multiple, connected actors pursue goals that a single actor pursuing the same goals would not perform." Malone and Crowston (1994) suggested that coordination is "the process of managing dependencies among activities."

We define intrinsic meta-modeling coordination as an iterative and collaborative process of adjusting individual sub-system's decisions and constraints in response to observable or measurable changes in shared state variables, in order to achieve more satisficing system of systems solutions.

In a system of systems with $i$ interdependent sub-systems, each sub-system is controlled by its own decision makers $\mathrm{DM}_{\mathrm{i}}$. Typically, $\mathrm{DM}_{\mathrm{i}}$ 's compute Pareto-optimal solutions for their local multi-objective problems without explicitly accounting for sub-system interdependencies in their decision-making processes, essentially acting as if each sub-system were completely independent of the other sub-systems. However, this disregard for the sub-system interdependencies leads to system of system solutions that might not be acceptable to all stakeholders over the entire planning horizon, as was illustrated through the surface transportation example in Chapter 1.

Since decisions made in interdependent sub-systems affect shared state variables, an uncoordinated decision making process could result in levels of shared state variables that are not acceptable to all involved parties over a longer planning horizon. Thus, ideally, stakeholders would like to have a process that enables them to assess the impact that decisions made in different sub-systems have on shared state variables, and to paraphrase Singh (1992), harmoniously adjust their local sub-system optimizations in order to achieve global system of systems goals. This can be accomplished through the use of intrinsic meta-modeling. Figure 12 illustrates a system of systems composed of $i$ sub-systems each of which is characterized by a local multi-objective problem. Although each sub-system is controlled by independent $\mathrm{DM}_{\mathrm{i}}$,'s, 
their coordination is imperative to achieve solutions that are sustainable and acceptable to all stakeholders in the long run.

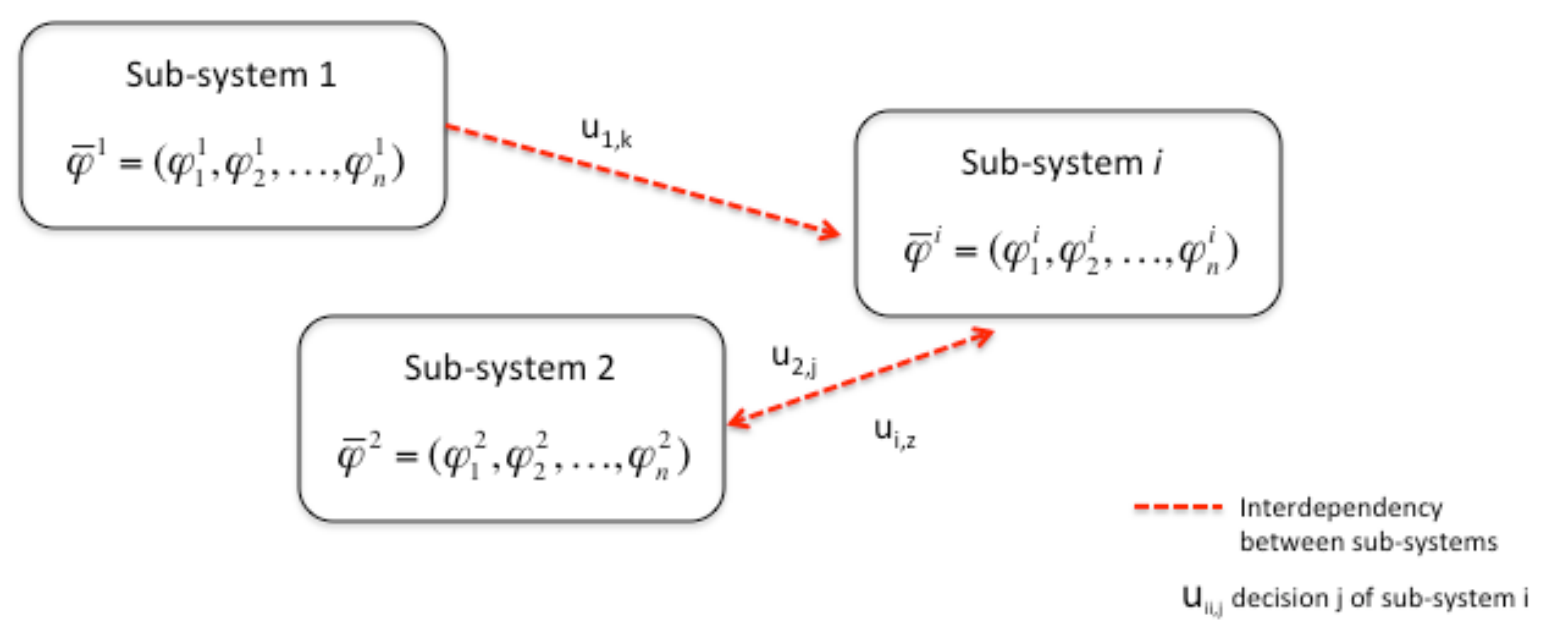

Figure 12: A system of systems in which each sub-system is characterized by its own multi-objective problem. Decisions made in sub-system 1 affect sub-system $i$, while decisions made in sub-systems $i$ and 2 affect each other.

With respect to Figure 12, $\bar{\varphi}^{i}$ represents a vector of objectives of sub-system $i$, where $n$ denotes its dimension. Eq. 4-4 suggests that $\bar{\varphi}^{i}$ is a function not only of the inputs and state variables of sub-system $i$, but is also a function of some subset of the circle of influence of subsystem 1 (circle of influence was defined in Chapter 1). Hence, $\bar{\varphi}^{i}$ is influenced by that subset of the decisions made by $\mathrm{DM}_{1}$ and inputs in sub-system $1,\left\{u^{1}(t)\right\}$, which affect state variables that are shared between sub-systems $i$ and 1. Based on Haimes' (2012) definition of system of systems, $\bar{s}^{i}(t) \cap\left\{s^{1}(t)\right\}=s s_{m}(t)$ (as shown in Eq. 4-4) (i.e., there exists at least one shared state variable $\mathrm{ss}_{\mathrm{m}}$ ), therefore local sub-system level optimizations are interdependent and must somehow be coordinated. 


$$
\begin{aligned}
\bar{\varphi}^{i} & =f\left(\bar{y}^{i}(t)\right) \\
& =f\left(\bar{u}^{i}(t), \bar{s}^{i}(t), \Phi_{1}\left(C_{I}^{1}(t)\right)\right) \\
& =f\left(\bar{u}^{i}(t), \bar{s}^{i}(t), \Phi_{1}\left(\left\{u^{1}(t)\right\},\left\{s^{1}(t)\right\}\right)\right) \\
& =f\left(\bar{u}^{i}(t), \bar{s}^{i}(t), s s_{m}(t)\right)
\end{aligned}
$$

Given the sub-system interdependencies, in order to achieve global system of systems solutions that are more acceptable to all stakeholders over the entire planning horizon we need a higher-level coordinator that can harmonize the sub-system specific knowledge and information and facilitate the process of collaborative coordination of sub-systems' decisions (see Figure 13). The intrinsic meta-model, introduced in Section 4.4, can serve as that higher-level coordinator. Based on its role as a higher-level coordinator we call the intrinsic meta-model, phantom metalevel decision maker $\left(\mathrm{DM}_{0}\right)$ (where phantom suggests that this $\mathrm{DM}_{0}$ does not exist in reality, but was artificially created to facilitate the coordination). The meta-model (i.e., $\mathrm{DM}_{0}$ ) represents the integrated (global) collection of knowledge about a system of systems from all modeling perspectives. In this construct, the role of the $\mathrm{DM}_{0}$ is to provide continuous feedback to $\mathrm{DM}_{\mathrm{i}}$ 's about the current range of values of the shared state variables at some discrete time $k(k \in t)$ (where the current range of values for shared state variables at time $k$ reflects the aggregate impact of sub-systems' decisions on shared states from $k-1$ to $k$ ), as well as about the sub-system specific ranges of acceptable values of the shared state variables at some time $k$ (see Figure 13). 


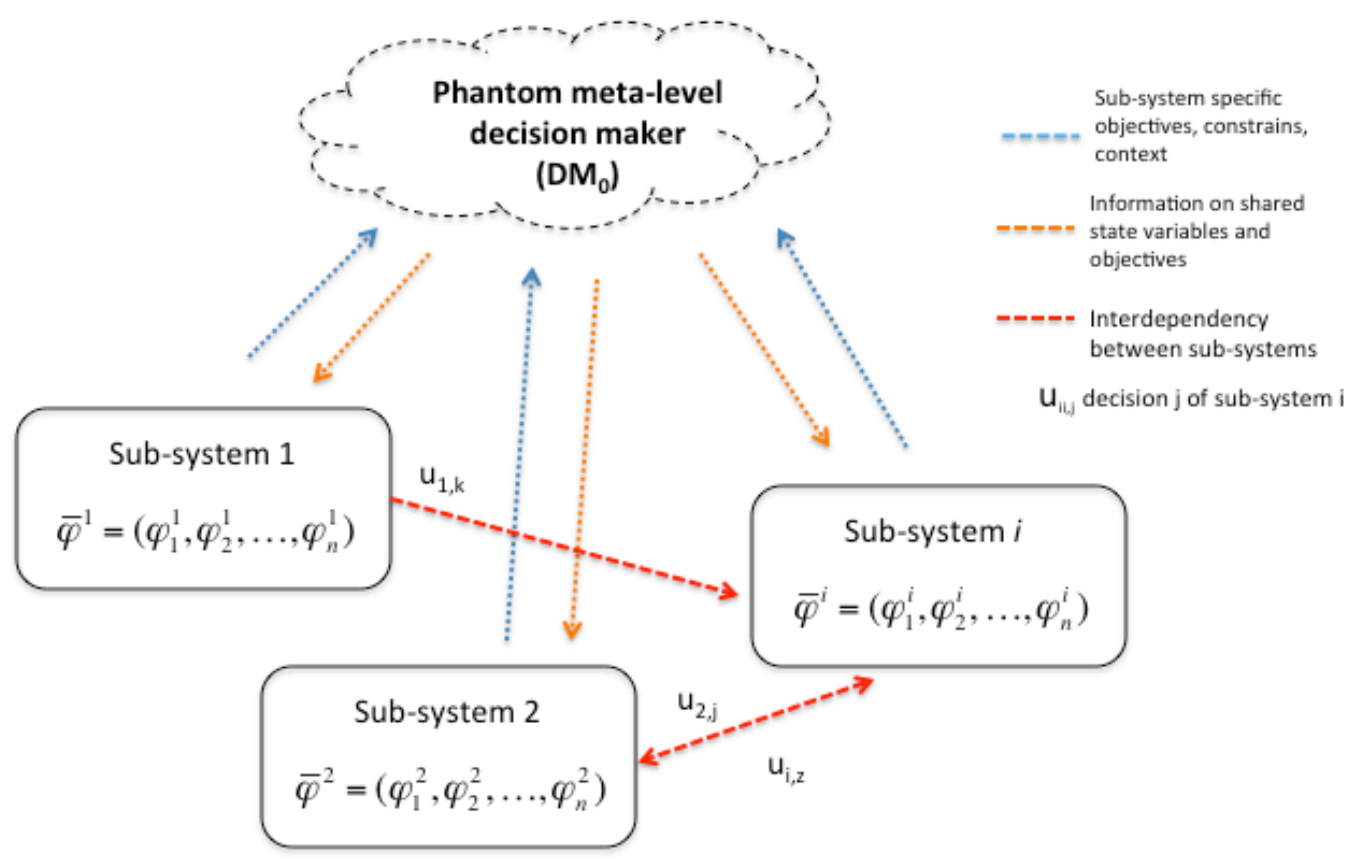

Figure 13: Meta-model $\left(\mathrm{DM}_{0}\right)$ facilitates the identification of shared states and objectives among the interdependent sub-systems, and sends that information to individual sub-systems.

For each shared state variable, there must exist some overlap between the sub-system specific ranges, and at the meta-level that overlap is defined as the globally acceptable range of values of shared state variables (discussed in more detail later in this section). Based on this knowledge, individual $\mathrm{DM}_{\mathrm{i}}$ 's develop sub-system specific coupling functions that link shared states to other non-shared essential variables of each sub-model (see Eq. 4-5 for a general expression, and see Chapter 5 for specific examples).

coupling function $=g_{i}\left(s s_{m}^{i}(t), \bar{s}^{i}(t), \bar{u}^{i}(t)\right)$

Because of the coupling functions linking shared state variables to other essential variables of each sub-model, $\mathrm{DM}_{\mathrm{i}}$ 's might be able to better identify those factors (i.e., decisions, constraints) that ought to be adjusted at the individual sub-system level, or through a collaborative effort, in order to reach a range of values of shared state variables that would be more acceptable to all $\mathrm{DM}_{\mathrm{i}}$ 's over the entire planning horizon (Figure 14). For a problem involving the maintenance of the bridge infrastructure, these changes to the sub-systems' decision spaces might include: (i) 
innovative funding mechanisms (e.g., private-public partnerships, tolls, usage taxes) that would require some or all infrastructure users to participate in infrastructure maintenance financing; (ii) incentives and/or commitment devices that would encourage regional industries to support employee telecommuting or to participate in maintenance funding by paying infrastructure usage fees for trucks that exceed a certain weight.

Based on the feedback received from the meta-model, at the level of each sub-system, $\mathrm{DM}_{\mathrm{i}}$ 's must re-evaluate their objective functions and seek satisficing solutions based on the suggested changes from the other sub-systems, such that $\bar{\varphi}^{i} \leq \bar{\varepsilon}^{i}$ where $\bar{\varepsilon}^{i}=\bar{y}^{i^{*}}-\bar{A}^{i}$ (the satisficing solution for objective $\varphi^{i}$ falls within an acceptable range $\varepsilon^{i}$ from a Pareto-optimal solution $y^{i *}$; $\varepsilon^{i}$ is calculated as the difference between the Pareto-optimal solution and some locally defined aspiration level $\mathrm{A}^{\mathrm{i}}$ ). We mentioned in Section 4.2 that satisficing refers to achieving a solution that is at least as good as some pre-defined aspiration level. Determining this aspiration level is not an easy endeavor, but sometimes it can be guided by externalities that are not under the influence of decision makers. For example, when determining the aspiration levels for the objectives in the social and economic sub-systems in Chapter 5, we recognize that these subsystems might have target values that are imposed externally - a certain number of noncommercial travelers must travel to work on a daily basis to maintain the operation of the regional commerce; a large competitive market might require a more reliable manufacturing supply delivery process than a smaller competitive market, hence this will determine the minimum required number of commercial vehicles that should safely cross the bridge at some period of time $\mathrm{k}$. 


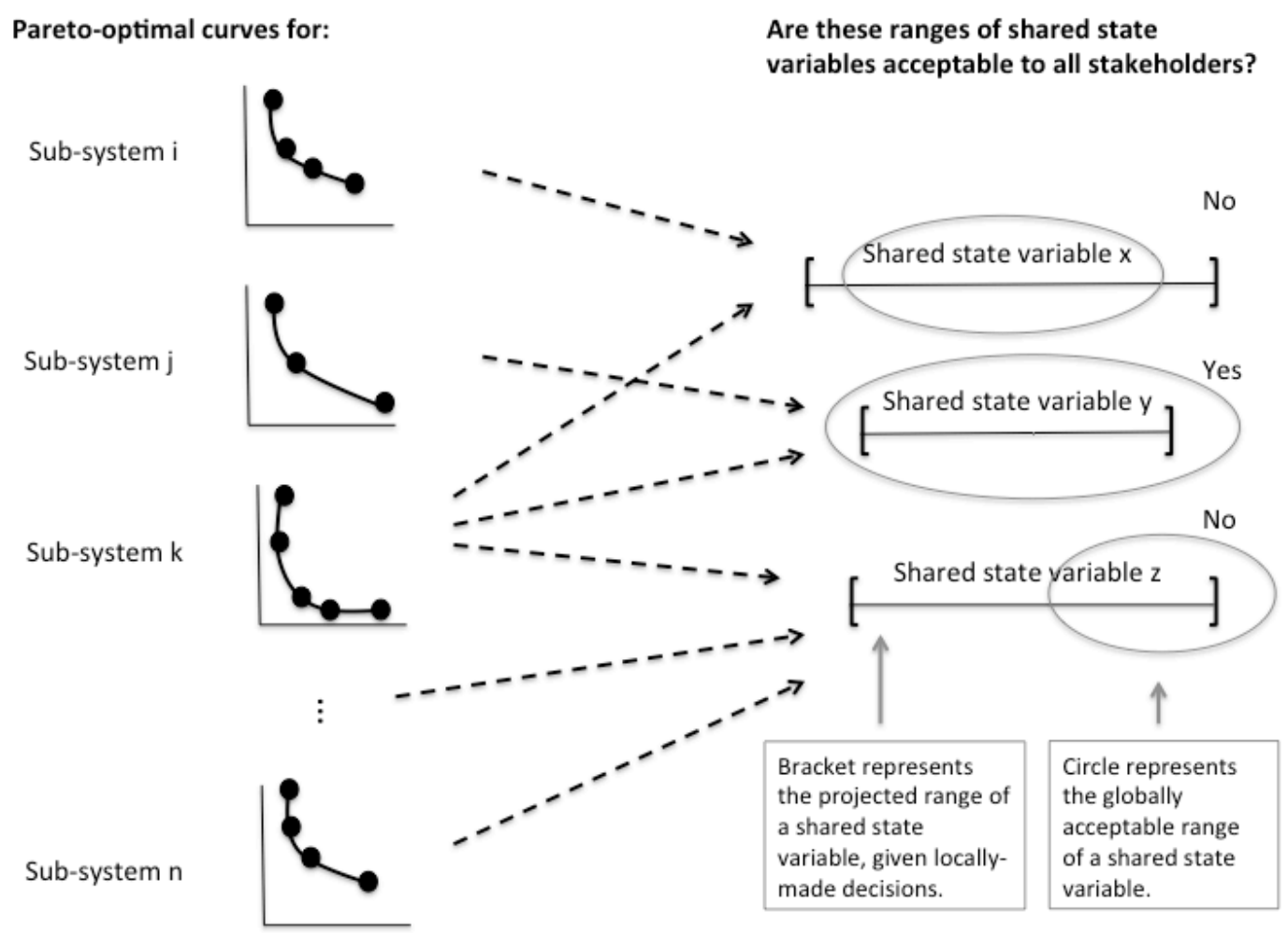

Figure 14: $\mathrm{DM}_{\mathrm{i}}$ 's can better understand the impact of their actions on the overall system of systems by assessing how their individual actions might impact the levels of shared state variables under some future emergent risk scenarios. If individual decisions made by different $D M_{i}$ 's result in values of shared state variables that are not acceptable to all $D M_{i}$ 's, then $D M_{i}$ 's can, with the help of the meta-model, identify some changes that could be made independently or collaboratively, and that would result in more acceptable values of shared state variables, while also enabling each $D_{M_{i}}$ to satisfice local objectives.

Based on the re-computed sub-system level satisficing results, new projected ranges of shared state variables must be re-evaluated. This process continues until a solution is found that satisfices all local sub-system objectives and results in levels of shared state variables that are acceptable to all $\mathrm{DM}_{\mathrm{i}}$ 's over the entire planning horizon. Given the evolving nature of the stakeholders' values, requirements and objectives, and the dynamic nature of the sub-systems, we recognize that long-term solutions, even through initially acceptable to all stakeholders, might become unacceptable to some or all stakeholders over time. For example, it has been documented (Haimes, 2009) that one might have a Pareto-optimal frontier with two objectives, but that with the addition of a third objective at some point in the future, some decisions on the original Pareto-optimal frontier may become inferior. Hence, the meta-modeling coordination 
process must be occasionally revisited and re-evaluated. In the end, a failure of sub-systems to agree on a globally-acceptable range of values of shared state variables could result in local subsystem difficulties that could, over time, propagate and become detrimental to the entire system of systems (for more information see Andrijcic et al. (2013)). Similar to the process of modeling the sub-systems, the process of identifying those changes that would allow all $\mathrm{DM}_{\mathrm{i}}$ 's to achieve local satisficing solutions while also achieving globally acceptable levels of shared state variables, is an iterative and learn-as-you-go process that requires continuous experimentation (as illustrated in Figure 15).

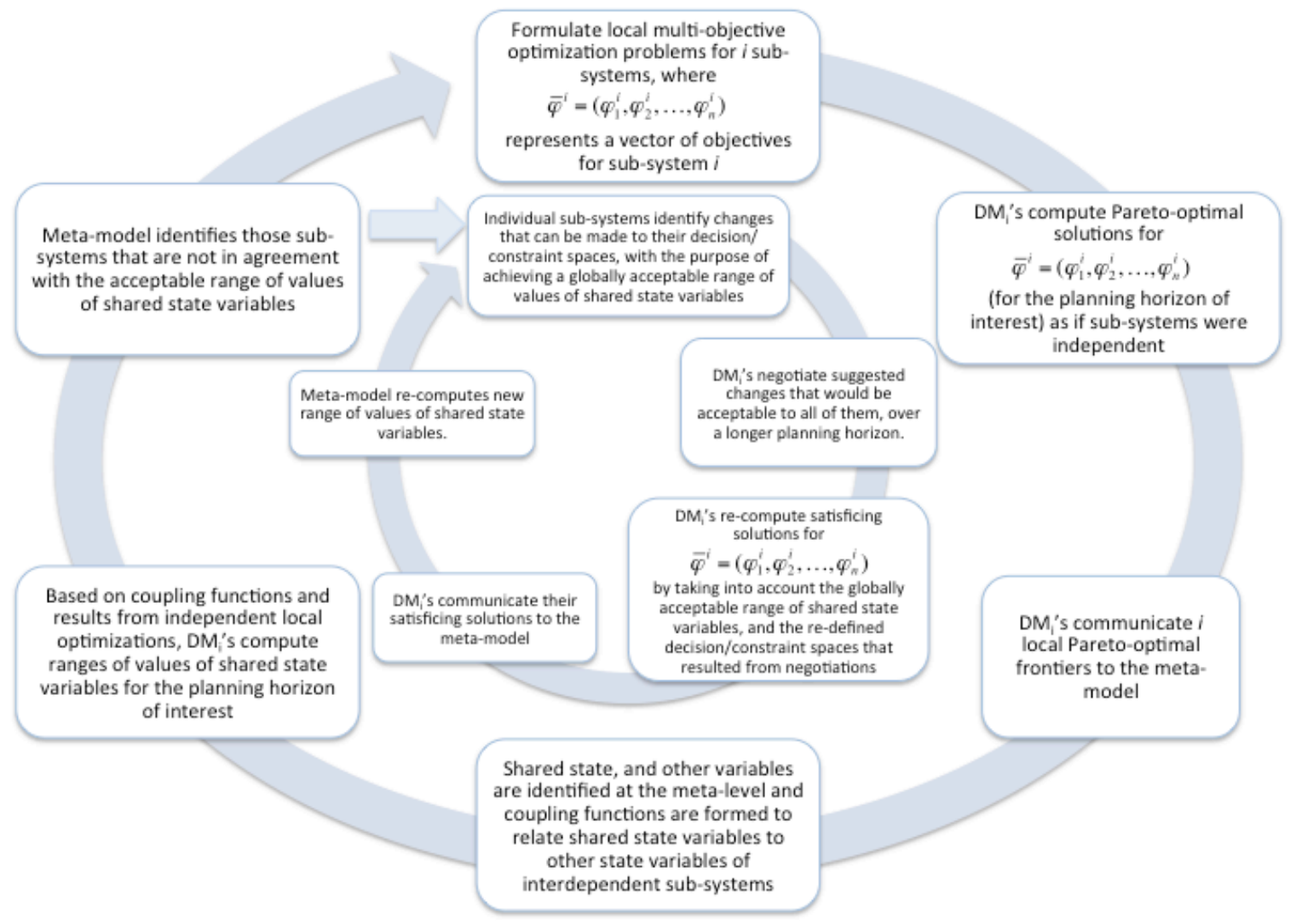

Figure 15: Iterative and collaborative meta-modeling coordination process

A specific example of the coordination process is given in Chapter 5. For now we introduce a generalized example. Assume we are dealing with a system of systems that we are modeling from two modeling perspectives, and we represent it through two sub-models. For simplicity, we 
further assume that the system under consideration falls into the category of linear time-invariant systems that we evaluate at discrete time intervals $k$, that the noise parameters $\left(\omega_{j}^{i}(k), v^{i}(k)\right)$ are negligible, and that the two sub-models representing this system are described by Eqs. (4-6) and (4-7). In Eqs. 4-6 and 4-7, and in Figure 16, $s_{j}^{i}$ represents a state variable $j$ belonging to subsystem $i ; u_{j}^{i}$ represents the input/decision $j$ of sub-system $i ; \omega_{j}^{i}$ and $v_{j}^{i}$ represent white noise introduced into the sub-system $i ; y^{i}$ represents the output of sub-system $i$.

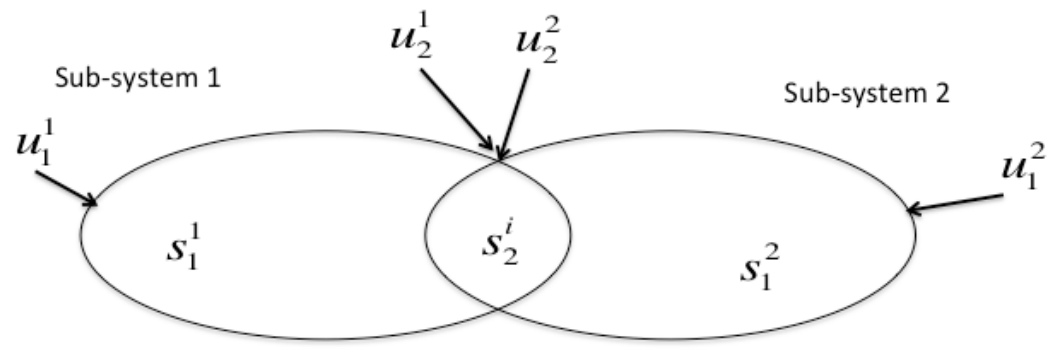

Figure 16: Two sub-systems sharing a single state variable

We assume that there is a single shared state variable connecting these two sub-models, namely state variable $s_{2}^{i}$ (see Eq. 4-8). We furthermore assume that there exists a single objective in each sub-system, as expressed in Eqs. 4-6 and 4-7, and we note that both objectives are functions of the shared state variable. We also notice that the shared state variable is computed differently in each sub-system (based on the local context and information). For this system of systems to be operational over the entire planning horizon, Eq. 4-2 must hold. In order for Eq. 42 to hold, relevant stakeholders and decision makers must iteratively reassess $u_{2}^{i}$ for $i=1,2$ until they reach a level of shared state variable that is acceptable to both sets of stakeholders over the entire planning horizon, while satisficing their local objectives (see Eqs. 4-9 to 4-12). This iterative process is summarized in Figure 17. 


\section{Sub-system 1:}

$$
\begin{aligned}
& \left\{\begin{array}{l}
s_{1}^{1}(k+1)=a_{1}^{1} s_{1}^{1}(k)+b_{1}^{1} u_{1}^{1}(k)+\omega_{1}^{1}(k) \\
s_{2}^{1}(k+1)=a_{2}^{1} s_{2}^{1}(k)+b_{2}^{1} u_{2}^{1}(k)+\omega_{2}^{1}(k) \\
y^{1}(k)=c_{1}^{1} s_{1}^{1}(k)+c_{2}^{1} s_{2}^{1}(k)+v^{1}(k)
\end{array}\right. \\
& \varphi^{1}=\min y^{1} \\
& \quad=\min f_{1}\left(u_{1}^{1}(k), u_{2}^{1}(k) s_{1}^{1}(k), s s_{2}(k)\right)
\end{aligned}
$$

\section{Sub-system 2:}

$$
\begin{aligned}
& \left\{\begin{array}{l}
s_{1}^{2}(k+1)=a_{1}^{2} s_{1}^{2}(k)+b_{1}^{2} u_{1}^{2}(k)+\omega_{1}^{2}(k) \\
s_{2}^{2}(k+1)=a_{2}^{2} s_{2}^{2}(k)+b_{2}^{2} u_{2}^{2}(k)+\omega_{2}^{2}(k) \\
y^{2}(k)=c_{1}^{2} s_{1}^{2}(k)+c_{2}^{2} s_{2}^{2}(k)+v^{2}(k)
\end{array}\right. \\
& \varphi^{2}=\min y^{2} \\
& \quad=\min f_{2}\left(u_{1}^{2}(k), u_{2}^{2}(k) s_{1}^{2}(k), s s_{2}(k)\right)
\end{aligned}
$$

\section{Shared state variable:}

$$
\begin{aligned}
s s_{2}(k) & =\bigcup\left(s_{2}^{1}(k), s_{2}^{2}(k)\right) \\
& =g\left(s_{2}^{1}(k), u_{2}^{1}(k), s_{2}^{2}(k), u_{2}^{2}(k)\right)
\end{aligned}
$$

s.t.

$u_{2}^{1}(k) \neq u_{2}^{2}(k)$ 
At the meta-level we determine the globally acceptable range of shared state variables, based on collective knowledge from all sub-systems. This globally acceptable range is the intersection of the locally acceptable ranges of values of the shared state variable (see Eqs. 4-9 and 4-10), where $a_{l}^{i}$ represents the lower bound of the locally acceptable range, and $a_{h}^{i}$ represents the upper bound.

$s s_{2}(k)=\bigcup\left(s_{2}^{1}(k), s_{2}^{2}(k)\right)=g\left(s_{2}^{1}(k), u_{2}^{1}(k), s_{2}^{2}(k), u_{2}^{2}(k)\right)$

$a_{l} \leq s s_{2}(k) \leq a_{h}$

s.t.

$\left[a_{l}, a_{h}\right]=\left[a_{l}^{1}, a_{h}^{1}\right] \bigcap\left[a_{l}^{2}, a_{h}^{2}\right]$

Based on the feedback from the meta-model about the globally acceptable ranges of shared state variables, $\mathrm{DM}_{\mathrm{i}}$ 's reassess their local objectives and satisfice based on locally determined aspiration levels $\mathrm{A}^{\mathrm{i}}$ (see Eqs. 4-11 and 4-12).

$$
\min _{U^{1}}\left|s s_{2}(k)-s_{2}^{1}(k)\right|
$$

s.t.

$\bar{\varphi}^{1} \leq \bar{\varepsilon}^{1}$

where

$\bar{\varepsilon}^{1}=\bar{y}^{1^{*}}-\bar{A}^{1}$

$\min _{U^{2}}\left|s s_{2}(k)-s_{2}^{2}(k)\right|$

s.t.

$\bar{\varphi}^{2} \leq \bar{\varepsilon}^{2}$

where

$\bar{\varepsilon}^{2}=\bar{y}^{2^{*}}-\bar{A}^{2}$ 


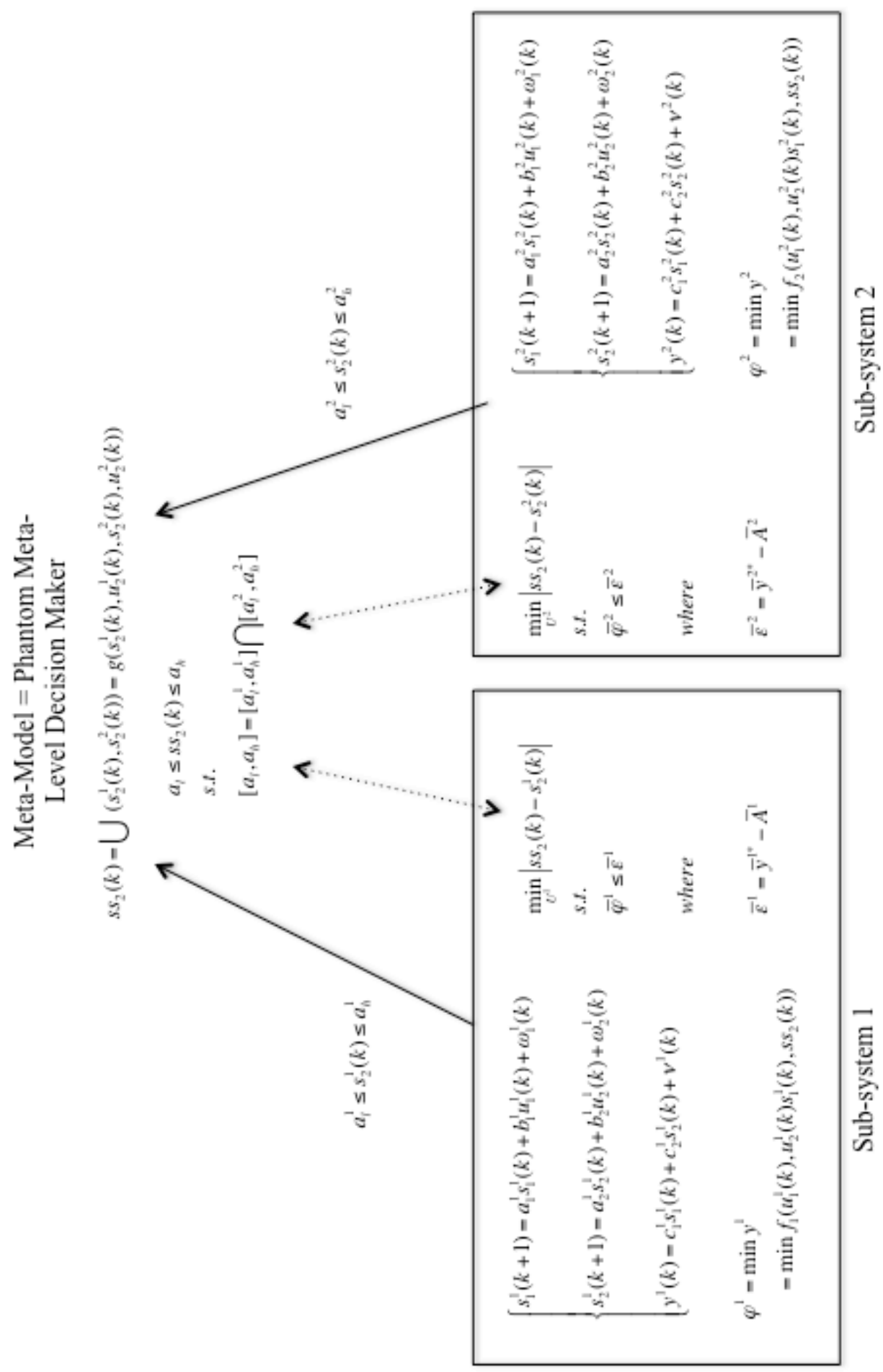

Figure 17: Summary of the meta-modeling coordination for a system of systems composed of two linear time invariant sub-systems 


\subsection{Brief summary}

This Chapter presented extensions of Haimes' Phantom System Models intrinsic meta-modeling theory (Haimes, 2012), by providing operational guidelines for the construction of sub-models and the meta-model, and by introducing a formal meta-modeling coordination process by which, through the use of coupling functions, meta-model and satisficing conditions, we can handle distributed optimization problems. In particular, this dissertation presented: (i) an iterative and learn-as-you go methodological process for modeling systems of systems which takes advantage of shared state variables, and (ii) a process for a collaborative coordination of decentralized subsystems' decisions through the meta-model. The phases of the methodological process for modeling systems of systems are deliberately generic, so as to provide some general guidelines and insights for a wide variety of systems of systems, without trivializing the complexity of the modeling process. In the proposed process special emphasis is placed on utilizing shared state variables, which in essence are phantom variables in that they are determined from a set of constrained minimization conditions over a common interval. While any variables that might be shared among sub-systems are important to consider in the modeling process, state variables are directly impacted by decision variables, thus providing stakeholders and decision makers an opportunity to explore the impacts of decentralized yet interdependent decisions that are made independently in different sub-systems. In the following chapter, this methodological process for modeling and coordination is applied to a bridge infrastructure maintenance example. 


\section{Application of Intrinsic Meta-Modeling to a Bridge Infrastructure System of Systems}

Chapter 4 introduced the methodological roadmap for creating the sub-models and the metamodel for a system of systems and it introduced the idea that intrinsic meta-modeling can be used as an instrument for coordination of decisions made independently in decentralized subsystems. In this Chapter we will apply the developed theory and methodology to a specific system of systems problem. In particular, we will model a strategically important bridge of interest as a system of systems, composed of natural, physical and engineered elements; commercial and non-commercial bridge users; and local-, state-, and federal-level decision makers, operating at the intersection of the social, economic, organizational, environmental and technological spheres of influence.

\subsection{The problem}

Insufficient and untimely bridge maintenance practices, coupled with rising travel demands and freight loads, increase the rate of bridge deterioration over time, and result in a reduction of the bridge load carrying capacity (live load that a structure can safely carry). This, in turn, increases travel time and costs for commercial and non-commercial bridge users, and can cause significant socio-economic ripple effects in the region of concern (see Figure 18).

Presently, for maintenance purposes, bridges are primarily modeled and managed as solely physical systems. Bridge maintenance decisions are often not made until significant structural failures occur, and fixes are often short-term, excluding a serious consideration of the impact of current decisions on: (i) future maintenance options, and (ii) other stakeholders (e.g., commercial and non-commercial infrastructure users). The general disregard for the potential impacts of current decisions on future maintenance options, and on other stakeholders, often reduces the set of available future maintenance alternatives because the structural condition worsens to the point that only more aggressive maintenance efforts are feasible. We suggest that a more sustainable 
management effort should recognize: (i) that bridge infrastructure systems are socio-technical systems of systems, in which decisions made in one sub-system affect the states of interdependent sub-systems; (ii) that this system of systems is characterized by a diverse set of stakeholders whose changing needs and constraints evolve over different time periods. To support the development and implementation of such a management approach, we need a process through which we can assess these evolving and often conflicting concerns in a comprehensive and integrative way, over the entire planning horizon.

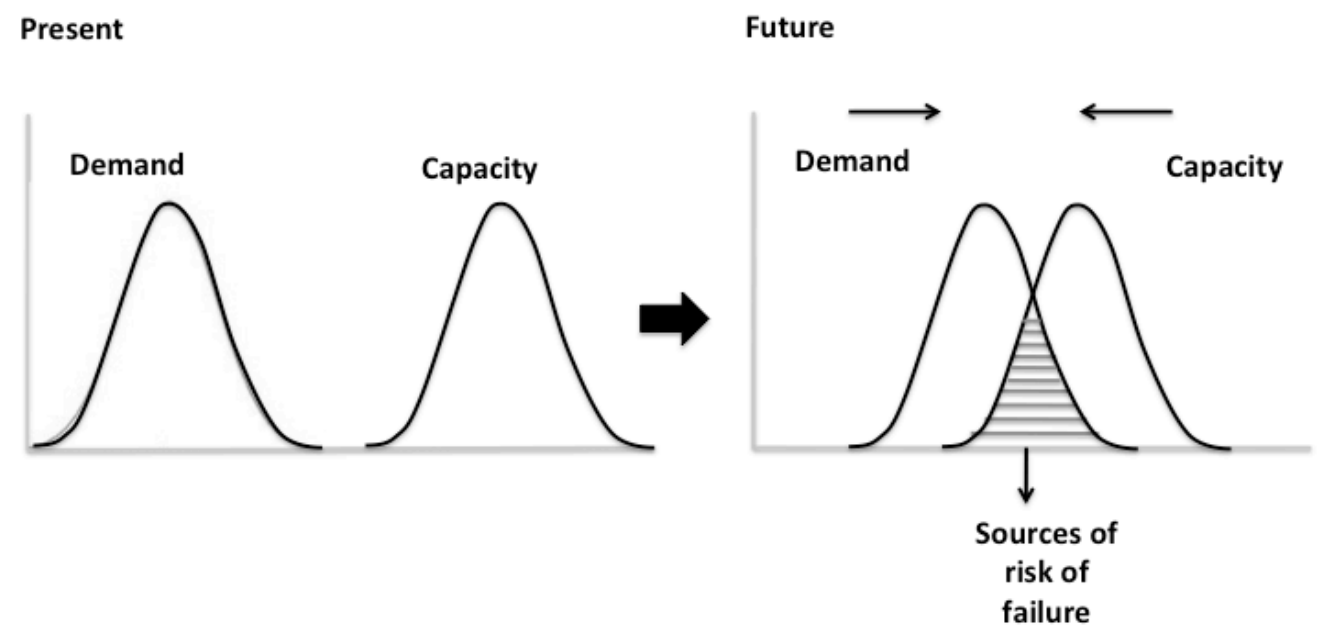

Figure 18: Over time, with untimely or insufficient bridge maintenance, bridge traffic capacity decreases while traffic demand on a bridge increases. This creates sources of social and economic risks.

Therefore, the goal of this case study is to demonstrate the value of intrinsic meta-modeling in creating a management environment in which relevant stakeholders can assess the impacts of maintenance options over time, from multiple perspectives, with the goal of creating more sustainable, collaborative, and forward-looking practices that can better anticipate, and be more resilient to, future emergent changes. To avoid a purely theoretical discussion, we focus our case study on the Hampton Roads Bridge Tunnel (HRBT) located in the Hampton Roads Area of Southeastern Virginia.

It is important to note that the purpose of this Chapter is not to provide concrete maintenance solutions for the studied bridge, nor to provide detailed mathematical models representing 
different sub-systems of a bridge infrastructure, but, instead, to illustrate the potential value of modeling the bridge infrastructure via the intrinsic meta-model, by coordinating multiple systemand stakeholder-level perspectives. Details about the theory and methodology applied in this Chapter are discussed in Chapter 4, and the broader implications of adopting such an approach are discussed in Chapter 6. Although the focus of this dissertation is on the U.S. bridge infrastructure, the discussed theory and methodology could be applied to other civil infrastructure systems of systems, thus addressing the need for approaches that are generic and transferable between different infrastructures (Jamshidi, 2009).

\subsection{The strategic importance of the Hampton Roads Bridge Tunnel (HRBT)}

The Hampton Roads Bridge Tunnel (HRBT) is a 3.5-mile long structure that carries Interstate 64 and connects the City of Norfolk with the City of Hampton in Southeastern Virginia. As its name suggests, it is composed of bridge trestles, manmade islands, and tunnels that are located under important shipping channels in the Hampton Roads Harbor (Roads to the Future, 2007). HRBT is composed of two 12-foot wide lanes in each direction - these are carried by different structures. The original structure (westbound) was put into operation in November of 1957, while the eastbound structure was constructed later and put into operation in June of 1976 (Roads to the Future, 2007).

The westbound and eastbound tunnels are each 7,479 feet long, and at the deepest point they are 108 feet below sea level. The roadways carried by the bridge structure are at an elevation of 17.5 feet above the average high tide sea level. The structure rests on two manmade islands that transition the roadway between bridge to tunnel, and these islands were built at 11 feet above the average high tide sea level (Roads to the Future, 2007). The tunnel structures were built by the "immersed sunken tube method, comprised of shipyard-built prefabricated tunnel elements each about 300 feet long, placed by lay-barges and joined together in a trench dredged in the bottom of the harbor, and backfilled over with earth" (Roads to the Future, 2007). The HRBT is a tollfree facility operated by the Virginia Department of Transportation (VDOT). The facility is operated through a state-of-the-art computer traffic control system and a video monitoring 
system. Major renovation was conducted in 1998 through 1999 to provide major rehabilitation to the substructure, widen the shoulders, and build a new bridge deck (Roads to the Future, 2007).

In addition to its complex structure, the HRBT is of interest in this case study primarily because of its strategic location. It is located in a multimodal area (with a population exceeding 1.6 million) that is adjacent to the Atlantic Ocean and Chesapeake Bay and that serves large consumer markets all along the East Coast of the U.S (Hampton Roads Planning District Commission, 2007). In addition to boasting one of the largest and most successful international seaports on the East Coast (it ranks as the $3^{\text {rd }}$ largest U.S. East Coast Port in terms of general cargo), the Hampton Roads Area is also a center of military and naval installations, thus it is an important strategic location:

Hampton Roads is not only one of the largest commercial freight hubs along the U.S. East Coast, but it is also home to many military facilities. This region contains approximately 110,000 active duty military, of which more than 80,000 are U.S. Navy. The total Department of Defense (DOD) population in Hampton Roads, including active duty, reserve component, retirees and family members totals approximately 300,000 in an area with a total population of 1.6 million. For this reason, it is imperative to maintain a safe, secure, and efficient transportation system in Hampton Roads should an unexpected event occur that would require a rapid deployment of military cargo and personnel via air, land, or sea. (Hampton Roads Planning District Commission, 2007)

According to a Hampton Roads Planning District Commission report (2007), in 2004, 48\% of all domestic freight entering Hampton Roads, and 73\% of freight leaving Hampton Roads was transported by trucks, resulting in approximately 20,000 trucks entering and leaving the area each weekday. Of these, approximately $46 \%$ or 4,800 trucks crossed over the HRBT each weekday. In total, over 95,000 vehicles crossed the HRBT each weekday in 2005, as compared to 56,000 vehicles that crossed the Monitor Merrimack Memorial Bridge Tunnel and 30,000 vehicles that crossed the James River Bridge. Due to its strategic importance for the transportation of commercial and non-commercial goods and people, the Hampton Roads Planning District Commission suggests, "In order for Hampton Roads to remain competitive in 
attracting new business interests and continue to grow economically, its transportation network must facilitate the rapid and efficient movement of raw materials and finished products using trucks, trains, ships, and planes” (Hampton Roads Planning District Commission, 2007).

However, the Hampton Roads Area is beginning to lag in this goal, as in 2005 approximately 20,000 overweight trucks were turned around at regional tunnels. The westbound HBRT had the most overweight vehicles, with $69 \%$ of all overweight vehicles being turned around at that facility (Hampton Roads Planning District Commission, 2007). The Hampton Roads Transportation Report Card issued by the Norfolk Branch of the American Society for Civil Engineers (ASCE) suggests that the overall state of the transportation infrastructure in the Hampton Roads leaves much to be desired. The report gives roads in the region a grade of Dand bridges a grade of $\mathrm{C}+($ Norfolk ASCE, 2005). The report suggests, "73 bridges in Hampton Roads are load posted, meaning that less than the normal loads are allowed to cross." Furthermore, the same report suggests the following:

- $27 \%$ of all bridges in Hampton Roads are structurally deficient or functionally obsolete;

- 432 bridges in Hampton Roads have a major component with a condition rating of 5 or less, indicating some rehabilitation is needed;

- Repairing or replacing the structurally deficient or functionally obsolete Hampton Roads bridges, to bring them to acceptable level, would cost approximately $\$ 500$ million;

- Poor transportation conditions make Hampton Roads the nation's $2^{\text {nd }}$ worst tourist destination for summer traffic delays;

- HRBT is considered severely congested with no funded improvements.

As the condition of the transportation infrastructure, especially bridges, in the Hampton Roads Area deteriorates further, is it likely that the number of returned overweight trucks and the general congestion levels will be even greater. Thus, any bridge maintenance in the Hampton Roads Area should be influenced by the potential social and economic costs caused by insufficient or untimely maintenance. The Norfolk branch of ASCE suggests, "Investment is critical in solving our transportation crisis, but only part of a resourceful, complete strategy that incorporates demand management, new technologies, responsible community growth and real commitment from policy leaders and the public" (Norfolk ASCE, 2005). 


\subsection{Modeling the bridge infrastructure as a system of systems}

We define a system of systems as a collection of interdependent sub-systems, each of which is connected to at least one other sub-system through one or more shared state variables (Haimes, 2012). With that in mind, we describe the HRBT as a system of systems, composed not only of physical sub-systems (i.e., the engineered components), but also of sub-systems that reflect the socio-economic environment within which the infrastructure operates (i.e., federal-, state-, and local- level policymakers and decision makers, regional industries, non-commercial infrastructure users). This representation is appropriate given that these stakeholders frame the extent of possible solutions in bridge infrastructure maintenance and management.

The stakeholders under consideration are naturally concerned with different aspects of the bridge infrastructure, and they make decisions in a decentralized and distributed manner, often not realizing the impact that their own decisions have on the interdependent sub-systems. Decision makers from Departments of Transportation (DOTs) and Metropolitan Planning Organizations (MPOs) are primarily concerned with the dynamic changes to the bridge condition and load ratings, the cost of maintenance to address deterioration issues, as well as the political environment and influences. They would like to maximize bridge condition and load ratings, while minimizing the net present value (NPV) of their maintenance investments. Noncommercial bridge users are generally concerned about maximizing their accessibility to places of interest and minimizing their cost of travel. Similarly, commercial users rely on the bridge infrastructure for efficient and affordable supply chain management, hence they are concerned about maximizing their accessibility to places of interest, minimizing their cost of travel, and maximizing the loads they are allowed to carry.

In the presence of this decentralized multi-objective problem, we would like to understand how these different stakeholders could work together to accomplish their own objectives while being explicitly cognizant and responsive to the needs and objectives of the other stakeholders, and the overall system of systems. 


\subsection{Formulation of the problem}

We assume that major maintenance decisions for the HRBT are made every two years, following a bridge inspection, and we would like to understand which of the available long-term maintenance policies (shown in Table 3) are most acceptable to all stakeholders over a 24-year horizon (starting in 2011, ending in 2035). The stakeholders under consideration include decision makers from Virginia DOT (VDOT) and Hampton Roads MPO, as well as non-commercial and commercial bridge users. For illustrative purposes we have created five alternative maintenance policies shown in Table 3. These policies are not based on actual maintenance plans for the HRBT, but are but are merely illustrative examples, and they range from an end-loaded policy in which maintenance is performed only at the end of the planning horizon when the condition of the bridge has deteriorated significantly, to a front-loaded policy in which preventative measurements are taken immediately and no follow-up maintenance is performed, to several types of distributed policies in which investments are made at different points in time during the planning horizon.

\begin{tabular}{|c|c|c|c|c|c|c|c|c|c|c|c|c|}
\hline & \multicolumn{7}{|c|}{ Maintenance Investment (in Smillion) } & & \\
\hline Policy & $\mathbf{u ( 1 )}$ & $\mathbf{u}(\mathbf{2})$ & $\mathbf{u}(\mathbf{3})$ & $\mathbf{u}(\mathbf{4})$ & $\mathbf{u}(\mathbf{5})$ & $\mathbf{u}(\mathbf{6})$ & $\mathbf{u}(\mathbf{7})$ & $\mathbf{u}(\mathbf{8})$ & $\mathbf{u}(\mathbf{9})$ & $\mathbf{u}(\mathbf{1 0})$ & $\mathbf{u}(\mathbf{1 1})$ & $\mathbf{u}(\mathbf{1 2})$ \\
\hline $\begin{array}{c}\text { 1: End } \\
\text { Loaded }\end{array}$ & 0.00 & 0.00 & 0.00 & 0.00 & 0.00 & 0.00 & 0.00 & 0.00 & 0.00 & 0.00 & 0.00 & 10.00 \\
\hline $\begin{array}{c}\text { 2: Equal } \\
\text { Annual } \\
\text { Distribution }\end{array}$ & 0.83 & 0.83 & 0.83 & 0.83 & 0.83 & 0.83 & 0.83 & 0.83 & 0.83 & 0.83 & 0.83 & 0.83 \\
\hline $\begin{array}{c}\text { 3: Front } \\
\text { Loaded }\end{array}$ & 10.00 & 0.00 & 0.00 & 0.00 & 0.00 & 0.00 & 0.00 & 0.00 & 0.00 & 0.00 & 0.00 & 0.00 \\
\hline $\begin{array}{c}4: \\
\text { Distributed } \\
\text { Front } \\
\text { Loaded }\end{array}$ & 5.00 & 0.00 & 5.00 & 0.00 & 0.00 & 0.00 & 0.00 & 0.00 & 0.00 & 0.00 & 0.00 & 0.00 \\
\hline $\begin{array}{c}5: \\
\text { Distributed }\end{array}$ & 2.50 & 0.00 & 0.00 & 2.50 & 0.00 & 0.00 & 2.50 & 0.00 & 0.00 & 2.50 & 0.00 & 0.00 \\
\hline
\end{tabular}

Table 3: Maintenance decisions are made every 2 years, following a bridge inspection. This table indicates investment amounts (in \$million) at each discrete decision point, $u(1)=2011, u(12)=2033$. The total amount of investment over the planning horizon is the same for all alternatives (\$10 million). 


\subsection{The intrinsic meta-modeling process}

In Chapter 4 we described the intrinsic meta-modeling process, which enables us to collaboratively analyze a system of systems from multiple modeling perspectives. Generally this process would be implemented through the efforts of a cross-disciplinary group of relevant stakeholders and experts. In the absence of such a cross-disciplinary group, in this case study we use an arbitrary, yet convenient decomposition to represent the HBRT system of systems. Namely, we focus on the engineering, social, and economic modeling perspectives. We recognize that there are other valuable modeling perspectives, but argue that our choice is adequate to demonstrate the validity of the intrinsic meta-modeling theory and the application of the methodological steps. We recognize that each of the three modeling perspectives represents a fraction of the entire system of systems, and argue that the harmonization of the three modeling perspectives through shared state variables at the meta-level results in a better understanding of the system of systems to ultimately formulate public policy that is grounded on science, technology and socio-economic concerns. Each of the three modeling perspectives represents a collection of supporting sub-models, which are mathematical representations of the sub-systems under study. In the subsequent paragraphs, we briefly describe the process of constructing the individual sub-models and of identifying essential shared state variables. This is followed by a discussion on the harmonization of the three sub-models into a single meta-model via shared state variables. We conclude by discussing how the meta-model could be used to coordinate decisions made in interdependent sub-systems.

It is important to note that in the process of identifying the most critical system and stakeholder perspectives to be modeled we must first have an understanding of what questions we are trying to answer. For this case study we are more concerned with the long-term strategic view that considers how, in the long run, technical maintenance decisions made today affect the commercial and non-commercial users of the bridge in the future. In the absence of an interdisciplinary group of experts, we rely on existing literature to identify the set of representative engineering, social and economic sub-models that would answer the modeling questions of concern, and for each of the models identified in the literature we identify a set of relevant state, input, output, and decision variables. We then rely on existing literature to identify 
a set of representative databases (see Appendix III) that can be used to populate the relevant parameters.

\subsubsection{Constructing the engineering sub-model}

From a traditional engineering perspective, decision makers from DOTs and MPOs would like to spend the least amount of money possible to ensure that a bridge is structurally sound. The determination of structural health is not an easy one, as there are numerous physical components of a bridge (e.g., elements of substructure, superstructure, deck) that are affected by different deterioration processes. Thousands of civil engineering models have been developed to study those processes in detail, and the goal of this case study is not to try to replicate those. Instead, from an engineering perspective, we focus on the process of deterioration as it ultimately relates to changes in bridge load rating, as this will, in the end, greatly determine the ability of vehicles to travel over the bridge.

In the process of constructing the engineering sub-model, we identify a couple of models in the literature that model the macro-dynamic changes in the condition of the bridge over the planning horizon of interest, and from those models we identify major factors (variables) that would enable DOT and MPO decision makers to track these macro-dynamic changes over the time period of interest. Of the different types of bridge engineering models identified in literature (Andrijcic et al., 2012), of particular interest in this case study are statistical deterioration models, which provide the ability to forecast the effects of maintenance actions on the future condition of the bridge, thus they are appropriate for answering long-term and higher level questions about the general impacts of bridge maintenance decisions. Our selection of the representative statistical deterioration models is guided by our high-level modeling question: what are the broader social and economic implications of untimely or inadequate bridge maintenance? Hence, from the engineering perspective we are primarily concerned with the changes over time in the bridge condition rating and the bridge load rating, because these will determine the maximum possible utilization of the bridge over time. While changes in condition rating can be described in more detail by specifically addressing particular instances and causes of spalling, scour, cracking, etc., in this dissertation we are more interested in a higher level picture which enables us to relate how deterioration of the bridge affects the ability of people to travel over the bridge. Hence, we 
focus on models that ignore the detailed assessment of deterioration causes, and we focus instead on models that estimate changes in condition and load rating on a macro-scale. Condition ratings indicate the level of physical deterioration (e.g., cracking, scour, spalling, section loss, or other conditions) caused by environmental effects and traffic wear on the superstructure, substructure, and deck of a bridge (Dunker and Rabbat, 1995). Load rating indicates the live load capacity that a bridge can safely carry.

One of the engineering models of interest is a generalized linear regression model introduced by Chase et al. (1999), which estimates the changes to bridge condition rating of the superstructure, substructure and deck based on local climatic conditions, bridge age and average daily traffic. It utilizes data from the National Bridge Inventory (NBI) and the GIS database, introduced in Appendix III. Chase et al. (1999) provide regression equations for superstructure, substructure and deck deterioration, however, since in the long run, we wish to relate bridge condition rating to the bridge load rating, we only focus on the model formulation for bridge superstructure condition rating, represented by Eq. 5-1, where $\mathrm{C}=2.13$, and $\beta_{i} V_{i}$ are summarized in Table 4 (obtained from Chase et al. (1999)).

$$
S U P=E X P\left(C+\sum_{i} \beta_{i} V_{i}\right)
$$

This model assumes that (i) input variables represent most of the major factors contributing to bridge deterioration; (ii) the relation between bridge condition state and the input environmental factors can be modeled as a generalized linear function (i.e., nonlinearity doesn't cause significant prediction errors); (iii) there are only regular maintenance activities performed on the bridge; (iv) reconstructed bridges and the bridges with deterioration values below 3 are eliminated from the analysis (other outliers are removed as well). The essential variables associated with this model are given in Table 4. 


\begin{tabular}{|c|c|c|c|c|}
\hline I & Variables & Description & Coefficient & Value \\
\hline 1 & $\mathrm{~V}_{1}$ & Age [years] & $\beta_{1}$ & $-5.13 \times 10^{-3}$ \\
\hline 2 & $\mathrm{~V}_{2}$ & $\begin{array}{c}\text { Precipitation }\left[100^{\text {th }} \text { of }\right. \\
\text { inches }]\end{array}$ & $\beta_{2}$ & $-7.09 \times 10^{-4}$ \\
\hline 3 & $\mathrm{~V}_{3}$ & $\begin{array}{c}\text { Freeze-Thaw Cycle [\# } \\
\text { times per year] }\end{array}$ & $\beta_{3}$ & $-2.3 \times 10^{-3}$ \\
\hline 4 & $\mathrm{~V}_{4}$ & $\begin{array}{l}\text { Frequency of Salting }[\# \\
\text { times per year] }\end{array}$ & $\beta_{4}$ & $-7.44 \times 10^{-4}$ \\
\hline 5 & $\mathrm{~V}_{5}$ & Steel structure & $\beta_{5}$ & $-3.49 \times 10^{-2}$ \\
\hline 6 & $\mathrm{~V}_{6}$ & Timber structure & $\beta_{6}$ & $-2.35 \times 10^{-1}$ \\
\hline 7 & $\mathrm{~V}_{7}$ & Steel continuous structure & $\beta_{7}$ & $-3.87 \times 10^{-2}$ \\
\hline 8 & $\mathrm{~V}_{8}$ & $\begin{array}{c}\text { Concrete continuous } \\
\text { structure }\end{array}$ & $\beta_{8}$ & $-2.51 \times 10^{-2}$ \\
\hline
\end{tabular}

Table 4: Essential variables associated with the Chase et al. (1999) model

The second engineering model of interest is a linear regression model proposed by Chase and Gáspár (2000), which relates the superstructure condition rating to the bridge load rating (see Eq. $5-2$ ). The correlation coefficient of this model is 0.98 . The model assumes that: (i) only bridges where the load rating was calculated with HS loading were included; (ii) bridges identified as having undergone significant rehabilitation at some point in time were excluded; (iii) bridges with superstructure rating 0 were excluded; and (iv) the relationship between superstructure condition rating and average load rating is linear with almost constant error term variance, and any nonlinear factors are not significant for prediction. While there are other models that we could consider here, for brevity we focus on these two, and we leave further expansions for future work. 
where, LR is the load rating and SUP is the superstructure condition rating.

Given the existing models, we harvest from them the relevant factors that could describe the macro-level deterioration processes of a bridge, and we construct a System Dynamics (SD) diagram to illustrate the causal dynamic relationships between the different factors under consideration (Figure 19). As bridge condition and load rating describe the state of deterioration and state of capacity of a bridge at any point in time given the other factors, we set these two variables as the essential state variables of the engineering sub-model.

In this section we do not yet introduce the shared state variable that connects the engineering, social and economic modeling perspectives, but we simply build the engineering sub-model as seen from the perspective of the DOT and MPO decision makers.

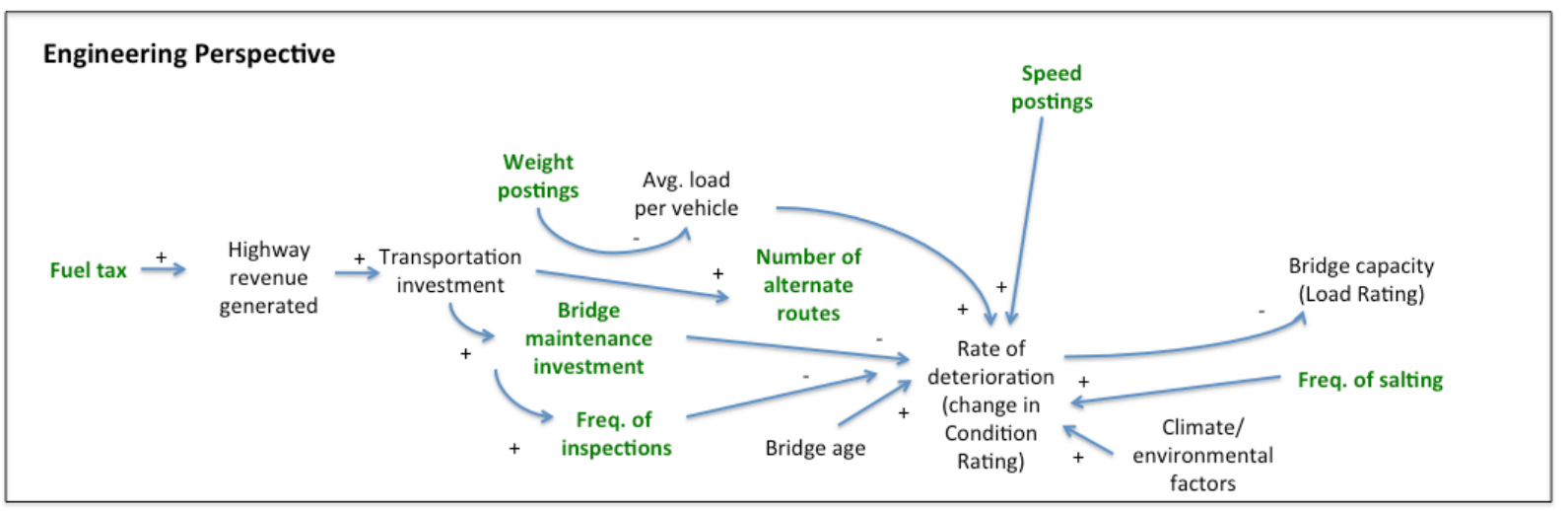

Figure 19: System dynamics (SD) diagram from the engineering perspective enables stakeholders to envision the dynamic relationships between the different factors under consideration

We now must consider whether the two statistical deterioration models discussed above are capable of answering the modeling questions of concern from the engineering perspective, and if not, we have to utilize the available information to construct a new model that will more adequately answer our questions. In this case study we would like to be able to assess how 
different long-term maintenance policies selected in period $i$ affect the superstructure condition and load ratings of the bridge at period $i+k(k=0,1, \ldots, T)$, and hence how they frame the extent of future maintenance options. Additionally, we would like to understand the impact of maintenance decisions on commercial and non-commercial users of the bridge now and in the future.

In order to facilitate this type of analysis, we can employ the Multiobjective Risk Impact Analysis Method (MRIAM) (Leach and Haimes, 1987), which combines the basic principles of multistage impact analysis (Gomide and Haimes, 1984) with the theory of extreme events and conditional risk expectations (Asbeck and Haimes, 1984), and enables decision makers to evaluate various risk functions and objectives at each step of the planning horizon. The general MRIAM formulation (Leach and Haimes, 1987) is given by Eq. 5-3.

$$
\begin{aligned}
& \bar{s}(k+1)=A \bar{s}(k)+B \bar{u}(k)+\bar{\omega}(k) \quad k=0, \ldots, T-1 \\
& \bar{y}(k)=C \bar{s}(k)+D \bar{u}(k)+\bar{v}(k)
\end{aligned}
$$

It is obvious that the general MRIAM formulation is equivalent to a Linear Time Invariant (LTI) system formulation (discussed in Chapter 4), so we adopt an LTI topology for our engineering sub-model. While there are certain detriments to using an LTI formulation, there are also certain benefits. In particular, LTI formulation guarantees the existence of closed form solutions, it implies constant parameters, and reflects stakeholders' concern with the time domain which is critically important in our problem.

\subsubsection{General engineering sub-model}

In order to construct an LTI representation of the engineering sub-model that would adequately answer our modeling questions, we conveniently utilize two existing engineering models discussed above (i.e., Chase et al. (1999) and Chase and Gáspár (2000)), which enable us to assess the changes in the bridge condition and load ratings, conditional on bridge age, changes in climate, and maintenance actions. We assume that from a macro-perspective these two models 
provide a good starting point to evaluate the macro-level changes in the superstructure condition and load rating over our planning horizon. We develop the following modeling construct:

$$
\begin{aligned}
& s_{\text {eng, } 1}(k+1)=a_{\text {eng }} \cdot s_{\text {eng, } 1}(k)+b_{\text {eng }} \cdot u_{\text {eng, } 1}(k)+c_{\text {eng }} \cdot u_{\text {eng, }, 2}(k)+d_{\text {eng }} \cdot u_{\text {eng, } 3}(k)+\omega_{\text {eng }}(k) \\
& s_{\text {eng, },}(k+1)=e_{\text {eng }} \cdot s_{\text {eng, }, 1}(k+1) \\
& y_{\text {eng, }}(k)=E\left[s_{\text {eng, } 1}(k)\right] \\
& y_{\text {eng }, 2}(k)=E\left[s_{\text {eng, }}(k) \mid \beta \leq \mu-\sigma\right]
\end{aligned}
$$

where,

- $k$ represents the bridge inspection period (inspections occur every two years hence $\Delta k=2$ years $)$

- $s_{\text {eng, } 1}(k)$ represents the superstructure condition rating (this variable ranges from $0-9$, with 9 representing a new bridge and 0 representing a non-operational bridge), and $s_{\text {eng, } 1}(0)$ is estimated for HRBT from the Chase et al. (1999) superstructure generalized linear regression model

- $s_{\text {eng, },}(k)$ represents the bridge load rating (in thousands of pounds), and $s_{\text {eng, } 2}(0)$ is estimated for HRBT from the Chase and Gáspár (2000) model

- $\quad u_{\text {eng, } 1}(k)$ represents the bridge maintenance investment (in \$)

- $u_{\text {eng, },}(k)$ represents the decision to implement a speed posting - a scalar variable indicating yes (1) or no (0)

- $u_{\text {eng,3 }}(k)$ represents the decision to implement a weight posting - a scalar variable indicating yes (1) or no (0)

- $\omega_{\text {eng }}(k)$ represents the normally distributed white noise with zero mean 
- $y_{\text {eng, },}(k)$ is the mean expected superstructure condition rating (We assume that $s_{\text {eng, },}(k)$ is normally distributed with mean $\mu$ and standard deviation $\sigma$ )

- $y_{\text {eng, } 2}(k)$ is the low range expected superstructure condition rating with partitioning $\beta$ taken at one standard deviation below the mean

We assume that from a traditional engineering perspective, DOT and MPO decision-makers have three major objectives (see Eq. 5-5), namely, maximizing the mean and low-range conditional expected bridge condition rating (or in this case superstructure condition rating) at the end of the planning horizon, which inevitably maximizes the mean and low-range conditional expected load rating at the end of the planning horizon as well, and minimizing the net present value (NPV) of maintenance investments at a discount rate $r \%$, if total investment in period $\mathrm{k}$ is constrained by budget $B_{k}$. We recognize that the NPV might not be the best metric for valuing long-term projects that might have considerable social and economic impacts on the region in question, and that certain metrics from the theory of welfare economics might be more suitable for assessing future worthiness of engineering projects (OECD, 2008). However, for this example we use the NPV as it is still widely used for transportation infrastructure projects. Furthermore, we recognize that in addition to these objectives, DOT and MPO decision makers have other less quantifiable and more politics-driven objectives, like for example satisfying the local or state politicians. While these objectives are not accounted for in Eq. 5-5, we address some of the less quantifiable issues in Chapter 6.

$$
\begin{aligned}
& \phi_{\text {eng, },}=\max _{u \in U_{\text {eng }}}\left(y_{\text {eng, },}(k)\right) \text { when } k=T \\
& \phi_{\text {eng, },}=\max _{u \in U_{\text {eng }}}\left(y_{\text {eng, }, 2}(k)\right) \text { when } k=T \\
& \phi_{\text {eng, } 3}=\min \left(\sum_{k=0}^{T} \frac{u_{\text {eng, } 1}(k)}{(1+r \%)^{k}}\right)
\end{aligned}
$$




$$
\begin{array}{lll}
0 \leq s_{\text {eng, } 1}(k) \leq 9 & \\
u_{\text {eng, } 1}(k) \leq B_{k} & & \\
u_{\text {eng, }, 2}(k)=0 & \text { or } & u_{\text {eng, },}(k)=1 \\
u_{\text {eng, }, 3}(k)=0 & \text { or } & u_{\text {eng, }, 3}(k)=1
\end{array}
$$

\subsubsection{Computing parameters of the engineering sub-model}

With regards to Eq. 5-4 in Section 5.5.1.1, coefficient $a_{\text {eng }}$ represents the linearized yearly change in the superstructure condition rating based on bridge age, amount of precipitation, frequency of deicing, and number of freeze-thaw cycles, and its initial estimate $\left(a_{\text {eng }}=0.989\right)$ was obtained for the HRBT (for years 2011 - 2035) from the superstructure condition rating model presented by Chase et al. (1999) (see Figure 20). ${ }^{2}$ This initial estimate suggests that in a 2-year period and under the assumptions of the Chase et al. (1999) model, the superstructure condition rating for the HRBT changes by approximately $1.1 \%$. As this estimate of the coefficient does not account for possibly extreme changes in the global and regional climate resulting in harsher winters and more precipitation, a sensitivity analysis was conducted by using the following values for this coefficient: $0.989,0.980,0.970$, representing respectively a $1.1 \%, 2 \%$ and $3 \%$ change in the superstructure condition rating over a 2-year period. Results presented in this Chapter reflect a $3 \%$ change in the superstructure condition rating over a 2 -year period.

\footnotetext{
2 The superstructure condition rating for HRBT in year 2011 is approximately $\mathrm{N}\left(5.86,0.154^{2}\right)$, and in year 2035 it is approximately N $\left(5.12,0.154^{2}\right)$. These values were computed by Zhenyu Guo for the NSF project Adaptive Systems-Based Prioritization of Bridge System Maintenance: Integrated Modeling of Technical, Socioeconomic, and Normative Dimensions.
} 


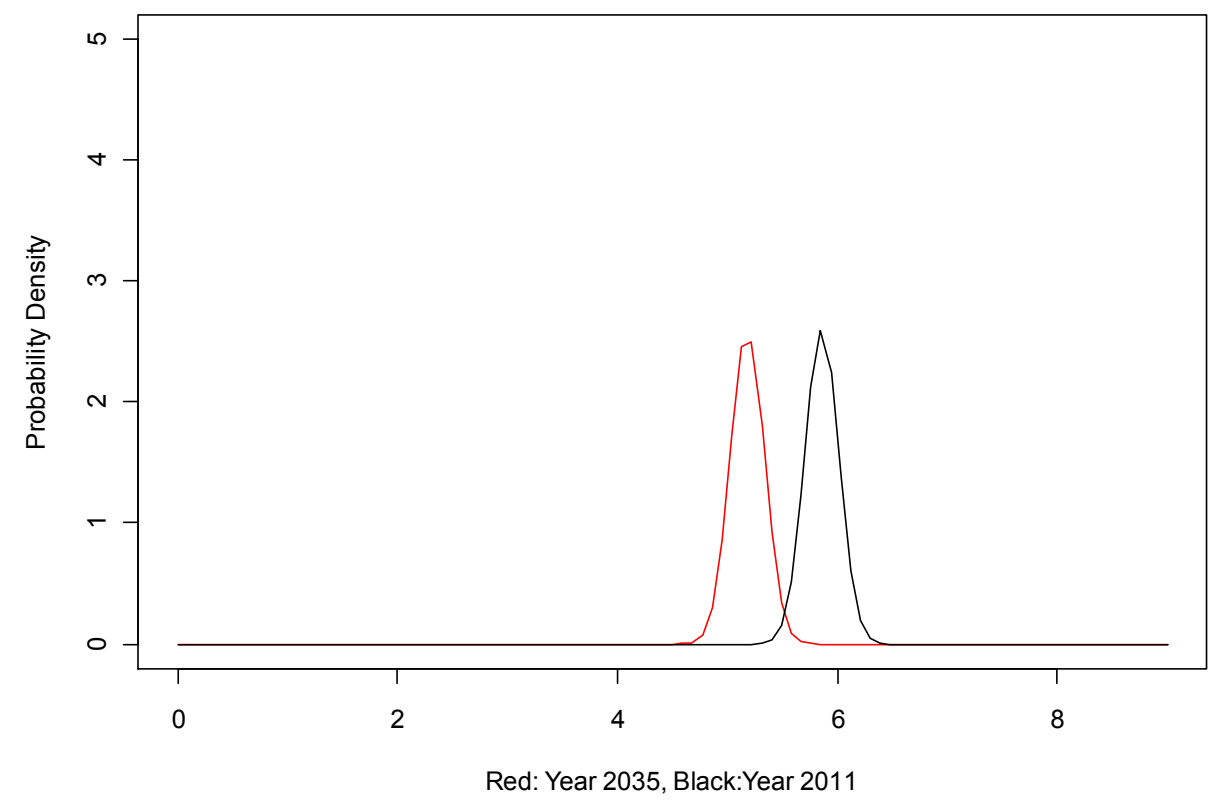

Figure 20: Change in HRBT superstructure condition rating from 2011 to 2035 based on model from Chase et al. (1999) (see footnote 2)

Parameter $b_{\text {eng }}$ denotes the change in the bridge condition rating based on the maintenance investment made during the previous planning period of two years. In other words, it estimates the effectiveness of maintenance activities. Ideally this parameter would be identified by conducting a time-series analysis comparing the changes in superstructure condition rating for a particular bridge to actual maintenance expenditures for the same bridge. However, bridge maintenance investments for individual bridges are not recorded in a standard fashion, and many state DOTs do not track in house spending adequately. The Long Term Bridge Performance Program (Friedland et al., 2007) will provide a framework through which such data will be collected, but as of now accurate data is unavailable. The goal of the program will be to collect and develop "information and routines that quantify the effectiveness of various maintenance, repair, and rehabilitation strategies" (Friedland et al., 2007). Currently through the Federal Highway Administration's (FHWA's) Safe Accountable Flexible Efficient Transportation Equity Act (SAFETEA) (US DoT SAFETEA-LU), FHWA collects and disseminates data on infrastructure apportionments of Federal-aid highway program funds to states for various 
transportation programs, including highway bridges. While they provide information on federal annual apportionments for highway bridges in Virginia (see Funding Tables of the SAFETEA$\mathrm{LU}$ ), they do not provide information on the state and local level spending patterns for particular bridge projects. Additionally, the apportionments for the highway bridge program do not equal actual expenditures for the highway bridge maintenance, hence at its present state we do not utilize this data to compute parameter $b_{\text {eng }}$ for the time period of interest. Instead, we acknowledge that this parameter would be dependent on many factors, including the type and size of the bridge, the timing of maintenance and inspection periods, and many others, and for this illustrative example we make a simplifying assumption that a $\$ 10,000,000$ maintenance investment will increase the superstructure condition rating of a bridge by 1 point, hence $b_{\text {eng }}=$ $10^{-7}$. In the future, with the addition of more accurate data, this parameter could be reassessed by following existing approaches in the pavement maintenance literature (Ramaswamy and BenAkiva, 1990; Mouaket and Sinha, 1991; Fwa and Sinha, 1987; Madanat and Mishilani, 1995; Labi and Sinha, 2003).

Parameter $\mathrm{c}_{\text {eng }}$ reflects the impact of a speed posting on the superstructure condition rating. If the DOT decision makers determine that a speed posting is not warranted, then $\mathrm{c}_{\mathrm{eng}}=0$. If a speed posting is put in place, then $\mathrm{c}_{\mathrm{eng}}>0$, and the actual value would depend on the actual speed limit. Parameter $d_{\text {eng }}$ reflects the impact of a weight posting on the superstructure condition rating. If the DOT decision makers determine that a weight posting is not warranted, then $d_{\text {eng }}=0$. If a weight posting is put in place, then $\mathrm{d}_{\text {eng }}>0$, and the actual value would depend on the actual weight limit. The identification of $c_{\text {eng }}$ and $d_{\text {eng }}$ is not a trivial task, as the effects of speed and weight postings are dependent on many factors. The identification of these parameters could be conducted by performing time-series analysis to compare superstructure condition ratings on bridges that are comparable in structure, size and traffic loadings, and some of which require speed and/or weight postings and some of which do not. This is left for future work as it is outside of the scope of this dissertation.

Parameter $\mathrm{e}_{\mathrm{eng}}=4.37$ relates the bridge condition rating to the bridge load capacity, and the value was adopted from Chase and Gáspár (2000). Additionally, for Eq. 5-4 we assume that $\omega(k)$ is normally distributed and purely random with mean 0 and variance 0.01 . We also assume 
that the initial state $s_{\text {eng, } 1}(0)$ for HRBT is normally distributed, and that $s_{\text {eng, } 1}(0)=5.86$ (obtained from the Chase et al. (1999) model for year 2011 - see footnote 2).

\subsubsection{Constructing the social sub-model}

From the perspective of non-commercial bridge users (i.e., the social modeling perspective), untimely and insufficient bridge maintenance is of concern only to the extent that it affects the ability of individuals to travel over the bridge of interest. The inability of non-commercial travelers to travel over the bridge can result in many ripple effects, including effects on workforce commute and earnings, changes in real-estate prices, future economic development, and many others which have been studied in existing literature (see Chapter 2). There are many factors responsible for determining travel habits and patterns of non-commercial bridge users (Dong et al., 2006; Ettema et al., 1995; Fujii et al., 1997; Hamed and Mannering, 1993; Hirsch et al., 1985, 1986; Kitamura, 1995, 1996; Kitamura et al., 1996), among which are natural changes in the general population, impact of regional economy on work and non-work car trips, personal preferences and constraints, as well as the impact of travel time and cost of travel (Figure 21). Numerous quantitative and qualitative approaches have been developed to study the effects of some of these factors on travel patterns (see NCHRP (2001) for details). However, for the purpose of this example, we focus on quantifying only the total number of non-commercial bridge users, and the average travel time cost. The total number of non-commercial bridge users serves as a surrogate measure for social accessibility, which in reality is very difficult to estimate.

As in the engineering sub-model, we adopt an LTI representation that will allow us to compare, over the planning horizon of interest, the anticipated changes in non-commercial traffic with anticipated changes in the structural condition of the bridge, caused by selected maintenance decisions. We recognize that the rate of changes occurring in the three sub-systems will likely not be the same. In other words, changes occurring in the engineering sub-system will likely take a longer time to become apparent. However, we assume that by capturing macroeffects every two years, we sufficiently address this issue. We furthermore acknowledge that while the micro-changes in the social and economic sub-systems are not linear, in our approach we consider the general changes in population and economy to be sufficiently linear over a 
longer period of time.

In this section we do not yet introduce the shared state variable that connects the engineering, social and economic modeling perspectives, but we simply build the social sub-model as seen from the perspective of the non-commercial bridge users.

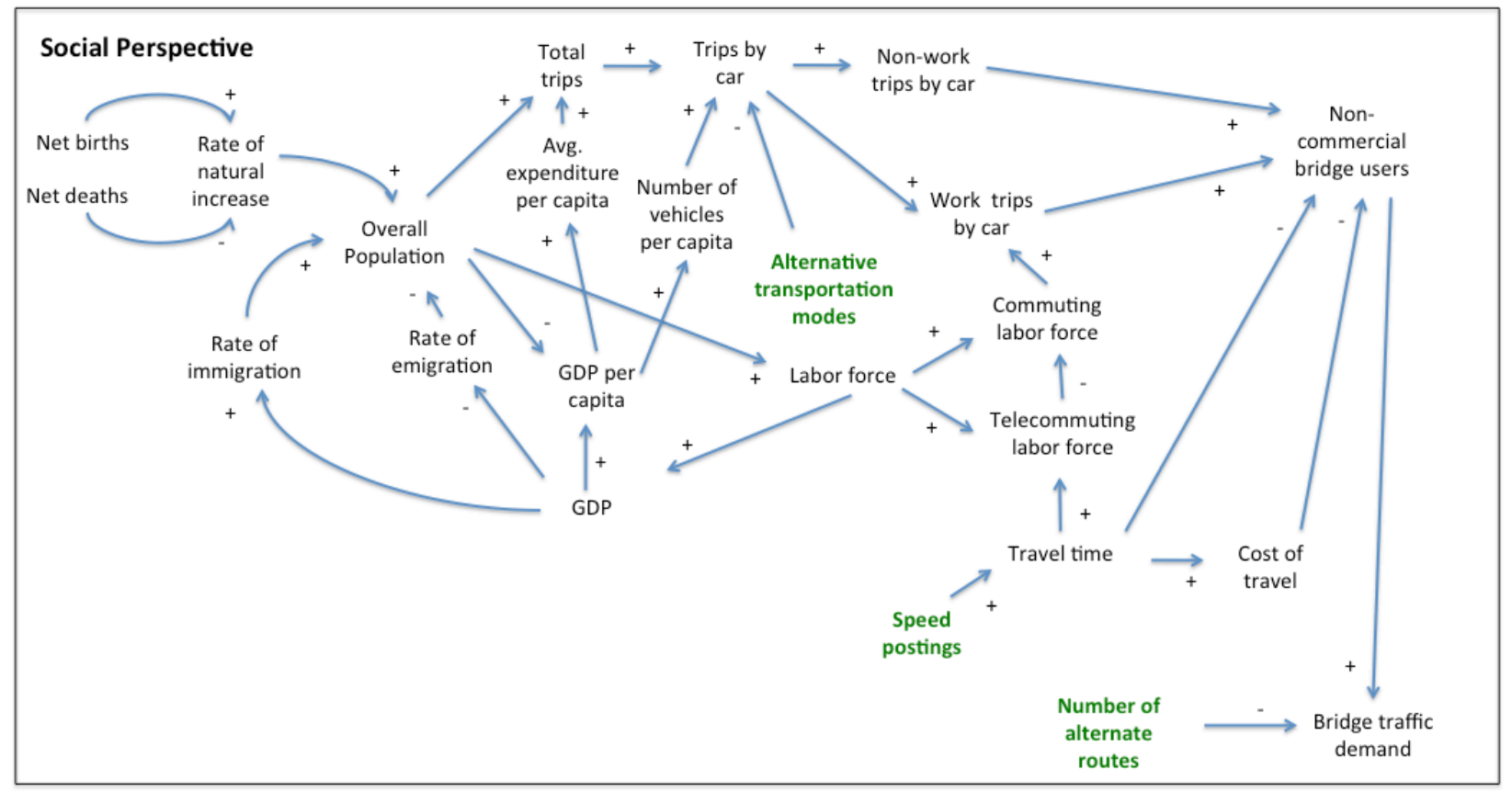

Figure 21: SD diagram from the social perspective

\subsubsection{General social sub-model}

We select one essential state variable to represent the state of the system at any point in time, namely, the average annual daily non-commercial traffic that can safely cross over the bridge. This number depends on the size and growth patterns of the overall population, and it is impacted by decisions made in the social sub-system, which are implicitly accounted for in the social sub-model (e.g., decision to travel for work or non-work reasons, decision to utilize the HRBT) as well as the decisions made in the engineering sub-system (e.g., speed postings, weight postings). Our LTI representation of the social sub-model is given in Eq. 5-6. 
$s_{s o c, 1}(k+1)=a_{s o c} \cdot s_{s o c, 1}(k)-b_{s o c} \cdot u_{s o c, 1}(k) \cdot s_{s o c, 1}(k)+\omega_{s o c}(k)$

$y_{s o c, 1}(k)=E\left[s_{s o c, 1}(k)\right]$

where,

- $k$ represents the discrete assessment point ( $\Delta k=2$ years $)$

- $s_{s o c, 1}(k)$ represents the normally distributed average annual daily non-commercial traffic over the bridge (We are making an assumption that each trip is conducted by a single individual, thus this state variable reflects the size of the population that is dependent on the bridge for work-commuting, social, cultural, and personal activities.)

- $u_{s o c, 1}(k)$ represents the input into the model from the engineering perspective that indicates a speed posting flag ( 0 if no speed posting was introduced in period $k$, or 1 if a speed posting was put into place in period $k$ )

- $\omega_{\text {soc }}(k)$ represents the normally distributed white noise with zero mean

- $y_{s o c, 1}(k)$ is the mean expected average annual daily non-commercial traffic over HRBT

The goal of the stakeholders representing the social sub-system is to perform their daily work and non-work (discretionary) activities with as few disruptions as possible, and at a lowest possible cost. Hence, from the social perspective stakeholders wish to maximize the average annual daily non-commercial traffic over the bridge at any period of time (as this number serves as a surrogate measure of the ability of individuals to conduct their daily activities), as well as minimize the average total travel cost per non-commercial traveler $\left(\mathrm{TC}_{\mathrm{soc}}\right)$. We assume that the decisions performed by the non-commercial stakeholders are implicitly accounted for in the social sub-model. These decisions refer to making a choice to conduct or not conduct the trip and making the choice to conduct or not conduct the trip over the HRBT versus an alternative. The objectives of the stakeholders from the social perspective are expressed in Eq. 5-7. 


$$
\begin{aligned}
& \phi_{s o c, 1}=\max _{u \in U_{s o c}}\left(y_{s o c, 1}(k)\right) \quad \forall k=0, \ldots, T \\
& \phi_{s o c, 2}=\min _{u \in U_{s o c}}\left(\sum_{k=0}^{T} T C_{s o c}(k)\right)
\end{aligned}
$$

s.t.

$T C_{s o c}=T T C_{s o c}+S I M C_{s o c}$

$$
T T C_{s o c}=\frac{1000}{A E S} \cdot T T V A L_{s o c}
$$

In Eq. 5-7, $\mathrm{TC}_{\mathrm{soc}}$ represents the average total cost per non-commercial traveler (in $\$$ per thousand vehicle miles). $\mathrm{TTC}_{\text {soc }}$ represents the average travel time cost (in $\$$ per thousand vehicle miles). TTC $_{\text {soc }}$ is a metric commonly used in the Federal Highway Administration's HERST-ST Highway Economic Requirements System (US DOT - HERST-ST, 2002, 2005). Although the HERS-ST model has many component costs that are used to estimate $\mathrm{TTC}_{\mathrm{soc}}$, we use a simplified version of this model to obtain a general estimate of direct costs to the traveler. AES represents the average effective speed of a vehicle on the bridge. We assume that the introduction of a speed limit slightly reduces the average effective speed of the traffic on the bridge, and thus increases the $\mathrm{TTC}_{\mathrm{soc}}$. For illustrative purpose in this example we assume that the introduction of a speed limit reduces the overall speed flow by 1 mile per hour. TTVAL ${ }_{\text {soc }}$ represents the average value of time (in \$) per occupant of a non-commercial vehicle. Table 5-27 of (US DOT - HERS ST, 2005) provides values (in 1995 dollars) of one hour of travel time for commercial and noncommercial users traveling in different types of vehicles. In addition to these costs, we introduce an additional component of the total cost, namely, the shared infrastructure maintenance cost $\mathrm{SIMC}_{\mathrm{soc}}$ (in $\$$ per thousand vehicle miles). This cost component reflects whether the noncommercial users are participating in infrastructure maintenance costs through bridge usage tolls, increased gas taxes, additional maintenance taxes, or other innovative funding mechanisms. Essentially, $\mathrm{SIMC}_{\text {soc }}$ (and as we will see in Section 5.5.3, $\mathrm{SIMC}_{\text {econ }}$ ) could be used to increase the overall maintenance budget for bridge maintenance projects $\left(\mathrm{u}_{\mathrm{eng}, 1}\right)$. In this example we assume 
that at $\mathrm{k}=0, \mathrm{SIMC}_{\mathrm{soc}}=0$, but that this value could be altered during the collaborative coordination phase, as will be discussed in Section 5.6.

\subsubsection{Computing parameters of the social sub-model}

In the social sub-model we make a simplifying assumption that the growth of the noncommercial bridge traffic demand is proportional to the population growth rate, hence we estimate parameter $a_{\text {soc }}$ based on the U.S. Census annual population estimates in the NorfolkVirginia Beach-Newport News metropolitan area from 1999 to 2009 (U.S. Census - Population Estimates). The average yearly annual population increase in the Hampton Roads Area is $0.76 \%$. We assume that this rate will remain constant for the planning horizon of interest, and assuming no compounding effects, the average population increase over a two-year period $(k)$ is then $1.52 \%$, thus making $a_{\text {soc }}=1.0152$.

Additionally, we assume that a speed posting placed on the HRBT will increase the travel time and hence the travel cost for non-commercial users. As some of the non-commercial trips taken in the region are not work-based, we assume that a small percentage of non-commercial users will choose to not travel if the cost of travel increases, i.e., if the speed posting is put in place. Thus, we introduce a reduction in the number of non-commercial travelers at time $k+1$ if a speed posting is put in place at time $k$. For illustrative purpose we set $b_{\text {soc }}=0.25 \%$, indicating that when a speed posting is introduced, $0.25 \%$ of existing travelers will choose not to travel, thus reducing $\mathrm{S}_{\mathrm{soc}, 1}$.

Given the lack of the exact data on the distribution of vehicle types that cross the HRBT at any point in time, we assume that the medium car can serve as a representative non-commercial vehicle for the HRBT, and we use $\mathrm{TTVAL}_{\mathrm{soc}}=\$ 23.68$ for the average value of one-hour of travel time per vehicle (1995 values from US DOT - HERS ST (2005) were adjusted for inflation to 2012 dollars). 


\subsubsection{Constructing the economic sub-model}

From the perspective of commercial bridge users (i.e., the economic modeling perspective), untimely and insufficient bridge maintenance is of concern only to the extent that it affects the ability of commercial vehicles (primarily freight carriers) to travel over the bridge of interest. The inability of commercial travelers to travel over the bridge can cause disruptions to manufacturing production schedules and sales patterns. There are many factors responsible for determining travel patterns of commercial bridge users, among which are changes in the size of the regional industry, legally allowable weight loads, and others (Figure 22). For the purpose of this example, we focus on quantifying the total number of commercial travelers, the average annual daily commodity load transported, and the average travel time cost. The total number of commercial travelers serves as a surrogate measure for economic accessibility, which in reality is very difficult to estimate. In this section we do not yet introduce the shared state variable that connects the engineering, social and economic modeling perspectives, but we simply build the economic sub-model as seen from the perspective of the commercial bridge users.

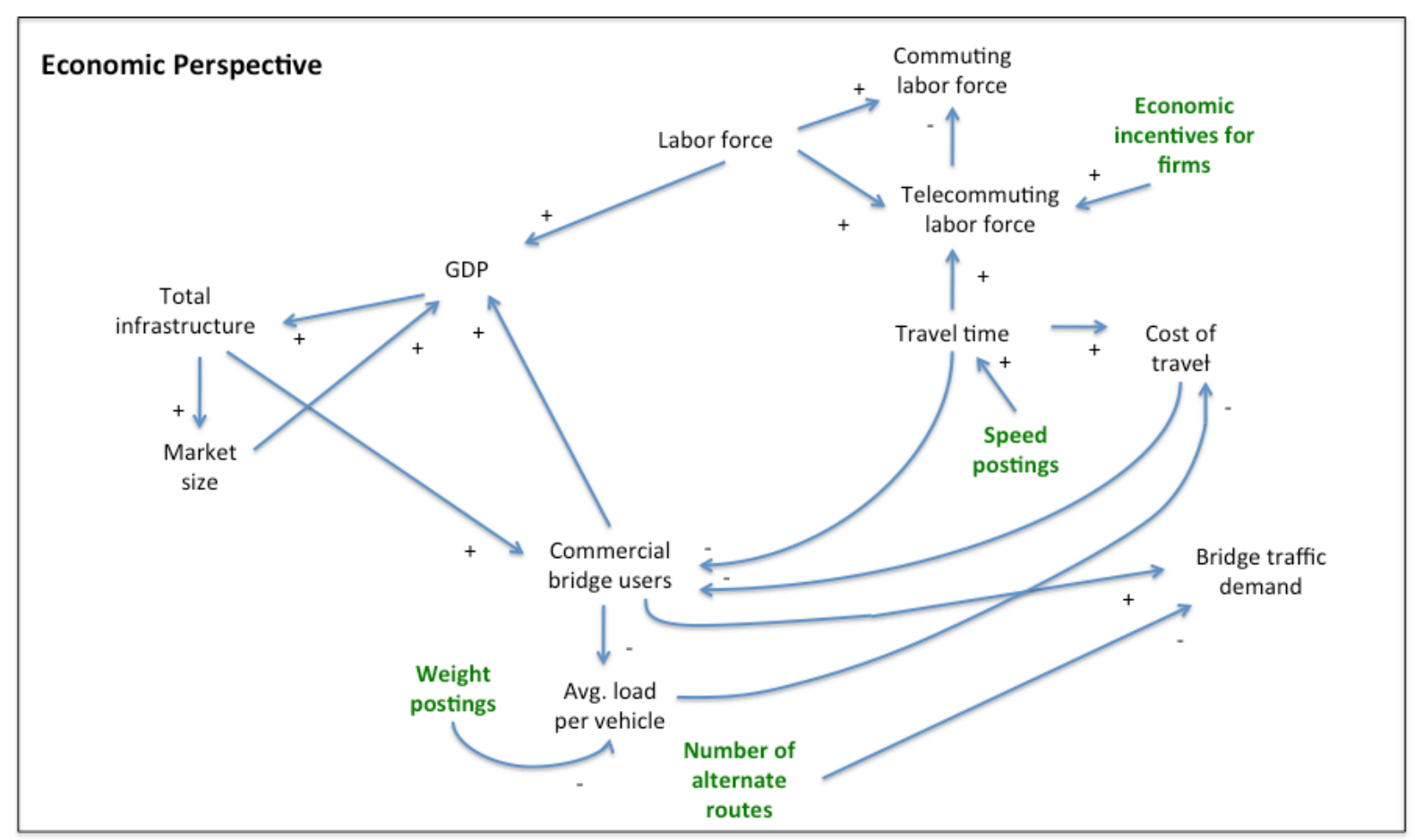

Figure 22: SD diagram from the economic perspective 


\subsubsection{General economic sub-model}

We again adopt an LTI representation that will allow us, over time, to compare the anticipated changes in commercial traffic with anticipated changes in the structural condition of the bridge, resulting from selected maintenance decisions. We select two essential state variables to represent the state of the system at any point in time, namely, the average annual daily commercial traffic that can safely cross over the bridge, and the average annual daily commodity load transported over the bridge. The LTI representation of the economic sub-system is given in Eq. 5-8.

$$
\begin{aligned}
& s_{\text {econ }, 1}(k+1)=a_{\text {econ }} \cdot s_{\text {econ }, 1}(k)+\omega_{\text {econ }, 1}(k) \\
& s_{\text {econ }, 2}(k+1)=b_{\text {econ }} \cdot\left(1-c_{\text {econ }} \cdot u_{\text {econ }, 1}(k)\right) \cdot s_{\text {econ }, 1}(k+1)+\omega_{\text {econ }, 2}(k) \\
& y_{\text {econ }, 1}(k)=E\left[s_{\text {econ }, 1}(k)\right]
\end{aligned}
$$

where,

- $k$ represents the discrete assessment point $(\Delta k=2$ years $)$

- $s_{\text {econ, } 1}(k)$ represents the normally distributed average annual daily commercial traffic over the bridge

- $s_{\text {econ,2 }}(k)$ represents the average annual daily commodity load transported over the bridge

- $u_{e c o n, 1}(k)$ represents the input into the model that indicates a weight posting flag ( 0 if no weight posting was introduced in period $k$, or 1 if a weight posting was put into place in period $k$ )

- $\omega_{\text {econ }, i}(k)$ represents the normally distributed white noise with zero mean

- $y_{\text {econ, } 1}(k)$ is the mean expected average annual daily commercial traffic over HRBT 
The goal of the stakeholders representing the economic sub-system is to perform their scheduled commercial activities (i.e., delivery of intermediate supplies and finished goods, manufacturing processes, etc.) with as few disruptions as possible, and at a lowest possible cost. Hence, from the social perspective stakeholders wish to maximize the average annual daily commercial traffic over the bridge at any period of time (as this number serves as a surrogate measure of the ability of companies, industrial or service sectors to conduct their business), as well as minimize the average total travel cost per commercial traveler $\left(\mathrm{TC}_{\text {econ }}\right)$. We assume that the decisions performed by the commercial stakeholders are implicitly accounted for in the economic sub-model. These decisions refer to making a choice to conduct or not conduct the trip and making the choice to conduct or not conduct the trip over the HRBT versus an alternative route. The objectives of the stakeholders from the economic perspective are expressed in Eq. 5-9.

$$
\begin{aligned}
& \phi_{\text {econ }, 1}=\max _{u \in U_{\text {econ }}}\left(y_{\text {econ }, 1}(k)\right) \quad \forall k=0, \ldots, T \\
& \phi_{\text {econ }, 2}=\min _{u \in U_{\text {econ }}}\left(\sum_{k=0}^{T} T C_{\text {econ }}(k)\right)
\end{aligned}
$$

s.t.

$$
\begin{aligned}
& T C_{\text {econ }}=T T C_{\text {econ }}+S I M C_{\text {econ }} \\
& T T C_{\text {econ }}=\frac{1000}{A E S} \cdot T T V A L_{\text {econ }}
\end{aligned}
$$

In Eq. 5-9, $\mathrm{TC}_{\mathrm{econ}}$ represents the total cost per commercial traveler (in $\$$ per thousand vehicle miles). $\mathrm{TTC}_{\text {econ }}$ represents the average travel time cost (in $\$$ per thousand vehicle miles). $\mathrm{TTC}_{\text {econ }}$ is a metric commonly used in the Federal Highway Administration's HERST-ST Highway Economic Requirements System (US DOT - HERST-ST, 2002, 2005). Although the HERS-ST model has many component costs that are used to estimate $\mathrm{TTC}_{\text {econ, }}$, we use a simplified version of this model to obtain a general estimate of direct costs to the traveler. AES represents the average effective speed of a vehicle on the bridge. TTVAL $L_{\text {econ }}$ represents the average value of 
time (in \$) per occupant of a commercial vehicle. Table 5-27 of (US DOT - HERS ST, 2005) provides values of one hour of travel time for commercial and non-commercial users traveling in different types of vehicles. These values are shown in 1995 dollars. Given the lack of the exact data on the distribution of vehicle types that cross the HRBT at any point in time, we assume that a 5-axle comb. truck can serve as a representative commercial vehicle for the HRBT, and we use for the average value of one-hour of travel time per vehicle TTVAL $_{\text {econ }}=\$ 37.95(1995$ values from US DOT - HERS ST (2005) were adjusted for inflation).

\subsubsection{Computing parameters of the economic sub-model}

In Eq. 5-8 parameters $a_{e c o n}$ and $b_{e c o n}$ are estimated from a regional freight study conducted by the Hampton Roads Regional Planning District Commission (2007). This report provides estimates of the inbound and outbound freight movements (in terms of tonnage and dollar value) from 2004 to 2035. Within these three decades, freight traffic in the Hampton Roads Area was expected to grow by approximately $4.88 \%$ a year, based on tonnage. We assume that the yearly growth rate of freight traffic over the bridge of interest is the same as the growth rate for all commercial traffic passing through the area, hence assuming no compounding effects, for a twoyear period this growth rate is $9.76 \%$. Then, $a_{\text {econ }}=1.0976$. Parameter $b_{\text {econ }}=66,055$ represents the average commodity load (in pounds) transported on a single commercial trip, and it is obtained from the same study.

We assume that a weight posting placed on the HRBT will decrease the average load that can be transported by a single truck. We introduce a reduction in the average commodity load transported on a single commercial trip at time $k+1$ if a weight posting is put in place at time $k$. For illustrative purposes we set $\mathrm{c}_{\mathrm{econ}}=0.25 \%$, indicating that when a weight posting is introduced, the average commodity load transported on a single commercial trip is reduced by $0.25 \%$. 


\subsubsection{Harmonizing the three sub-models into a meta-model through the introduction of a shared state variable}

Diverse stakeholders (representing the engineering, social, and economic perspectives) might have different views of what the "real problem" associated with insufficient and untimely bridge maintenance is, and their interpretation depends on the limited context within which they operate, and on the time frame that is of interest to them (and that might differ from one perspective to the next). Ultimately, we would like to integrate the three modeling perspectives into a single system of systems meta-model that will provide us with a more holistic view of the problem, and that will enable different stakeholders to: (i) better envision any shared and conflicting interests, concerns and constraints, and (ii) better coordinate their decentralized yet interdependent decisions. The question then becomes of how the three different perspectives can be harmonized so that the knowledge from one perspective can be shared with the other perspectives, for the purpose of selecting long-term bridge maintenance policies that are cognizant of and responsive to emergent changes occurring in different sub-systems.

As described in Chapter 4, at this stage we introduce a shared state variable that is of concern to all stakeholders (although it might not be immediately obvious to all stakeholders). The engineering, social and economic SD diagrams (Figure 19, Figure 21, and Figure 22) are integrated into a single SD diagram (Figure 23) to identify factors that tie the three modeling perspectives together. In determining what connects the three modeling perspectives we note that the number of commercial and non-commercial users together represent the total traffic demand placed on the bridge. From the engineering perspective the bridge load capacity determines the maximum live load that a bridge can safely carry. Hence, we introduce a physical measure of the total number of vehicles that can utilize the bridge in a certain period of time, which we term bridge traffic capacity. This variable describes the state of the overall system at some point in time, and it relates the number of commercial and non-commercial users of the bridge with the structural characteristics of the bridge and its load rating. This shared state variable essentially gives an indication of the additional traffic capacity that may be available on the bridge at a certain period of time, given current and projected commercial and non-commercial demand, and physical changes of the bridge structure. 
Through the intrinsic meta-modeling collaborative coordination process, described in Chapter 4, these diverse and decentralized stakeholders can collaboratively combine their aggregate and cross-disciplinary knowledge to assess the impacts on the shared state variable from decisions and processes associated with different perspectives, in order to achieve some globally acceptable range of values of the shared state variables. We recall that in Chapter 4 we defined intrinsic meta-modeling coordination as an iterative and collaborative process of adjusting actions in interdependent sub-systems (e.g., engineering, social, economic) by manipulating individual sub-system's decisions and constraints in response to observable or measurable changes in shared state variables, in order to achieve more satisficing system of systems solutions. For a problem involving the maintenance of the bridge infrastructure, these changes to the sub-systems' decision spaces might include: (i) innovative funding mechanisms (e.g., private-public partnerships, tolls, usage taxes) that would require some or all infrastructure users to participate in infrastructure maintenance financing $\left(\mathrm{SIMC}_{\mathrm{soc}}\right.$ and/or $\mathrm{SIMC}_{\text {econ }}$ ); (ii) incentives and/or commitment devices that would encourage regional industries to support employee telecommuting or to participate in maintenance funding by paying infrastructure usage fees for trucks that exceed a certain weight. These options are discussed in more detail in Chapter 6.

It is important to note that while the interpretation of the shared state variable might be different from different perspectives (see Eqs. 5-10, 5-12, 5-13), ultimately, in order for the HRBT to remain operational and sustainable over the entire planning horizon, the stakeholders must ensure that the additional traffic capacity is not negative. Otherwise there exists a risk that the load exerted on the bridge might become greater than the actual physical capacity of the bridge. In other words, stakeholders must agree on some range of values of a shared state variable that would be acceptable from all perspectives over the entire planning horizon. 


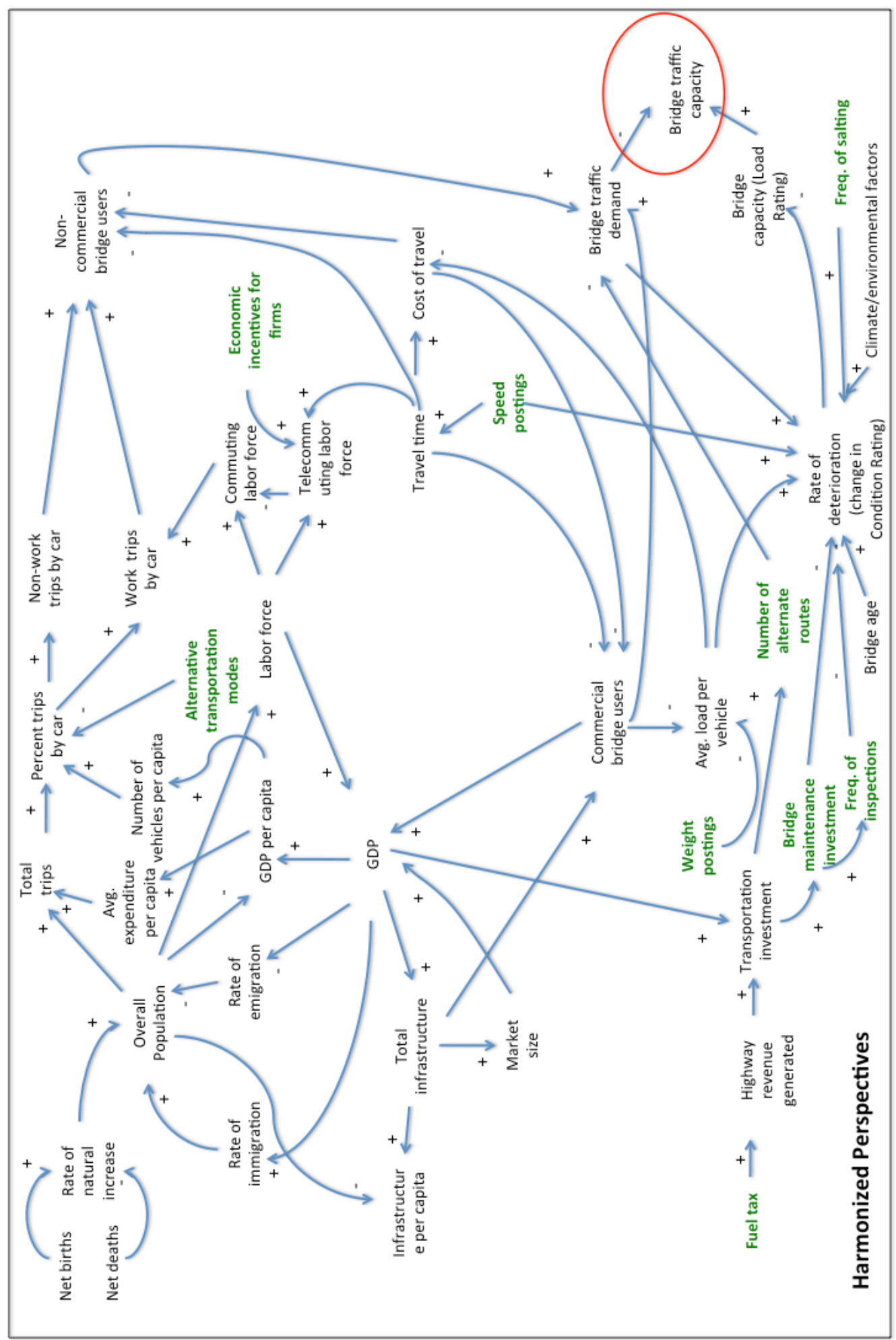

Figure 23: SD diagram for integrated considerations. The shared state variable connecting the three modeling perspectives (bridge traffic capacity) is circled. 
We now have to harmonize the three sub-models into a single meta-model through the bridge traffic capacity. We do this by introducing coupling functions for each sub-system. These coupling functions link bridge traffic capacity to a subset of the unshared variables in each subsystem. For example, in the engineering sub-model, we link bridge traffic capacity to bridge load rating, as indicated in Eq. 5-10.

$s_{\text {eng, } 3}(k+1)=f_{\text {eng }} \cdot s_{\text {eng, } 2}(k+1)$

In this linking function, parameter $\mathrm{f}_{\text {eng }}=5.036$ converts bridge load carrying capacity $\left(\mathrm{s}_{\text {eng, }, 2}\right)$ to the number of vehicles that can safely cross the HRBT in a given day. In this illustrative example Eq. 5-11 was used to calculate this parameter for HRBT.

$$
\begin{aligned}
f_{\text {eng }}= & \frac{\text { number bridge spans }}{\text { weighted average vehicle weight }[\mathrm{lbs}]} \cdot \\
& \cdot \frac{\text { average traffic flow speed }[\mathrm{mph}]}{\text { length of bridge }[\text { miles }]} \cdot 24 \text { hours } \\
= & \frac{123}{5,861.65 \mathrm{lbs}} \cdot \frac{35 \mathrm{mph}}{3.5 \mathrm{miles}} \cdot 24 \text { hours } \\
= & 0.2098385 \mathrm{lbs}^{-1} \cdot 10 \mathrm{hr}^{-1} \cdot 24 \mathrm{hr} \\
= & 5.036 \mathrm{lbs}^{-1}
\end{aligned}
$$

We acknowledge that Eq. 5-11 is simplistic and does not account for distribution of loads per axle of a moving vehicle, however, we argue that it is sufficient for illustrating the effectiveness of the intrinsic meta-modeling approach. 
Similarly we augment the social and economic sub-models by adding a coupling function to each (see Eqs. 5-12 and 5-13). We note that from the social and economic perspective the bridge traffic capacity can be expressed as the sum of all commercial and non-commercial travelers, in addition to the yet unutilized additional capacity.

$s_{s o c, 2}(k+1)=s_{s o c, 1}(k+1)+u_{s o c, 2}(k+1)+$ additional capacity

where, $s_{s o c, 2}(k)$ represents the bridge traffic capacity from the social perspective (i.e., the maximum number of average annual daily vehicles that can cross the bridge), and input $u_{s o c, 2}(k)$ represents the average annual daily commercial traffic over the bridge (this is the state variable $s_{\text {econ, } 1}(k+1)$ in the economic sub-model).

$s_{\text {econ, }, 3}(k+1)=s_{\text {econ, }}(k+1)+u_{\text {econ }, 2}(k+1)+$ additional capacity

where, $s_{\text {econ }, 3}(k)$ represents the bridge traffic capacity (i.e., the maximum number of average annual daily vehicles that can cross the bridge), and input $u_{\text {econ, }}(k)$ represents the average annual daily non-commercial traffic over the bridge (this is a state variable $s_{s o c, 1}(k+1)$ in the social sub-model). Now that we have introduced coupling functions that connect our three submodels through a shared state variable, we can develop a single harmonizing meta-model (Figure 24). 


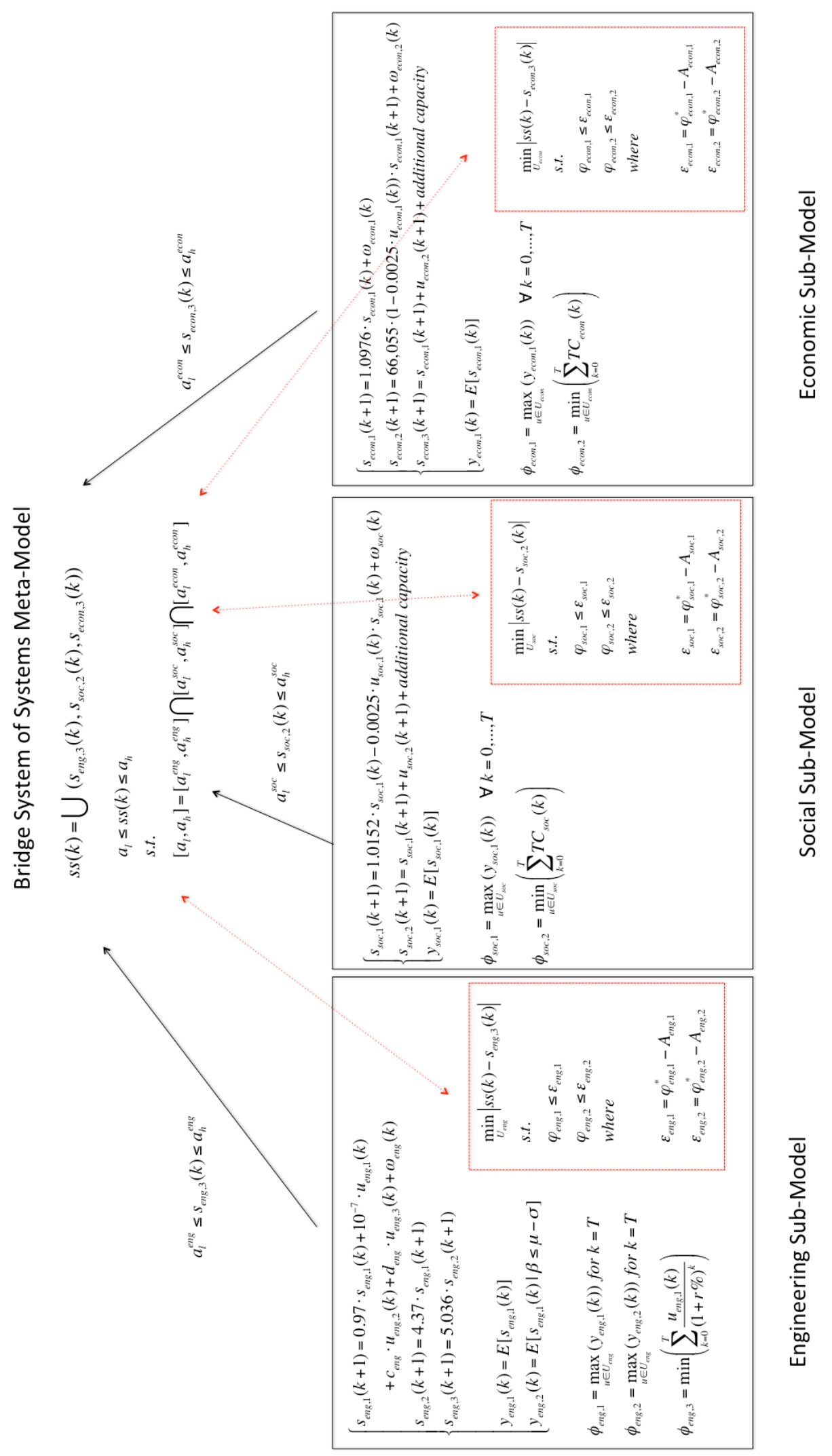

Figure 24: Bridge SoS Meta-Model 


\subsection{Traditional solution without collaborative meta-model coordination}

The decentralized decision making process that takes place in a bridge system of systems often leads to global solutions that are not acceptable to all stakeholders -- since decisions made in interdependent sub-systems affect shared state variables, then an uncoordinated decision making process could result in levels of shared state variables that are not acceptable to all involved parties over a longer planning horizon. In a traditional engineering approach, social and economic stakeholders have little to no say when it comes to selecting maintenance options for bridges, thus for this first case, we assume that only engineering sub-system stakeholders are involved in making a decision about the best maintenance alternative. As an example, we consider which of the illustrative maintenance policies, presented in Table 3, would be most acceptable to DOT and MPO decision makers, assuming that they have no knowledge of the subsystem interdependencies. If we only consider the traditional engineering objectives discussed in Section 5.5.1.1, and we assume an investment discount rate of $10 \%$, we come to a conclusion that Policy 1 from Table 3 (end loaded policy in which maintenance investment is not made until the end of the planning horizon) is most preferred from a traditional engineering perspective, as it results in highest end-of-planning-period mean and conditional expected superstructure condition and load ratings, and the lowest NPV (Figure 25).

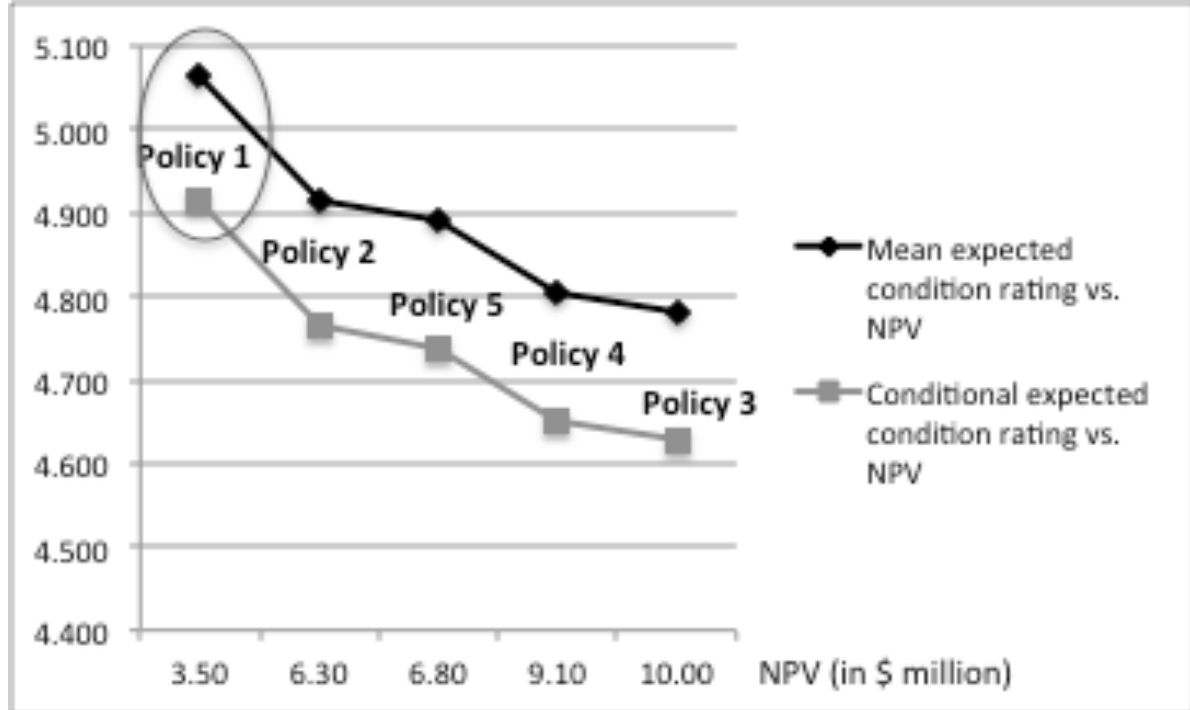

Figure 25: End-of planning horizon superstructure condition rating versus NPV for five maintenance policies (discounted at $10 \%$ ) 


\subsection{Holistic solutions with collaborative meta-model coordination}

If we now consider that DOT and MPO decision makers have knowledge of the shared state variable (bridge traffic capacity), and thus have some insight into the sub-system interdependencies, then we can assume that in addition to the end-of-planning-horizon bridge condition and load rating, they might also be interested in two other performance measures. The first of those is the consistency of condition ratings over the entire planning horizon, where consistency is defined as the ratio between the maximum and minimum superstructure condition rating achievable over the planning horizon under a specific policy (Figure 26). The second is a measure of the available additional traffic capacity resulting from a specific maintenance policy, aggregated over the entire planning horizon (Figure 27).

Assuming no speed or weight postings have been issued for HRBT, we assess the values of the bridge traffic capacity resulting from the five maintenance policies at each period $k$, where $k$ $=0,1, \ldots \mathrm{T}$. At each period $k$ the bridge traffic capacity is compared to the traffic demand, and an additional traffic capacity value is recorded. A positive value indicates that the bridge is able to support additional traffic. A negative capacity indicates that the bridge is unable to support projected demand. As can be seen from Figure 27, all of the five alternative maintenance policies result in negative additional traffic capacity aggregated over the entire planning horizon.

Recall that the objectives of the stakeholders from the social and economic perspectives are to maximize the number of commercial and non-commercial vehicles traveling over the bridge (i.e., to maximize social and economic accessibility), and to minimize the overall cost of travel. As negative expected bridge traffic capacity reduces the number of vehicles that can safely travel over the bridge, causes delays, and thus increases $\mathrm{TTC}_{\mathrm{soc}}$ and $\mathrm{TTC}_{\mathrm{econ}}$, commercial and noncommercial users would prefer a maintenance policy that minimizes the potential loss of capacity and that maximizes the consistency of provided service. In this example, Policy 2 (equal annual distributions) dominates all others in those two measures, as is indicated in Figure 26 and Figure 27. Policy 1, while preferred from a traditional engineering perspective, does not result in 
consistent levels of service over the entire planning horizon, and results in largest expected losses of traffic capacity over the entire planning horizon. ${ }^{3}$

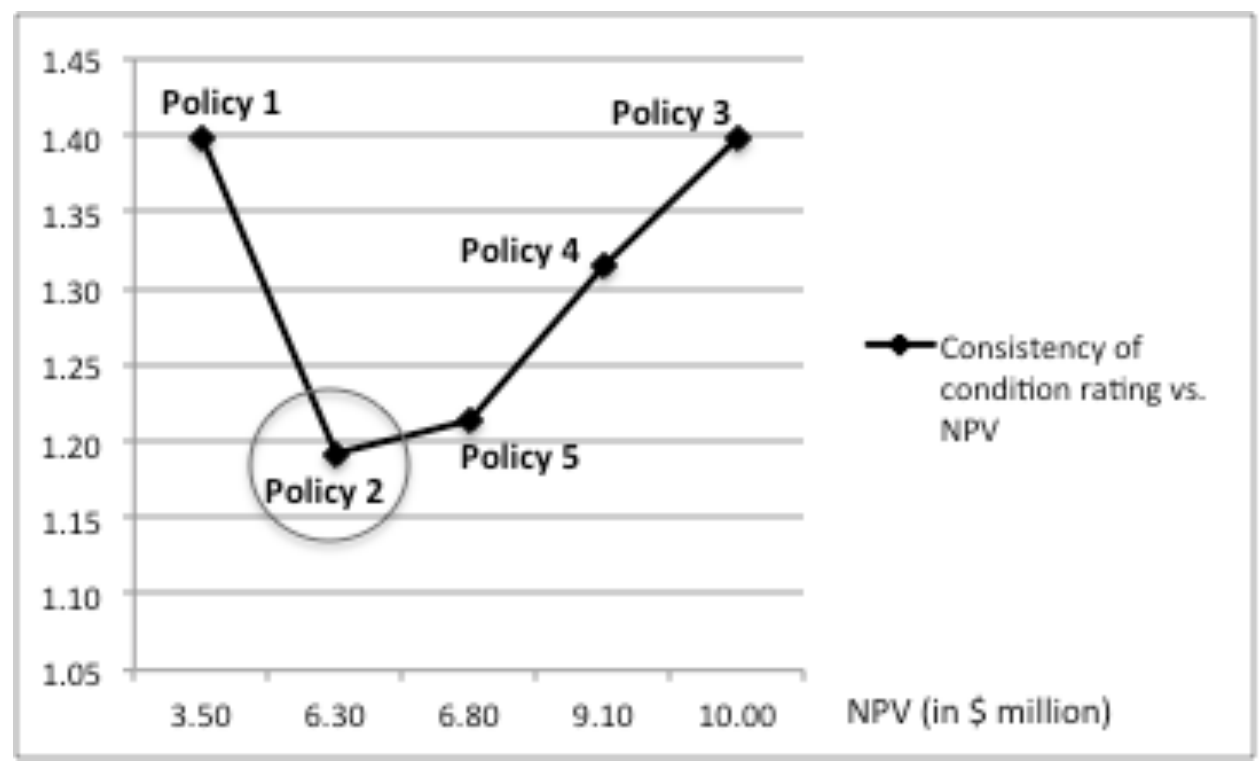

Figure 26: Consistency of superstructure condition rating versus NPV for the five maintenance policies. Consistency is calculated as the ratio between the maximum and minimum superstructure condition rating achievable under a certain policy over the entire planning horizon. We prefer values closer to 1.

When considering results from Figure 25, Figure 26, and Figure 27 we see that there is no maintenance policy that seems optimal from all three modeling perspectives. However, we suggest that the intrinsic meta-model can be used to guide the coordination of decentralized yet interdependent decisions made by the different stakeholders. Because of the coupling functions linking the shared state variable (bridge traffic capacity) to other essential variables of each submodel, stakeholders from the three sub-systems can more easily identify those factors (i.e., decisions, constraints) that might be adjustable either at the individual sub-system level, or through a collaborative effort, in order to reach a range of values of the bridge traffic capacity

\footnotetext{
${ }^{3}$ For this illustrative example, we assume that the average annual daily number of vehicles on the bridge at $k=0$ is 90,000 of which $\mathrm{x}_{\mathrm{soc}, 1}(0)=87,300(97 \%)$ are cars and personal vehicles (non-commercial) and $\mathrm{x}_{\mathrm{econ}, 1}(0)=2,700$ $(3 \%)$ are commercial trucks. The regional freight study conducted by the Hampton Roads Regional Planning District Commission (2007) suggests that these values are realistic in the urban area of interest.
} 
that would be acceptable to all stakeholders over the entire planning horizon, while still satisficing sub-system objectives.

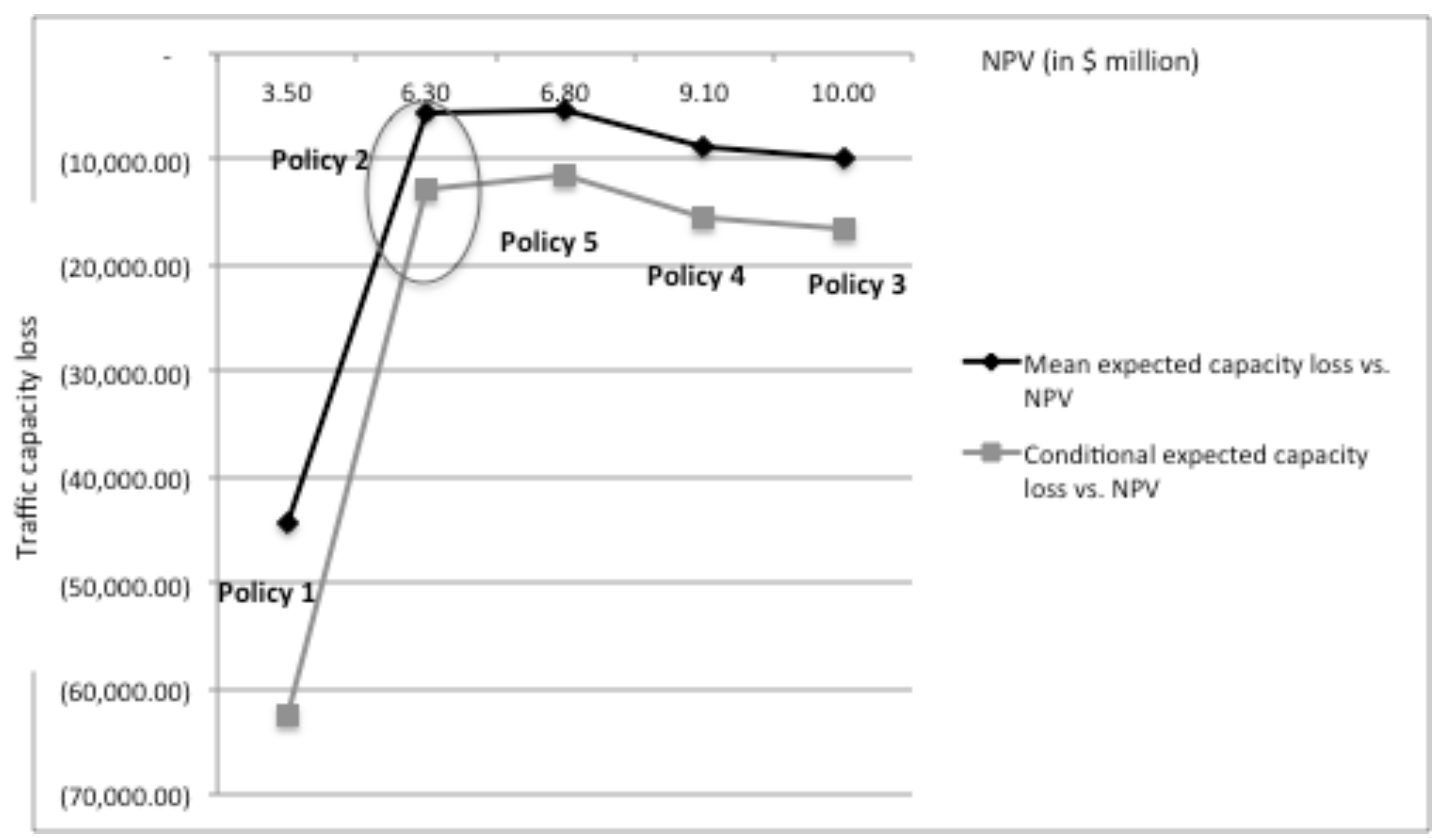

Figure 27: Aggregate values of the loss of bridge traffic capacity at period $k=T$. Bridge traffic capacity is compared at each $k$ to bridge demand, and additional capacity is recorded. This graph shows the sum of the additional bridge traffic capacity. We see that all five maintenance policies result in a lack of traffic capacity over the entire planning horizon.

DOT and MPO decision makers understand that their most preferred policy is not acceptable from a social and economic perspective. They understand that a more proactive maintenance plan, as proposed by Policy 2, is expected to produce more consistent superstructure condition and load ratings, thus ensuring a more consistent and adequate level of service over the entire planning horizon. However, when considered from a cost perspective, the NPV of Policy 2 is nearly double that of Policy 1 , hence Policy 2 will not be sustainable if additional innovative funding sources are not supplied.

In order to reduce the traffic demand on a deteriorated bridge, and hence address the issue of insufficient capacity caused by their preferred Policy 1, the DOT and MPO decision makers could put into place speed or weight postings. Speed or weight postings would increase the travel costs for commercial and non-commercial travelers, thus making Policy 1 even less attractive to stakeholders from the social and economic perspective. In order to make Policy 1 more 
acceptable from the social and economic perspectives, representative stakeholders could consider options that would reduce the number of commercial and non-commercial bridge users, including alternative transportation modes (e.g., buses), telecommuting work options, and others.

In order to make Policy 2 more acceptable from the engineering perspective, all stakeholders must consider how to implement innovative funding mechanisms to raise the required additional investments. Both commercial and non-commercial users could consider participating in shared infrastructure maintenance costs $\left(\mathrm{SIMC}_{\mathrm{soc}}\right.$ and $\mathrm{SIMC}_{\mathrm{econ}}$ in Eqs. $5-7 \mathrm{~b}$ and $\left.5-9 \mathrm{~b}\right)$ by paying increased fuel taxes, usage tolls, fees for transporting excessive weights over the bridge. While the participation in the shared infrastructure maintenance costs would increase the total cost per traveler, stakeholders could assess tradeoffs between increased SIMC and decreased TTC. For example, while the current level of maintenance investments made in Policy 2 is insufficient to maintain an excess bridge traffic capacity, an increase of $\$ 367 \mathrm{~K}$ at each period $k$, ensures the existence of the excess traffic capacity at each period $k$. If we very naively assume that the $\$ 367 \mathrm{~K}$ could be equally divided among all commercial and non-commercial bridge users at each stage $k$, then we see that a fairly small investment on behalf of infrastructure users (approximately $7.4 \%$ of $\mathrm{TTC}_{\mathrm{soc}}$ or $4.6 \%$ of $\mathrm{TTC}_{\text {econ }}$ every 2 -years) completely solves the bridge traffic capacity problem, while resulting in a sustainable and proactive bridge maintenance plan. On the other hand, assume that Policy 1 is implemented, resulting in a speed and a weight posting. Based on the assumptions of our meta-model, such a policy could increase the $\mathrm{TTC}_{\mathrm{soc}}$ and $\mathrm{TTC}_{\text {econ }}$ by approximately $3 \%$ for every one thousand miles traveled. Stakeholders representing the social and economic perspectives could then evaluate tradeoffs between these alternatives to determine which option results in more acceptable long-term system of systems solutions.

The goal of the intrinsic meta-modeling as applied to the bridge maintenance problem is to provide a tool through which stakeholders could envision how their own actions impact the interdependent sub-systems, and how in the long run, actions that may seem optimal from a single perspective, may in fact be harmful to the overall system of systems. Thus, through the process of meta-model coordination, we enable stakeholders to identify decisions or constraints that should be manipulated (either in a single sub-system, or through a collaborative effort of multiple sub-systems) so as to achieve values of shared state variables that are more acceptable 
from all modeling perspectives. Every time a new effort is made to adjust a local decision/constraint, new Pareto-optimal solutions must be computed for each sub-system based on suggested changes, and based on those local optimization results, new projected ranges of shared state variables must be re-evaluated. This process continues until a solution is found that satisfices local sub-system objectives while also resulting in levels of shared state variables that are acceptable to all stakeholders over a longer planning horizon. A failure of sub-systems to agree on a globally-acceptable range of values of shared state variables could manifest in local sub-system difficulties which could, over time, propagate and become detrimental to the entire system of systems.

\subsection{Brief summary}

While the sub-models and assumptions presented in this case study are simplistic, they provide a first step in understanding the potential value of meta-modeling through shared state variables. The implemented modeling process encourages different stakeholders to identify, quantify and explicitly model some common and conflicting interests and needs, and it provides guidance about the extent to which the knowledge of shared variables can help in making decisions in a decentralized environment characterized with multiple and conflicting stakeholders and objectives.

In particular, the intrinsic meta-modeling process enables DOT and MPO decision makers to get a macro-level estimate of the potential socio-economic impacts of their maintenance alternatives. Hence, their ultimate maintenance choices are informed and guided by considerations of how the maintenance decisions made today impact the choice of future maintenance options, and how they affect other system stakeholders. Based on this knowledge, other system stakeholders, including commercial and non-commercial users of the infrastructure, can evaluate the impacts that infrastructure maintenance decisions have on them, given their changing needs, demands and constraints. Maintenance plans resulting in severe consequences to stakeholders can be collaboratively re-evaluated by considering, among others, whether: (i) other, less damaging alternatives exist, (ii) innovative funding mechanisms (partially supported by infrastructure users) could be designed to support alternative projects, and (iii) consequences to 
infrastructure users could be temporarily assuaged (e.g., by utilizing more alternate routes or different public modes of transportation). This type of a collaborative, forward-looking management approach maintains and potentially increases, within a limited budget, the number of available maintenance options in the future thus transforming an operational, short-term approach into a strategic, long-term and collaborative approach. Klosterman (1999) emphasizes the need for collaborative planning approaches for public infrastructures by suggesting that "planning and policy making should not be based on closed and unsupervised "objective" analysis of technical experts," and by stating that "planners are increasingly abandoning the attempts to "plan for" the public for more collaborative efforts to "plan with" the public." 


\section{Chapter 6: Broader Public Policy Considerations ${ }^{4}$}

This dissertation addressed two major concerns - that of creating generic approaches for modeling and management of systems of systems, and that of applying such approaches to support more sustainable and forward looking management of the deteriorated U.S. bridge infrastructure. In particular, this dissertation presented a system of systems modeling framework that builds on the theory of PSM intrinsic meta-modeling, and allows for modeling of systems of systems through multiple models that are harmonized at the meta-level via shared state variables. The case study presented in Chapter 5 illustrated how intrinsic meta-modeling via shared state variables can aid stakeholders involved with different sub-systems to better understand the impact that their decisions have on the other sub-systems, and it pointed to the need to adopt a more forward-looking and collaborative public policy for infrastructure maintenance. However, adopting a new modeling and management paradigm is not in itself easy or sufficient, and additional incentives and commitment devices will be needed in order to start making improvements in how the transportation infrastructure is managed.

In reality, most infrastructure maintenance decisions are reactive and little value is placed on proactive management that considers long-term aspects and diverse stakeholder needs. There are many reasons for this including, among others, (i) the U.S. political framework and culture, (ii) a societal culture that values individualism over collaboration, (iii) the psychological perceptions of risk, and (iv) the lack of public accountability. These closely intertwined factors frame the extent of possible solutions in infrastructure management.

There are many characteristics of the American political and policymaking process and framework that tend to discourage long-term investments in infrastructure. The relatively short electoral cycle for most elected officials (e.g., three or four year terms of office for elected mayors and city councilors, and a mere two year cycle for U.S. House of Representatives) encourages emphasis on tangible short-term outcomes and results. Longer-term investments (e.g.,

\footnotetext{
${ }^{4}$ Discussion in this chapter is based on Andrijcic et al. (2013)
} 
ten or more years into the future) are difficult for a politician to justify or campaign around. Investments in infrastructure, moreover, yield benefits that are often very difficult for constituents to visualize or fully appreciate. In a way similar to strong building codes that prevent structures from falling during an earthquake, the failure of a negative event to occur garners little media attention, and is hard for an elected official to claim credit for. Similarly, the fact that a bridge does not fail (and remains open for traffic), is not notable or newsworthy, and is not something for which constituents are thankful for and apt to reward their elected representatives for. Thus, this creates a general attitude that infrastructure “maintenance isn't sexy" (ASCE, 2009b). Politicians have a direct gain from building new infrastructure, yet they lose nothing if an infrastructure fails, because that loss is transferred to somebody else.

When the solutions are costly, complex, and controversial, or involve a wide range of players, our tendency, as individual communities and as a nation, is to stall and point our fingers at the "other guy" until we reach a crisis (Caldwell, 2011).

The positive benefits associated with regular long term investments in upkeep and maintenance thus are mostly "unseen" by the public, making them harder to justify politically. This is partly to do with the psychological perception of risk in which individuals respond more strongly to an outcome that results from action than to the same outcome that results from inaction (Kahneman, 2011). Thus, the general public is less critical of a bridge failure when it occurs as a result of untimely and inadequate maintenance, than when it occurs after a particular maintenance action has taken place.

Furthermore, the general public often does not understand the value of proactively investing in infrastructure because they do not understand the complexity of the infrastructure and how the changes in the performance of the infrastructure affect them directly (ASCE, 2009b). Recent reports (ASCE, 2009b) suggest that one of the challenges of improving infrastructure management will be to investigate ways in which decision makers can educate the public about the need of proactive maintenance. At the moment, the political system is often "guided by the intensity of public sentiment" (Kahneman, 2011) therefore an infrastructure failure usually precedes a management action. 
There are other features of the American policy and political scene that similarly discourage long-term investments. The famously fractured and fragmented nature of American governance structure is another impediment. While strong regional planning is common in Europe (and in others places, such as Australia), for instance, this level of governance is almost non-existent in the U.S.. Rather, the U.S. is characterized by thousands of local government units, each often competing with each other for projects and tax base, and with little incentive to adopt and implement a larger infrastructure plan or vision. Most infrastructure projects - whether bridges or highways or subway systems -require regional plans, and regional mechanisms of financing and operation.

The prevailing political culture also plays an important role here. The unique political history of the U.S. emphasizes small government, state's rights and general suspicion of federal largess and leadership. Politicians and elected officials today are held to a standard that a priori questions the value of government expenditures (in contrast to decisions and investments made in the private sector). Raising taxes, moreover, is philosophically difficult in the conservative political climate, and often perceived to be politically suicidal by many elected officials. In contrast to Europe, where the role of strong central governments is accepted, and investments in public infrastructure are seen as an important and essential government function, the American political culture and climate are more skeptical. Equally true, there are many competing interests and policy areas, vying for limited public funds. Evidence suggests, for instance, that longerterm policy issues and topics (natural hazard mitigation, climate change) are likely eclipsed, especially at the local level, by a host of other more immediate demands on budgets - fire and police service, for instance, as well as schools.

Given these challenges, a major shift in accountability will be required on behalf of all decision makers and all users in order to improve the current state of the transportation infrastructure. Engineering solutions on their own will not suffice, and changes will have to be made on the regulatory and user side as well (ASCE, 2009a). In order to provide more sustainable funding mechanisms, all users will have to participate in the sharing of infrastructure maintenance costs (Caldwell, 2011). While this idea might be objectionable to infrastructure 
users $^{5}$, it is important to note that by relegating maintenance as a last minute effort, most risks are experienced by the users, both commercial and non-commercial (e.g., higher vehicle maintenance costs, increased travel time and cost, lost opportunity cost). Furthermore, a refusal to invest in infrastructure maintenance proactively will increase the funding gap and the number of deficient infrastructure assets, and this will increase the cost of future infrastructure improvements exponentially:

When infrastructure maintenance, repairs, and improvements are not fully funded, short-term "band-aid" solutions are often implemented to enable the infrastructure to continue functioning at less than minimum tolerable conditions. When these short-term solutions are implemented, in addition to the user cost of operating the deficient infrastructure, the cost of operating and maintaining the infrastructure is greater than it would be if the infrastructure were in proper condition. (ASCE, 2011)

More proactive maintenance, while seemingly unfeasible at times of fiscal constraint, provides a mechanism for better risk sharing among all stakeholders, and reduces future costs. In order to ensure a more proactive approach to infrastructure management, changes need to be made at all levels of the decision making process. This can be accomplished more efficiently and successfully if transportation infrastructure management is addressed as a system of systems problem that can be successfully solved only by considering a wide range of stakeholder perspectives, inclusive of engineering, social, economic, environmental, political and normative dimensions, as was discussed in preceding chapters. But, simply changing the way in which we model and manage the transportation infrastructure is not sufficient. At the end of the day, the question that remains is, where does the additional money come from?

One way in which some foreign countries have ensured a more proactive maintenance of transportation infrastructure assets has been through the so-called public-private partnerships

\footnotetext{
${ }^{5}$ A poll recently conducted by the Rockefeller foundation found that over 75 percent of voters believe that improving the country's transportation infrastructure is "highly important," but almost all voters are opposed to helping to pay for the needed improvements (Strauss, 2012). In particular, "71 percent oppose increasing the gas tax, 64 percent oppose new tolls on existing roads and bridges, and 58 percent oppose paying more for each mile driven" (Strauss, 2012). However, some local governments have been able to raise additional funding to support local infrastructure projects by increasing sales taxes (Strauss, 2012).
} 
(PPPs) in which private sector organizations participate in the delivery and/or financing of public infrastructure projects (Istrate and Puentes, 2011). The structure of the PPPs differs greatly from project to project, and includes everything from complicated concession agreements and availability payments (Puentes, 2009) to truck tolls that impose higher fees on heavy trucks that damage the surface transportation assets more (Building America's Future Educational Fund, 2011). Ideally PPPs should not only provide the additional financing, but they should also serve as an instrument for risk and cost sharing (Istrate and Puentes, 2011). Forrer et al. (2010) suggest that "PPPs display a variety of these horizontal relationships through collaborative mixing, consensual decision making, and other recognized characteristics of organizational partnerships."

While many foreign countries, including Canada (Puentes, 2009), are employing PPPs at a high level to finance their transportation infrastructure projects, the U.S. is lagging behind on all measures (Istrate and Puentes, 2011). "The U.S. is now one of the only leading nations without either a national plan for public-private partnerships (PPPs or P3s) for infrastructure projects or a national infrastructure bank to finance large-scale projects and harness private capital" (Building America's Future Educational Fund, 2011). Of the 29 U.S. states implementing PPPs, Florida, California and Texas have the highest number of implemented PPPs, while Virginia, California and Michigan have dedicated PPP units that aim to systematically deliver innovative financing mechanisms for transportation projects (Istrate and Puentes, 2011; Strauss, 2012). However, there are very few large-scale PPP projects in the U.S.. Notable exceptions include the Indiana Toll Road and Chicago Skyway projects (Strauss, 2012). Experts agree, however, that existing infrastructure funding practices are insufficient, and that with further budget cuts and a more "favorable political climate" (Strauss, 2012) more PPPs are likely to occur in the future:

... the traditional means of raising transportation dollars is almost out of gas and cannot keep pace with demographic and market forces, metropolitan development patterns, the aging of the nation's existing infrastructure, and demands for upgrading the outmoded infrastructure we currently have. (Puentes, 2009)

At the September 25, 2010 American Road and Transportation Builders Association conference, aides from both political parties "acknowledged that, by necessity, such public-private deals will play a part in future funding, 
especially in light of the congressional reluctance to increase the gas tax that is the main source of federal transportation revenue. (Dannin, 2011)

Currently approximately 25 percent of all transportation investments come from the federal government, and the rest come from the state and local governments (Building America's Future Educational Fund, 2011). Federal funds, known as the Highway Trust Fund, are primarily collected from the federal gas taxes that are not indexed to inflation and that have not increased since the early 1990s. These funds have further dwindled due to the sudden increase of fuelefficient cars (Building America's Future Educational Fund, 2011). The Highway Trust Fund has been unable to "meet its spending obligations since 2008," and the Congress has had to appropriate general funds to settle the shortage (Strauss, 2012). The Congressional Budget Office estimates that given the current expenditure levels by 2021 "the highway fund will face annual deficits of about $\$ 17$ billion and a cumulative deficit of $\$ 169$ billion" (Strauss, 2012). Thus, the need for additional transportation infrastructure investment is obvious, yet the "the U.S. has not fostered an environment in which the private sector will step in to help finance the large-scale infrastructure projects we need" (Building America's Future Educational Fund, 2011). The underlying environment is characterized by a lack of trust between the government and the private sector. In order for transportation PPPs to be successful in the U.S.:

... the private sector needs to approach transportation projects with a strong sense of commitment and understanding of the public interest. This will not happen without the federal government providing (a) clarity of objectives, (b) clear understanding of roles and responsibilities, and (c) consistency of decision-making. Currently the U.S. is failing on all three points. (Puentes, 2009)

There are significant "procedural issues that must be resolved if we are to have the high quality infrastructure necessary to meet this nation's needs and further its goals and if we are to achieve those goals by an open and democratic process" (Dannin, 2011). One of the issues that must be addressed is the concern of public good and public support. This cannot be achieved without a more transparent management approach that informs the public and all stakeholders of the costs, benefits and trade-offs related to infrastructure maintenance decisions. Currently there is a strong sense of public opposition to the increased tolls and fees. This opposition stems 
primarily from the inability of the public to understand the benefits of more proactive infrastructure investments (Dannin, 2011).

While the general public has thus far primarily resisted increased taxes for supporting proactive maintenance of infrastructures, the intrinsic meta-modeling approach utilized in this dissertation provides a more transparent process through which all stakeholders might better understand how changes in the performance of infrastructures affect them directly, thus enabling them to make more informed trade-offs. The intrinsic meta-modeling approach also enables decision makers and stakeholders to better understand that by relegating infrastructure maintenance as a last minute effort most risks and costs are experienced by the users, and that inaction on part of any single stakeholder constitutes disaster in the long run. Additionally, the approach enables commercial and non-commercial infrastructure users to compare the costs that they might experience from existing maintenance policies to the costs of participating in infrastructure maintenance through tolls or other programs.

By bringing the engineering evidence to the political arena, the discussed methodology provides a justification for action, and illustrates the need to account for the varying and often conflicting stakeholder perspectives. Results presented in Chapter 5 point to the need to adopt a more forward-looking and collaborative public policy for bridge infrastructure maintenance. In conclusion, this dissertation provides a theoretical framework and a methodological approach for creating models in which interdependencies between different stakeholder and system perspectives are more transparent and better understood by all stakeholders. As suggested by Paul Slovic, in our quest to improve risk management practices "it is far more depressing ... to fail to understand the complex psychological, social, cultural and political forces that dictate the successes and failures of risk management" (Slovic, 2000).

While new analytical approaches are needed to support a long-term infrastructure investment scheme, ultimately there are other issues that will need to be addressed to effect the change. One solution might be to begin celebrating and acknowledging the making of long-term maintenance investments, by converting these investment decisions into visible public events analogous to the conventional ribbon cutting ceremonies for newly built projects or facilities. This has essentially been done in the anti-terrorism efforts, where decisions made to avert terrorist events are celebrated and financed. Another solution may be to form political constituencies, similar to the 
American Association of Retired Persons (AARP) or the National Education Association (NEA), which would lobby politically on behalf of infrastructure decisions and investments to make them more visible and attractive. Yet another solution may be to find new ways of educating the general public about the importance of long-term infrastructure investments. Perhaps civics and citizenship classes at the elementary and high school level could include discussions on public infrastructure, thus instilling an understanding and a sense of the civic duty of providing basic and essential infrastructure investments. Another long-term solution might be to create structures and mechanisms that would ensure that adequate funds are available for maintenance - perhaps these could be analogous to the Social Security at the individual level. Or, they could be structured as variations of the existing federal transportation loan program called Transportation Infrastructure Finance and Innovation Act (TIFIA), which was introduced in 1998 to provide federal credit assistance to "leverage private capital and finance large-scale surface transportation projects undertaken by public or private entities" (Strauss, 2012).

Finally, there exists a need to re-visit and reinvigorate a strong political and ethical underpinning for what the "public interest" requires. We should perhaps revisit basic notions of the "compact" or "social contract" that a free and democratic society assumes and requires. Investing in the basics of infrastructure is an essential part of this broader discussion about what we can and should reasonably expect society to provide. It is time to revisit these basic underlying political philosophical questions and begin to have a national debate about what the social contract entails. Such a national dialogue, coupled with a more system of systems oriented analytical approach, should result in a new found ethical and political underpinning for long term investments in infrastructure. Such investments are a matter of fairness and a legitimate expectation of American citizens. 


\section{Chapter 7: Conclusion}

This dissertation presented methodological and theoretical extensions to the PSM intrinsic metamodeling theory for systems of systems (Haimes, 2012). In particular, this dissertation introduced an iterative, learn-as-you-go methodological process for the construction of interdisciplinary models that represent different perspectives of a system of systems, and for their integration into a single meta-model through the use of shared state variables. From a theoretical perspective, this dissertation presented two contributions. First, we demonstrated through a simplistic physical system of systems that the knowledge of shared state variables transforms a black box system identification problem into a more informative grey-box system identification problem. Second, this dissertation provided a theoretical discussion of how the meta-model can be used as an instrument for the meta-level coordination of decentralized, sub-systems' decisions. Finally, this dissertation demonstrated through a case study how the developed theory and methodology could be applied to the U.S. bridge infrastructure system of systems to examine the broader effects of untimely or insufficient bridge maintenance, with the purpose of creating more holistic and adaptive public policy recommendations for civil infrastructures.

The developed intrinsic meta-modeling process motivates different stakeholders to identify, quantify, and explicitly model some common and conflicting interests and needs, with the goal of creating policies that are satisficing to all sub-systems, and cognizant of, and responsive to, emergent risks as identified from different stakeholder perspectives. Given the evolving nature of objectives, interest groups, organizational, political, and budgetary baselines, and the requirements associated with interdependent sub-systems, the meta-modeling coordination through shared state variables enables all stakeholders to project and prepare for future changes and to achieve outcomes that are superior to uncoordinated policies developed without the metamodeling coordination.

While the case study presented in this dissertation is fairly simplistic and macro-level, it provides a first step in understanding the potential value of meta-modeling through shared state variables. Thus, this dissertation provides the much needed guidance regarding the importance of shared variables/objectives/interests in making decisions in a decentralized environment 
characterized by multiple and conflicting stakeholders and objectives. In particular, the intrinsic meta-modeling process enables DOT and MPO decision makers to obtain a macro-level view of the potential socio-economic impacts of their maintenance alternatives (estimates which can then be assessed in more detail through more specific models that have been identified in literature (see Chapter 2)). Hence, the decision makers' ultimate maintenance choices are informed and guided by considerations of how the maintenance decisions made today impact the choice of future maintenance options, and how they affect other system stakeholders. Based on this knowledge, other system stakeholders, including commercial and non-commercial users of the infrastructure, can evaluate the impacts that infrastructure maintenance decisions have on them, given their changing needs, demands and constraints. Maintenance plans resulting in severe consequences to stakeholders can be collaboratively re-evaluated by considering, among others, whether: (i) other, less damaging alternatives exist, (ii) innovative funding mechanisms (partially supported by infrastructure users) could be designed to support alternative projects, and (iii) consequences to infrastructure users could be temporarily assuaged (e.g., by utilizing more alternate routes or different public modes of transportation). This type of a collaborative, forward-looking management approach maintains and potentially increases, within a limited budget, the number of available maintenance options in the future thus transforming an operational, short-term approach into a strategic, long-term and collaborative vision. By bringing the engineering evidence to the political arena, the discussed methodology provides a justification for action, and illustrates the need to account for the varying and often conflicting stakeholder perspectives. Moreover, the insights obtained from the system of systems methodological approach point to the need to adopt a more forward-looking and collaborative public policy for infrastructure maintenance.

Possible future extensions of the developed work include the following. The case study presented in this dissertation assumed that the engineering, social and economic macro-level changes occurring due to insufficient or untimely bridge maintenance can be adequately represented through linear and time invariant models. The purpose of this dissertation was to demonstrate the potential value of modeling a large civil infrastructure from multiple perspectives - engineering, societal, and economic - and coordinating and integrating the three modeling perspectives via the proposed meta-modeling approach. Therefore, the sub-models developed and deployed in Chapter 5 were fairly simplistic and adopted an LTI structure. The 
LTI structure was utilized because it provides closed form solutions, implies constant parameters, and enables us to compare trade-offs between objective functions of the different sub-systems. Moreover, the state space representation reflects our concern with the time domain that is critically important in the risk management process. While our assumption that the structural, social and economic changes to the bridge system of systems follow a linear pattern was convenient for several aforementioned reasons, it does not reflect the reality well under all conditions. Therefore, work could be done to extend this work by demonstrating the intrinsic meta-modeling coordination process for non-linear models. One of the proposed future extensions could include the development of non-linear sub-models for all three perspectives to better account for the impact of time delays and travel-cost reactions, progressive increases in vehicle weights and sizes, substitutions of work and shopping with on-line shopping on travel patterns and hence on the structural condition of the bridge. This could be done by augmenting analytical approaches with agent-based modeling and simulation.

Another possible extension of this work includes creating a computerized decision support tool that would operationalize the theory and methodology developed in this dissertation. While the benefits of collaborative modeling and management efforts for critical infrastructure systems are obvious, they are not easy to implement in practice. This is especially true in a decentralized and distributed environment where there might exist many obstacles that would prevent stakeholders from working together in real time. Hence, creation of a simulated collaborative environment in which stakeholders can visualize all of the available knowledge from different perspectives, and can work individually at their own leisure while being able to visualize the impact that their decisions might have on the entire system of systems, would make this approach more appropriate and applicable to real world conditions. An additional possible extension includes the creation of more streamlined and detailed engineering, economic and social sub-models that would be more scalable and applicable to a larger number of bridges. 
(This page intentionally left blank) 


\section{Appendix I: Derivation of a First Principles Model For the Two-Component Thermodynamic System of Systems ${ }^{6}$}

In the absence of an adequate testing facility in which we could measure actual observations for the two-component thermodynamic system, we developed a physical model based on first principles, which we then utilized to generate input and output observations. We used those generated observations to train and validate the statistical models identified in Chapter 3. The derivation of the physical model based on first principles is described below.

We assume that (i) the rate of heat transfer between two rooms is linearly proportional to the temperature difference between two rooms, (ii) the air temperature is evenly distributed in the room all the time, (iii) there is no heat exchange between the rooms and the environment, (iv) the mass of air in each room is kept constant, and (v) the material heat coefficient for air is constant with temperature. The following notations are used:

$t: \quad$ Time

$W_{i}(t): \quad$ Accumulated work to air conditioner i observed at time $\mathrm{t}$

$P_{i}(t): \quad \quad \quad$ Power to air conditioner i observed at time $\mathrm{t}$

$T_{L i}(t)$ : Temperature of room i observed at time $\mathrm{t}$

$T_{S i}$ : $\quad$ Starting inside temperature of room i (constant)

$T_{T i}(t)$ : Target inside temperature of room $\mathrm{i}$ at time $\mathrm{t}$

$T_{H}: \quad$ Environment temperature (constant)

$Q_{L i}: \quad$ Heat removed from room i by air conditioner

$Q_{H i}$ : $\quad$ Heat output to environment from air conditioner i

\footnotetext{
${ }^{6}$ This appendix is based on a working paper by Haimes et al. (2013); results were obtained in collaboration with Ph.D. candidate Zhenyu Guo.
} 
$Q_{\text {Trans }}: \quad$ Heat transferred between two rooms due to temperature difference

$Q_{i}$ : $\quad$ Heat from the change of air temperature in room i

$m_{i}: \quad$ Mass of air in room i (constant)

$c_{p}$ : $\quad$ Material heat coefficient for air (constant)

$\alpha: \quad$ Coefficient of heat transfer between two rooms (constant)

$\beta$ : $\quad$ Efficiency of air conditioner performance (constant)

For an air conditioner the following properties hold true according to the $1^{\text {st }}$ law of thermodynamics (Moran \& Shapiro, 1996):

$$
Q_{H}=Q_{L}+W
$$

From the $2^{\text {nd }}$ law of thermodynamics, we have:

$$
\frac{Q_{L}}{W}=\beta \cdot\left(\frac{1}{\frac{T_{H}}{T_{L}}-1}\right)
$$

where for $\beta=1$, this is the efficiency of a Carnot cycle or ideal engine. $\mathrm{T}$ is absolute or $\mathrm{K}$ scale. The relation between work and power can be expressed as:

$$
P=\dot{W}=\frac{d W}{d t}
$$

Based on Eqs. A1-2 and A1-3, we have:

$$
\frac{\dot{Q}_{L}}{\dot{W}}=\frac{\dot{Q}_{L}}{P}=\beta \frac{1}{\frac{T_{H}}{T_{L}}-1}
$$


at a steady-state; i.e. $T_{H}$ and $T_{L}=$ constant.

The relation between heat and the change of air temperature is expressed as:

$$
Q=-c_{p} m \Delta T_{L}
$$

and

$$
\dot{Q}=-\frac{d Q}{d t}=-c_{p} m \frac{d T_{L}}{d t}
$$

The relationship describing temperatures and heat transfer between the two rooms is:

$$
\dot{Q}_{\text {Trans }}=\alpha\left(T_{L 1}-T_{L 2}\right)
$$

Based on these principles we derive the system dynamic equations for the two rooms under consideration. For Room 1,

$$
Q_{1}=Q_{L 1}+Q_{\text {Trans }} \text { and } \dot{Q}_{1}=\dot{Q}_{L 1}+\dot{Q}_{\text {Trans }}
$$

When we substitute Eqs. A1-4, A1-6, and A1-7 into A1-8 we get:

$$
-c_{p} m_{1} \frac{d T_{L 1}(t)}{d t}=P_{1}(t) \beta \frac{1}{\frac{T_{H}}{T_{L 1}(t)}-1}+\alpha\left(T_{L 1}(t)-T_{L 2}(t)\right)
$$

From Eq. A1-9, we obtain the expression for power $P_{1}(t)$ as a function of temperatures $T_{L 1}(t)$ and $\mathrm{T}_{\mathrm{L} 2}(\mathrm{t})$.

$$
P_{1}(t)=\frac{\frac{T_{H}}{T_{L 1}(t)}-1}{\beta}\left[-c_{p} m_{1} \frac{d T_{L 1}(t)}{d t}-\alpha\left(T_{L 1}(t)-T_{L 2}(t)\right)\right]
$$


Then work of air conditioning in Room 1 can be expressed as:

$$
W_{1}(t)=\int_{0}^{t} P_{1}(t) d t
$$

Similarly, for Room 2, we have:

$$
Q_{2}=Q_{L 2}-Q_{\text {Trans }} \text { and } \dot{Q}_{2}=\dot{Q}_{L 2}-\dot{Q}_{\text {Trans }}
$$

When we substitute Eqs. A1-4, A1-6, and A1-7 into A1-12 we get:

$$
-c_{p} m_{2} \frac{d T_{L 2}(t)}{d t}=P_{2}(t) \beta \frac{1}{\frac{T_{H}}{T_{L 2}(t)}-1}-\alpha\left(T_{L 1}(t)-T_{L 2}(t)\right)
$$

From Eq. A1-13, we obtain the expression for power $P_{2}(t)$ as a function of temperatures $T_{L 1}(t)$ and $\mathrm{T}_{\mathrm{L} 2}(\mathrm{t})$.

$$
P_{2}(t)=\frac{\frac{T_{H}}{T_{L 2}(t)}-1}{\beta}\left[-c_{p} m_{2} \frac{d T_{L 2}(t)}{d t}+\alpha\left(T_{L 1}(t)-T_{L 2}(t)\right)\right]
$$

Then work of air conditioning in Room 2 can be expressed as:

$$
W_{2}(t)=\int_{0}^{t} P_{2}(t) d t
$$

In order to develop an empirical model of the system, we assume that the outside temperature is $T_{M}=35^{\circ} \mathrm{C} \approx 308 \mathrm{~K}$, the initial inside temperatures of the two rooms $T_{S 1}=32^{\prime \prime} \mathrm{C} \approx 305 \mathrm{~K}$ and $T_{S 2}=30^{\circ} \mathrm{C} \approx 303 \mathrm{~K}$, and the target inside temperatures of both rooms is $T_{Y_{1}}=T_{\gamma_{2}}=25^{\circ} \mathrm{C} \approx 29 \mathrm{BK}$. Furthermore, we assume that Room 1 contains $600 \mathrm{~kg}$ of air and 
Room 2 contains $300 \mathrm{~kg}$ of air. To simplify the problem we assume that $c_{p}=\frac{1.012 \mathrm{~J}}{g K}, \alpha=500$ and $\beta=0.65$. The temperature of both rooms needs to be decreased linearly from the initial temperature to the target temperature in 1,000 seconds ( 16.7 minutes).

$$
T_{L 1}(t)=-0.007 t+305 \text { and } T_{L 2}(t)=-0.005 t+303
$$

Based on these specifications, Eqs. A1-10, A1-11 and A1-14, A1-15 are transformed into:

$$
\begin{aligned}
& P_{1}(t)=\frac{(t+3250.4)}{0.65}\left[\frac{308}{-0.007 t+305}-1\right] \\
& W_{1}(t)=-0.7692 t^{2}-72693 t-3.17 \times 10^{9} \log (-7 t+305000)+3.17 \times 10^{9} \log (305000) \\
& P_{2}(t)=\frac{(-t+2518)}{0.65}\left[\frac{308}{-0.005 t+303}-1\right] \\
& W_{2}(t)=0.7692 t^{2}+90895.4 t+5.5 \times 10^{9} \log (-t+60600)-5.5 \times 10^{9} \log (60600)
\end{aligned}
$$

Eqs. A1-18 and A1-20 are used to generate input observation data and Eq. A1-16 is used to generate the output observation data. The training dataset is derived from Eqs. A1-18, A1-20 and A1-16 for the period $t$ from 0 to 1000 seconds. The validation dataset is derived from Eqs. A1-18, A1-20 and A1-16 for the period $t$ from 1000 to 2000 seconds. Data points for Scenario 2 in Chapter 3 (i.e., contaminated partial dataset) are derived from Eqs. A1-18, A1-20 and A1-16 for the period $t$ from 0 to 1000 seconds, with the addition of random noise to all parameters. The R-code utilized for regression equations and model comparison is as follows: 


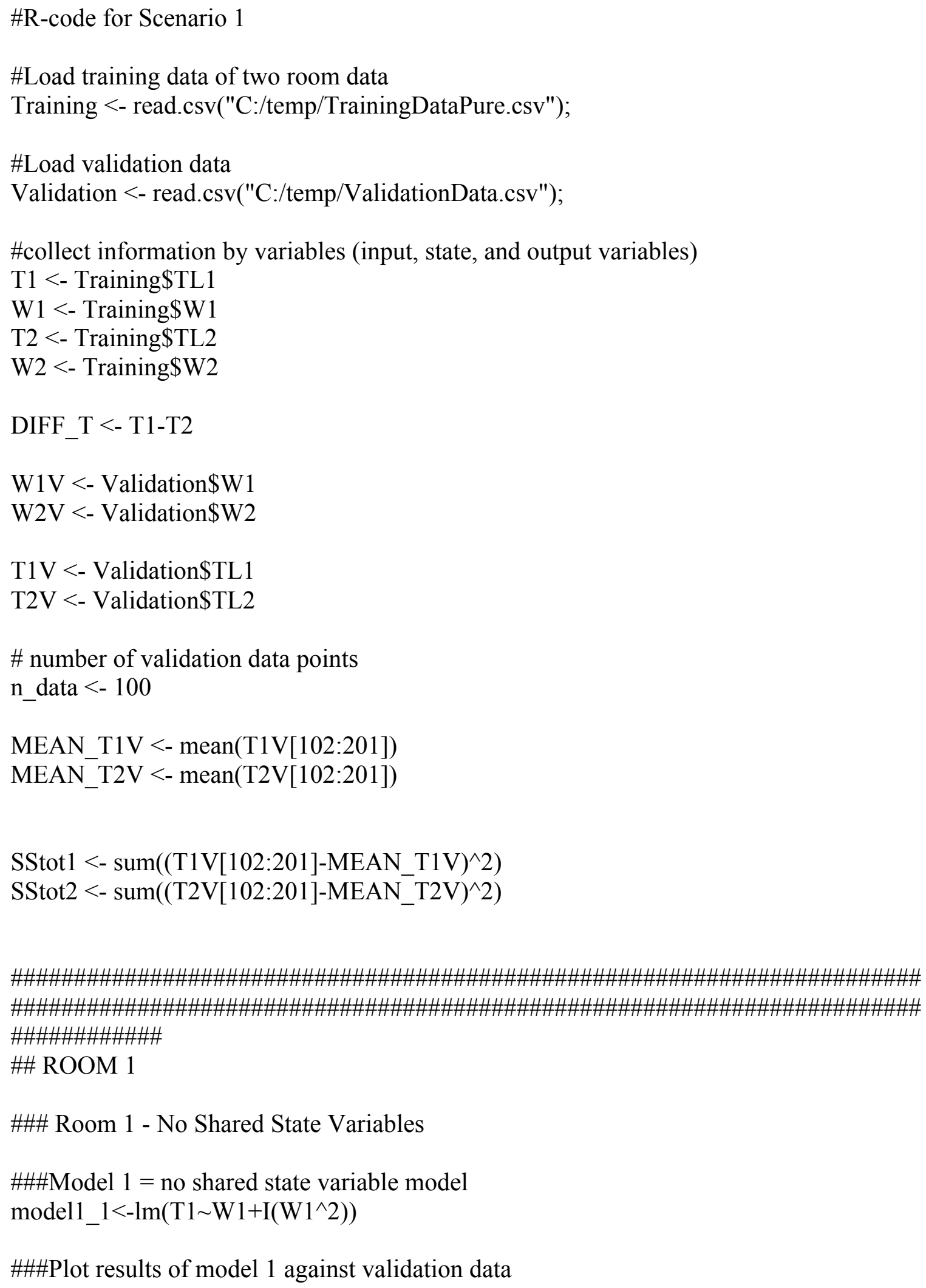


plot(Validation\$t,Validation\$TL1,type="l",col="red", ylim=c(265,340),xlab="Time (s)",ylab="Temperature (K)",main="Room 1 (Red: Validation Data; Blue = No Shared SV; Green: With Shared SV)")

lines(Validation\$t,model1_1\$coefficients[[1]]+model1_1\$coefficients[[2]]*W1V+model 1_1\$coefficients $[[3]]^{*} \mathrm{~W} 1 \overline{\mathrm{V}}^{\wedge} 2, \mathrm{col}="$ blue")

\#\#\#Performance of Model 1

SSerr $11<-$

$\operatorname{sum}\left((\text { model1_1\$coefficients[[1]]+model1_1\$coefficients[[2] }]^{*} \mathrm{~W} 1 \mathrm{~V}[1: 100]+\right.$ model1_1\$ coefficients $\left.\left.[[\overline{3}]]^{*} \mathrm{~W} 1 \mathrm{~V}[1: 100]^{\wedge} 2-\mathrm{T} 1 \mathrm{~V}[1: 100]\right)^{\wedge} 2\right)$

MSE11<-SSerr11/n_data

n_regressor $<-2$

R_sqr11<-1-SSerr11/SStot1

R_sqr_adj11<-1-(1-R_sqr11)*(n_data-1)/(n_data-1-n_regressor)

AIC $11<-\log (\operatorname{MSE} 11)+\left(\mathrm{n} \_\right.$data $+2 *$ n_regressor $) / n \_$data

$\mathrm{AICc} 11<-\log (\mathrm{MSE} 11)+\left(\mathrm{n} \_\right.$data $+\mathrm{n}$ _regressor $) /\left(\mathrm{n} \_\right.$data-n_regressor-2)

$\mathrm{BIC} 11<-\log (\mathrm{MSE} 11)+\mathrm{n} \_$regressor* $\log \left(\mathrm{n} \_\right.$data $) / \mathrm{n} \_$data

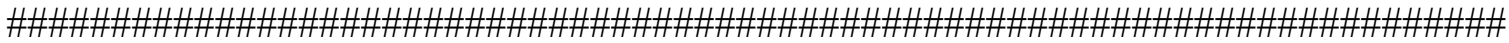
\#\#\#\#\#\#

\#\#\# Room 1 - Shared State Variable $=$ T1 - T2

\#\#\#Model 2 = shared state variable model

model1_2<-lm(T1 W1+I(W1^2)+DIFF_T $)$

\#\#\#Plot results of model 2 against validation data

lines(Validation\$t,model1_2\$coefficients[[1]]+model1_2\$coefficients[[2]]*W1V+model $1 \_2$ scoefficients $[[3]]^{*} \mathrm{~W} 1 \mathrm{~V}^{\wedge} 2+$ model1_2\$coefficients[[4]]*(T1V-T2V),col="green")

\#\#\# Performance of Model 2

SSerr $12<-$

$\operatorname{sum}(($ model1_2\$coefficients[[1]]+model1_2\$coefficients[[2]]*W1V[1:100]+model1_2\$ coefficients[[3]]*W1V[1:100] $]^{\wedge} 2+$ model1_2\$coefficients[[4] $]^{*}(\mathrm{~T} 1 \mathrm{~V}[1: 100]-$

$\left.\mathrm{T} 2 \mathrm{~V}[1: 100])-\mathrm{T} 1 \mathrm{~V}[1: 100])^{\wedge} 2\right)$

MSE12<-SSerr12/n_data

n_regressor $<-3$ 
R_sqr12<-1-SSerr12/SStot1

R_sqr_adj12<-1-(1-R_sqr12)*(n_data-1)/(n_data-1-n_regressor)

AIC12<-log(MSE12)+(n_data $+2 *$ n_regressor $) / n \_$data

$\mathrm{AICc} 12<-\log (\mathrm{MSE} 12)+\left(\bar{n} \_\right.$data + n_regressor $) /($n_data-n_regressor-2)

BIC12 $<-\log (\mathrm{MSE} 12)+\mathrm{n} \_$regressor* $\log \left(\mathrm{n} \_\right.$data $) /$n_data

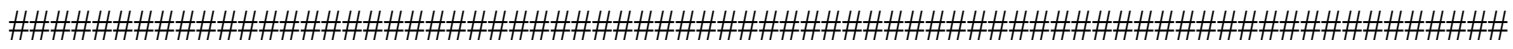

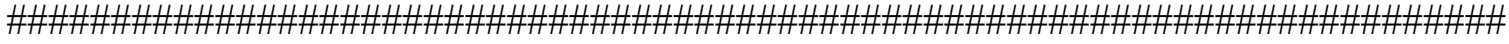
\#\#\#\#\#\#\#\#\#\#\#

\#\# ROOM 2

\#\#\# Room 2 - No Shared State Variables

\#\#\#Model $1=$ no shared state variable model

model2_1<-lm(T2 $\sim \mathrm{W} 2+\mathrm{I}(\mathrm{W} 2 \wedge 2))$

\#\#\#Plot results of model 1 against validation data

plot(Validation\$t,Validation\$TL2,type="l",col="red", ylim=c(265,340),xlab="Time

(s)",ylab="Temperature (K)",main="Room 2 (Red: Validation Data; Blue = No Shared

SV; Green: With Shared SV)")

lines(Validation\$t,model2_1\$coefficients[[1]]+model2_1\$coefficients[[2]]*W1V+model $2 \_1$ coefficients $[[3]] * \mathrm{~W} 1 \mathrm{~V}^{\wedge} 2$,col="blue")

\#\#\#Performance of Model 1

SSerr21<-

$\operatorname{sum}(($ model2_1\$coefficients[[1]]+model2_1\$coefficients[[2]]*W2V[1:100]+model2_1\$ coefficients[[3]]*W2V[1:100]^2-T2V[1:100])^2)

MSE21 <- SSerr21/n_data

n_regressor $<-2$

R_sqr21 $<-1$-SSerr21/SStot2

R_sqr_adj21<-1-(1-R_sqr21)*(n_data-1)/(n_data-1-n_regressor)

AIC21 $<-\log (\operatorname{MSE} 21)+($ n_data $+2 *$ n_regressor $) / n \_$data

AICc21 $<-\log (\mathrm{MSE} 21)+($ n_data + n_regressor $) /($ n_data-n_regressor-2)

BIC21 <- $\log (\mathrm{MSE} 21)+$ n_regressor* $\log ($ n_data $) /$ n_data

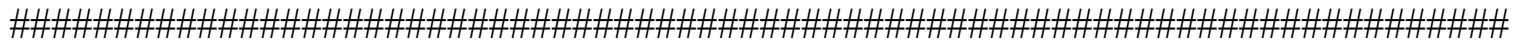
\#\#\#\#\#\# 
\#\#\# Room 2 - Shared State Variable $=$ T1 - T2

\#\#\#Model 2 = shared state variable model

model2_2<-lm(T2 $\left.\sim \mathrm{W} 2+\mathrm{I}(\mathrm{W} 2 \wedge 2)+\mathrm{DIFF} \_\mathrm{T}\right)$

\#\#\#Plot results of model 2 against validation data

lines(Validation\$t,model2_2\$coefficients[[1]]+model2_2\$coefficients[[2]]*W2V+model

$2 \_2 \$$ coefficients $[[3]]^{*} \mathrm{~W} 2 \overline{\mathrm{V}}^{\wedge} 2+$ model2_2\$coefficients[[4]]*(T1V-T2V),col="green")

\#\#\# Performance of Model 2

SSerr22 <-

$\operatorname{sum}((\operatorname{model} 22$ 2\$coefficients[[1]]+model2_2\$coefficients[[2]]*W2V[1:100]+model2_2\$

coefficients[[3]]*W2V[1:100]^2+model2_2\$coefficients[[4]]*(T1V[1:100]-

$\left.\mathrm{T} 2 \mathrm{~V}[1: 100])-\mathrm{T} 2 \mathrm{~V}[1: 100])^{\wedge} 2\right)$

MSE22 <- SSerr22/n_data

n_regressor $<-3$

R_sqr22 $<-1-$ SSerr22/SStot2

R_sqr_adj22<-1-(1-R_sqr22)*(n_data-1)/(n_data-1-n_regressor)

AIC22 $<-\log (\operatorname{MSE} 22)+($ n_data $+2 *$ n_regressor $) / n \_$data

AICc22 <- $\log (\operatorname{MSE} 22)+($ n_data + n_regressor $) /($ n_data-n_regressor-2)

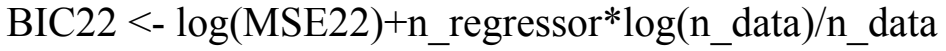




\section{Appendix II: Review of Statistical Performance Criteria}

Mean Squared Error (MSE)

Mean Squared Error measures the average of the squares of errors (i.e., amounts by which the predicted value differs from the true value). MSE is calculated as follows:

$M S E=\frac{\sum_{i}\left(\hat{y}_{i}-y_{i}\right)^{2}}{n}$

where:

$\mathrm{n}$ : Number of response data points

$\mathrm{y}:$ True value of response data

$\hat{y}$ : Predicted value of response data

Coefficient of Determination $\left(\mathbf{R}^{2}\right)$

Coefficient of Determination $\left(\mathrm{R}^{2}\right)$ is a measure that indicates the proportion of variability in a data set that is accounted for by the statistical model. In essence, $\mathrm{R}^{2}$ provides a measure of how likely future outcomes are to be predicted by the model. $\mathrm{R}^{2}$ is calculated as follows:

$R^{2}=1-\frac{S S_{e r r}}{S S_{t o t}}=1-\frac{\sum_{i}\left(y_{i}-\hat{y}_{i}\right)^{2}}{\sum_{i}\left(y_{i}-\bar{y}\right)^{2}}$

where:

$\mathrm{y}:$ True value of response data

$\hat{y}$ : Predicted value of response data

$\bar{y}:$ Mean of observed data 


\section{Adjusted Coefficient of Determination (Adjusted $\mathbf{R}^{2}$ )}

Adjusted Coefficient of Determination $\left(\mathrm{R}^{2}\right)$ adjusts $\mathrm{R}^{2}$ to account for the number of explanatory variables in a statistical model. Adjusted $\mathrm{R}^{2}$ increases only if a new variable improves the model more than would be expected by chance. Adjusted $\mathrm{R}^{2}$ can be negative and it is always less than or equal to $\mathrm{R}^{2}$. Adjusted $\mathrm{R}^{2}$ is calculated as follows:

$\bar{R}^{2}=1-\left(1-R^{2}\right) \frac{n-1}{n-p-1}$

where:

p: Total number of regression terms in a linear model, without the constant term

$\mathrm{n}$ : Number of response data points

$\mathrm{R}^{2}$ : Coefficient of determination

\section{Akaike Information Criterion (AIC)}

Akaike Information Criterion (AIC) is a measure of the relative goodness of fit of a statistical model. It can describe the tradeoff between bias and variance in model construction (i.e., the accuracy and complexity of a model). The measure on its own says nothing about how well a model fits the data in an absolute sense. Hence, AIC is only used for comparing multiple statistical models. AIC is calculated as follows:

$A I C=\log (M S E)+\frac{n+2 p}{n}$

where:

p: Total number of regression terms in a linear model, without the constant term

$\mathrm{n}$ : Number of response data points

MSE: Mean Squared Error 


\section{Bias Corrected Akaike Information Criterion (AICC)}

Bias Corrected Akaike Information Criterion (AICc) is a modification of the AIC which adds a correction for finite sample sizes. In other words, AICc adds a penalty to models with extra parameters. Just like the AIC, AICc cannot say in absolute terms how well a models fits a set of data, but it is used only for comparative purposes. It is recommended to use AICc when $n$ is small or $p$ is large. AICc is calculated as follows:

$$
A I C c=\log (M S E)+\frac{n+p}{n-p-2}
$$

where:

p: Total number of regression terms in a linear model, without the constant term

$\mathrm{n}:$ Number of response data points

MSE: Mean Squared Error

\section{Bayesian Information Criterion (BIC)}

Bayesian Information Criterion (BIC) is a measure of the relative fit of a statistical model that introduces a penalty term for the number of parameters in a model. The penalty term in BIC is larger than the one in AIC. Just like the AIC and AICc, the BIC says nothing about how well a model fits the data in absolute terms, but it is used only for comparing multiple statistical models. BIC is calculated as follows:

$B I C=\log (M S E)+\frac{p \log (n)}{n}$

where:

p: Total number of regression terms in a linear model, without the constant term

$\mathrm{n}$ : Number of response data points

MSE: Mean Squared Error 


\section{Appendix III: Bridge System of Systems Meta-Model Supporting Databases}

\section{National Bridge Inventory}

The National Bridge Inventory (NBI) is a database compiled by the Federal Highway Administration (FHWA). The NBI Database is the most extensive repository of data on highway bridges in the United States, and it was initiated in 1972 for the purpose of maintaining a unified database for bridges, including identification information, bridge types and specifications, operational conditions, bridge data including geometric data and functional description, inspection data, etc. This database currently includes detailed historical data on over 600,000 bridges. The data is collected by States and bridge owners as part of the comprehensive bridge inspection program established about 30 years ago. This information is reported to the Federal government and stored within the NBI. The data is primarily utilized for fund apportionment and cost allocation (Chase et al., 1999; US DOT 1995). The NBI contains information on the condition and load ratings, structure type and materials used, and average annual daily traffic, in addition to a variety of other data.

\section{National Climatic Data Center}

The United States National Climatic Data Center (NCDC) is the world's largest active archive of weather data. The Center contains an abundance of weather related data (added daily) that has been collected over the past 150 years. NCDC includes, among other, 99\% of all National Oceanic and Atmospheric Administration (NOAA) archives. NCDC receives data from a variety of sources, including, satellites, radar, automated airport weather stations, National Weather Services observers, aircraft, ships, radiosondes, wind profilers, rocketsondes, solar radiation networks, and National Weather Services Forecast/Warnings/Analyses Products. The Center's vast historical archives enable one to obtain a historical perspective on climate and climate change, and this is vital in studying global climate change and other environmental issues. The 
data stored by the Center can be used in analyses by industry, agriculture, science, hydrology, transportation, engineering and recreation (Wikipedia: National Climatic Data Center; Chase et al., 1999). NCDC enables us to obtain information on regional and local precipitation patterns, freeze-thaw cycles, and temperature ranges.

\section{Safe Accountable Flexible Efficient Transportation Equity Act (SAFETEA) Funding Tables}

Through the Safe Accountable Flexible Efficient Transportation Equity Act, FHWA collects and disseminates data on infrastructure apportionments of Federal-aid highway program funds to states for various transportation programs, including highway bridges (US DOT SAFETEA-LU). SAFETEA Funding Tables include SAFETEA-LU authorizations, apportionments, allocations, and obligation limitations for fiscal years $2005-2011$.

\section{Weigh-in-motion data (WIM)}

Weigh-in-motion data is captured by devices that are built in a road, and designed to capture truck axle weights and gross vehicle weights. This data is recorded as trucks drive over a measurement site at a weighting station. The WIM data collection efforts were initiated in the U.S. in the 1950s. The purpose of WIM data is to provide more realistic forecasts of truck traffic loads on road infrastructures.

\section{Bureau of Economic Analysis (BEA)}

The Bureau of Economic Analysis (BEA) is an agency of the U.S. Department of Commerce. Along with the Census Bureau and STAT-USA, it is a part of the Commerce Department's Economics and Statistics Administration. BEA produces economic accounts statistics that enable government, researchers and industries to model and better understand the performance of the U.S. economy. "BEA prepares national, regional, industry, and international accounts that present essential information on such key issues as economic growth, regional economic development, inter-industry relationships, and the Nation's position in the world economy" 
(BEA - Mission, Vision, Values). BEA collects and provides information on GDP and personal income, fixed assets, input-output demands between industries, international transactions, regional GDP, regional personal income, etc. Every five years the BEA collects information on the industry economic accounts. These are presented as input-output tables and as annual outputs by each industry. As such, these accounts provide a "detailed view of the interrelationships between U.S. producers and users and the contribution to production across industries. These accounts are used extensively by policymakers and businesses to understand industry interactions, productivity trends, and the changing structure of the U.S. economy" (BEA - About Industry).

\section{United States Census Bureau}

Census Bureau is a part of the U.S. Department of Commerce, and it provides quality data about the U.S. people and economy. In particular, the Bureau collects and disseminates Population and Housing Census every ten years, Economic Census every five years, Census of Governments every five years, American Community Surveys every year, various additional demographic and economic surveys and Economic Indicators (U.S. Census - About Us). Among other data, the Census Bureau collects information on the journey to work data (U.S. Census - Commuting). This survey provides information on commuting characteristics like the modes of transportation, travel time, travel distance, travel cost, etc.

\section{Bureau of Labor Statistics (BLS)}

"The Bureau of Labor Statistics of the U.S. Department of Labor is the principal Federal agency responsible for measuring labor market activity, working conditions, and price changes in the economy. Its mission is to collect, analyze, and disseminate essential economic information to support public and private decision-making" (BLS - About BLS). The BLS collects and provides information about inflation and prices, employment and unemployment characteristics, pay and benefit characteristics, time usage data, productivity, international labor comparisons, etc. In cooperation with the Census Bureau, the BLS conducts an annual American Time Use Survey (BLS - Time Use Survey), which reports the number of hours that Americans spend on primary 
activities (by age and gender). Additionally, in collaboration with the U.S. Census Bureau, the BLS conducts a Consumer Expenditure Survey (BLS - Consumer Expenditure Survey), which provides information on the spending habits of American consumers.

\section{Bureau of Transportation Statistics (BTS)}

The Bureau of Transportation Statistics was established in 1991 under the Intermodal Surface Transportation Efficiency Act (ISTEA) to collect, analyze and report transportation related information. In 2005 BTS became a part of the Research and Innovative Technology Administration (RITA). BTS collects and disseminates statistical information on multiple transportation modalities. BTS administers the Commodity Flow Surveys, which represent a "primary source of national and state-level data on domestic freight shipments by American establishments in mining, manufacturing, wholesale, auxiliaries, and selected retail industries" (BTS - Commodity Flow Survey). Commodity Flow Survey is conducted every five years and it includes information on the types, origins and destinations, dollar values, weights, modes of transportation, and distance of shipped commodities.

\section{National Household Travel Survey (NHTS)}

The U.S. Department of Transportation conducts the National Household Travel Survey to assess the mobility of the U.S. travelers, and to link travel related information to demographic, geographic, and economic data for analysis. This data collection is sponsored by the U.S. Bureau of Transportation Statistics (BTS) and the Federal Highway Administration (FHWA). The collected data is widely used in transportation and urban policy and planning. The survey gathers a wide variety of information including most commonly used modes of transportation, average trip durations, average trip distances, trip purposes, etc. The most recent survey was performed in 2009. It includes a total of 150,000 surveyed households (300,000 surveyed individuals). The 2009 NHTS includes a number of newly collected items like, employment questions relating to the worker's ability to set or alter their work schedules, worker's ability to telecommute, frequency of working from home; internet purchases, and safe routes to schools. The public use 
data from the 2009 NHTS is available at http://nhts.ornl.gov. For more information please see US DOT (2003) and Santos et al. (2011).

\section{Freight Analysis Framework}

The Federal Highway Administration of the U.S. Department of Transportation has created a Freight Analysis Framework (FAF) that "integrates data from a variety of sources to create a comprehensive picture of freight movement among states and major metropolitan areas by all modes of transportation" (US DOT - Freight Analysis Framework). FAF version $3\left(\right.$ FAF $\left.^{3}\right)$

provides information on tonnage, value and domestic ton-mileage by region of origin and destination, commodity type, transportation mode for year 2007, in addition to forecasted estimates through year 2040. 


\begin{tabular}{|c|c|}
\hline Database & Sample of available data \\
\hline $\begin{array}{l}\text { Bureau of Economic Analysis } \\
\text { (BEA) }\end{array}$ & $\begin{array}{c}\text { workforce numbers at state-wide and district levels - at } \\
\text { statewide level numbers broken down by sector; Local } \\
\text { Earnings and Employment Data }\end{array}$ \\
\hline $\begin{array}{l}\text { Bureau of Labor Statistics } \\
\text { (BLS) - Time Use Survey }\end{array}$ & $\begin{array}{c}\text { percent of civilian population engaging in each primary } \\
\text { activity - averages per day per gender (hours and } \\
\text { percent) }\end{array}$ \\
\hline $\begin{array}{l}\text { Bureau of Labor Statistics } \\
\text { (BLS) - Consumer } \\
\text { Expenditure Survey }\end{array}$ & $\begin{array}{l}\text { annual average expenditures divided among different } \\
\text { categories (e.g., housing, entertainment, health, food) }\end{array}$ \\
\hline $\begin{array}{c}\text { Bureau of Labor Statistics } \\
\text { (BLS) - VA Beach area } \\
\text { employment }\end{array}$ & $\begin{array}{l}\text { employment rates for different industry sectors (nonfarm } \\
\text { payrolls) }\end{array}$ \\
\hline $\begin{array}{c}\text { Bureau of Labor Statistics } \\
\text { (BLS) - VA Beach } \\
\text { compensation data } \\
\end{array}$ & $\begin{array}{l}\text { mean hourly earnings for full-time and part-time workers, } \\
\text { by occupation }\end{array}$ \\
\hline $\begin{array}{c}\begin{array}{c}\text { Bureau of Labor Statistics } \\
\text { (BLS) - VA Beach economic } \\
\text { indicators }\end{array} \\
\end{array}$ & $\begin{array}{l}\text { unemployment levels; nonfarm payroll employment; } \\
\text { mean hourly earnings for specific occupations }\end{array}$ \\
\hline \begin{tabular}{|c|}
$\begin{array}{c}\text { Census Bureau - Journey to } \\
\text { work data }\end{array}$ \\
\end{tabular} & $\begin{array}{c}\text { number of workforce commuting between different } \\
\text { regions }\end{array}$ \\
\hline $\begin{array}{l}\text { Census Bureau - VA Beach } \\
\text { people and business quick } \\
\text { facts }\end{array}$ & $\begin{array}{c}\text { population by: age, gender, race, education; number of } \\
\text { households; household income; per-capita income; total } \\
\text { number of firms; total amount of manufacturers } \\
\text { shipments; retail sales }\end{array}$ \\
\hline $\begin{array}{l}\text { Bureau of Transportation } \\
\text { Statistics (BTS) - National } \\
\text { Household Travel Survey }\end{array}$ & $\begin{array}{c}\text { average duration of trip by trip purpose and mode; } \\
\text { percentage of employees with flexible arrival time and } \\
\text { telecommuting option; division of trips by gender and } \\
\text { age; daily person miles travele;, distribution of workers } \\
\text { by commute mode }\end{array}$ \\
\hline $\begin{array}{l}\text { Bureau of Transportation } \\
\text { Statistics (BTS) - } \\
\text { Commodity Flow Survey }\end{array}$ & $\begin{array}{c}\text { national and state-level data on domestic freight } \\
\text { shipments: commodity type, origin and destination, } \\
\text { commodity weight, commodity value, modes of transport }\end{array}$ \\
\hline $\begin{array}{l}\text { Hampton Roads Travel } \\
\text { Survey }\end{array}$ & cities traveled to and reasons for traveling to city \\
\hline $\begin{array}{c}\text { Federal Highway } \\
\text { Administration (FHWA) - } \\
\text { National Bridge Inventory } \\
\text { (NBI) }\end{array}$ & $\begin{array}{c}\text { information on each U.S. bridge, including condition } \\
\text { rating, load rating, annual average daily traffic, structural } \\
\text { details }\end{array}$ \\
\hline $\begin{array}{c}\text { Federal Highway } \\
\text { Administration (FHWA) - } \\
\text { Freight Analysis Framework } \\
\left(\mathrm{FAF}^{3}\right) \\
\end{array}$ & $\begin{array}{l}\text { regional and national commodity movements by truck: } \\
\text { type of commodity, weight of commodity, } \$ \text { value of } \\
\text { commodity - data forecasted until } 2040\end{array}$ \\
\hline $\begin{array}{l}\text { Hampton Roads Planning } \\
\text { District Commission: } \\
\text { Intermodal Management } \\
\text { System - Regional Freight } \\
\quad \text { Study } 2007 \\
\end{array}$ & $\begin{array}{c}\text { percentage of freight transportation passing the HRBT; } \\
\text { locations of warehouse distribution centers in Virginia; } \\
\text { type, weight and \$ value of commodities coming into or } \\
\text { going out of the Hampton Roads region - data forecasted } \\
\text { until } 2035\end{array}$ \\
\hline Weigh-in-motion data (WIM) & truck axle weights, gross vehicle weights \\
\hline
\end{tabular}

Table 5: Sample data that is available from the cross-disciplinary databases, and that can be utilized to model the bridge infrastructure system of systems from multiple perspectives 
(This page intentionally left blank) 


\section{Bibliography}

Aktan, A.E., and D. Faust. A holistic integrated systems approach to assure the mobility, efficiency, safety and integrity of highway transportation. Published in "Structural Health Monitoring and Intelligent Infrastructure - Volume 1," Eds. Z. Wu and M. Abe. Sweets and Zeitlinger, Lisse, (2003): 7-19.

American Society of Civil Engineers, 1998. Report Card for America's Infrastructure (1998 Report Card). Available online:

http://www.asce.org/reportcard/index.cfm?reaction=full\&page=1.

American Society of Civil Engineers, 2003. Report Card for America's Infrastructure (2003 Report Card). Available online:

http://www.asce.org/reportcard/index.cfm?reaction=full\&page=6.

American Society of Civil Engineers, 2005. Report Card for America's Infrastructure (2005 Report Card). Available online:

http://www.asce.org/reportcard/2005/index2005.cfm.

American Society of Civil Engineers, 2009a. Report Card for America's Infrastructure (2009 Report Card). Available online: http://www.asce.org/reportcard/.

American Society of Civil Engineers, 2009b. America's Infrastructure Crisis: Can we Come Back From the Brink?. Available online: http://www.asce.org.

American Society of Civil Engineers (ASCE), 2011. Failure to Act: The Economic Impact of Current Investment Trends in Surface Transportation Infrastructure. Available at: http://www.asce.org/economicstudy/.

American Society of Civil Engineers (ASCE), 2013. Report Card for America's Infrastructure (2013 Report Card). Available online:

http:/www.infrastructurereportcard.org/. 
Amin, M.. National Infrastructures as Complex Interactive Networks. Chapter 14 in "Automation, Control, and Complexity: An Integrated Approach," Eds. Samad and Weyrauch, John Wiley and Sons, (2000): 263-286.

Andrijcic, E., Y.Y. Haimes, and T. Beatley. Public Policy Implications of Harmonizing Engineering Technology With Socio-Economic Modeling: Application to Transportation Infrastructure Management. Transportation Research Part A: Policy and Practice, Vol. 50, (2013): 62-73.

Andrijcic, E., S. Chase, Z. Guo and S. N. Hwang. Exploring system interdependencies via a multi-disciplinary modeling approach: application to bridge management. Proceedings of the 6th International Conference on Bridge Maintenance, Safety and Management (IABMAS), Lake Maggiore, Italy, July 8-12, 2012.

Asbeck, E., and Y.Y. Haimes. The partitioned multiobjective risk method. Large Scale Systems, Vol. 6, No. 1, (1984): 13-38.

Aschauer, D.A. Is public expenditure productive? Journal of Monetary Economics 23, no. 2 (March 1989): 177-200.

Axtell, R.. Why Agents? On the Varied Motivations for Agent Computing in the Social Sciences. Center on Social and Economic Dynamics, Working Paper No. 17 (2000).

Babcock, M. W., M. J. Emerson, and M. Prater. 1997. "A Model-Procedure for Estimating Economic Impacts of Alternative Types of Highway Improvement." Transportation Journal, Vol. 36, No. 4 (Summer), pp. 30-44.

Ben-Haim, Y. Doing Our Best: Optimization and the Management of Risk. Risk Analysis, Vol. 32, No. 8, (2012): $1326-1332$.

Bjornsson, H.C., I.M. de la Garza, and M.J. Nasir. A decision support system for road maintenance budget allocation. Computing in Civil and Building Engineering: Proceedings of the Eight International Conference. Palo Alto, CA. (2000): 702 - 709.

Blum, U. (1982): Effects of transportation investments on regional growth: a theoretical and empirical investigation. Papers of the Regional Science Association 49, 169-184. 
Boardman, J., and B. Sauser, System of Systems - the meaning of of. 2006 IEEE/SMC International Conference on System of Systems Engineering, Los Angeles, April 2426, 2006.

Bröcker, J. et al., "Methodology for the Assessment of Spatial Economic Impacts of Transport Projects and Policies," Available at: https://eldorado.tudortmund.de/handle/2003/26581.

Bruijn, H. de, and P.M. Herder. System and Actor Perspectives on Sociotechnical Systems. Systems, Man and Cybernetics, Part A: Systems and Humans, IEEE Transactions, Vol. 39, No. 5, (2009): 981-992.

Building America's Future Educational Fund. 2011. Building America's Future: Falling Apart and Falling Behind." Available online at: http://www.bafuture.org.

Bureau of Economic Analysis (BEA), Department of Commerce. Mission, Vision, Values. Available online at: http://www.bea.gov/about/mission.htm.

Bureau of Economic Analysis (BEA), Department of Commerce. About Industry. Available online at: http://www.bea.gov/about/overview_industry.htm.

Bureau of Labor Statistics (BLS), U.S. Department of Labor. About BLS. Available online at: http://www.bls.gov/bls/infohome.htm.

Bureau of Labor Statistics (BLS), U.S. Department of Labor. Consumer Expenditure Survey. Available online at: http://www.bls.gov/cex/.

Bureau of Labor Statistics (BLS), U.S. Department of Labor. American Time Use Survey. Available online at: http://www.bls.gov/tus/.

Bureau of Transportation Statistics (BTS), Research and Innovative Technology Administration. Commodity Flow Survey. Available online at: http://www.bts.gov/publications/commodity_flow_survey/.

Caballero, R., T. Gómez, M. Luque, F. Miguel, and F. Ruiz. Hierarchical generation of Pareto optimal solutions in large-scale multiobjective systems. Computers \& Operations Research, Vol. 29, (2002): 1537-1558. 
Caldwell, K. The Cost of Doing Nothing. Science, Vol. 334, No. 21, (2011): 289.

Chase, S., E.P. Small, and C. Nutakor. An In-Depth Analysis of the National Bridge Inventory Database Utilizing Data Mining, GIS and Advanced Statistical Methods. TRB Transportation Research Circular 498. 1999.

Chase, S., and L. Gáspár. Modeling the Reduction in Load Capacity of Highway Bridges With Age. Journal of Bridge Engineering, Vol. 5, No. 4, (2000): 331- 336.

Chasey, A.D., J.M. de la Garza, and D. Drew. Comprehensive level of service: Needed approach for civil infrastructure systems. Journal of Infrastructure Systems, Vol. 3, No. 4, (1997): 143 - 153.

Chasey, A.D., J.M. de la Garza, and D.R. Drew. Using simulation to understand the impact of deferred maintenance. Computer-Aided Civil and Infrastructure Engineering, Vol. 17, (2002): 269 - 279.

Chen, C. Linear System Theory and Design, 3rd edition. New York, NY: Oxford University Press, (1999).

Cho, S., P. Gordon, J.E. Moore, H.W. Richardson, M. Shinozuka. S. E. Chang. Integrating Transportation Network and Regional Economic Models to Estimate the Costs of a Large Earthquake. Final Report to the National Science Foundation (1999). Available at: http://www.usc.edu/schools/price/eqloss/nsfreport.pdf.

Chowdhury, M.A., P. Tan and S.L. William. An Interactive Multiobjective Decision Support Framework for Transportation Investment. Midwest Regional University Transportation Center Technical Report. December 2002. Available at: http://minds.wisconsin.edu/handle/1793/6913.

Dannin, E. (2011). Crumbling Infrastructure, Crumbling Democracy: Infrastructure Privatization Contracts and Their Effects on State and Local Governance. Northwestern Journal of Law and Social Policy, Vol. 6.

De la Barra, T.. "Integrated Land Use and Transport Modeling."Cambridge University Press, Cambridge, England (1989). 
De la Garza, J.M., D.R. Drew, and A.D. Chasey. Simulating highway infrastructure management policies. Journal of Management in Engineering, Vol. 14, No. 5, (1998): $64-72$.

DeLaurentis, D., and R.K. Callaway. A System-of-Systems Perspective for Public Policy Decisions. Review of Policy Research, Vol. 21, No. 6, (2004): 829 - 837.

DeLaurentis, D.. Appropriate modeling and analysis for systems of systems: Case study synopses using a taxonomy. 2008 IEEE International Conference on System of Systems Engineering. June 2-4, 2008.

DeLaurentis, D.. Understanding Transportation as a System of Systems Problem. Published in "System of Systems Engineering," Ed. Mo Jamshidi, John Wiley \& Sons, (2009): 520-541.

De Moor,B., P. Van Overschee, and W. Favoreel. Algorithms for subspace state-space system identification: An overview. Chapter 6 in "Applied and Computational Control, Signals, and Circuits, Volume 1,” Ed. B. Datta, Birkhauser Boston, (1999): 271-335.

De Schutter, B.. Minimal state-space realization in linear system theory: An Overview. Journal of Computational and Applied Mathematics, Special Issue on Numerical Analysis in the 20th Century - Vol. I: Approximation Theory, Vol. 121, No. $1-2,(2000): 331-354$.

Dooley, K., and S. Corman. Agent-based, genetic and emergent computational models of complex systems. Published in "Encyclopedia of Life Support Systems (EOLSS)," Ed. L. D. Kiel. Oxford, U.K.: UNESCO/EOLSS Publishers, 2002.

Dong, X., M.E. Ben-Akiva, J.L. Bowman, and J.L. Walker. Moving from trip-based to activity-based measures of accessibility. Transportation Research Part A, Vol. 40, (2006): 163-180.

Dunker, F.K., and B.G. Rabbat. Assessing Infrastructure Deficiencies: The Case of Highway Bridges. Journal of Infrastructure Systems, Vol. 1, No. 2 (1995): 100-119.

Ettema, D., A. Borgers, and H. Timmermans. A competing risk hazard model of activity choice, timing, sequencing and duration. Transportation Research Record 1493, (1995): $101-109$. 
Farmer, J.D., and D. Foley. The economy needs agent-based modeling. Nature, Vol. 460, No. 7256, (2009): 685-686.

Federal Highway Administration (FHWA). Office of Planning, Environment, \& Realty (HEP). TRANSIMS. Available online at:

http://www.fhwa.dot.gov/planning/tmip/transims/.

Forkenbrock, D.J., and G.E. Weisbrod. National Cooperative Highway Research Program (NCHRP) Report 456: Guidebook for Assessing the Social and Economic Effects of Transportation Projects. 2001. Available at:

http://onlinepubs.trb.org/onlinepubs/nchrp/nchrp_rpt_456-a.pdf.

Forkenbrock, D., S. Benshoff, and G.E. Weisbrod. Assessing the Social and Economic Effects of Transportation Projects. NCHRP Web Document 31 (Project B25-19):

Contractor's Final Report. 2001. Available at:

http://onlinepubs.trb.org/onlinepubs/nchrp/nchrp_w31.pdf.

Forrer, J., J.E. Kee, K. E. Newcomer, and E. Boyer (2010). Public-Private Partnerships and the Public Accountability Question. Public Administration Review, Vol. 70, No. 3.

Forrester, J.W.. "Industrial dynamics.” NewYork: Wiley (1961).

Friedland, I.M., H. Ghasemi, and S. B. Chase. The FHWA Long-Term Bridge Performance Program, Federal Highway Administration, Turner-Fairbank Highway Research Center, McLean, Va, USA, 2007.

Fujii, S., R. Kitamura, and T. Monma. A study of commuters' activity patterns for the estimation of induced trips. Journal of Infrastructure Planning and Management 562, (1997): $109-120$

Fwa, T.F., and Sinha, K.C., An Aggregate Damage Approach for Highway Pavement Performance Analysis, FHWA/JHRP-87-15, Joint Highway Research Project, School of Civil Engineering, Purdue University, West Lafayette, (1987).

Gomide, F., and Y.Y. Haimes. The multiobjective, multistage impact analysis method: Theoretical basis. IEEE Transactions on Systems, Man, and Cybernetics SMC, Vol. 14, (1984): 89-98. 
Hagani, A., S, Y. Lee, and J. H. Byun. A system dynamics approach to land use/ transportation system performance modeling Part 1: Methodology. Journal of Advanced Transportation, Vol. 37, No. 1, (2003): 1 - 41.

Haimes, Y.Y. and K. Tarvainen. Hierarchical-multiobjective framework for large scale systems. In P. Nijkamp and J. Spronk (Eds), Multicriteria Analysis in Practice. Gower Press, London (1980).

Haimes, Y.Y.. Hierarchical holographic modeling. IEEE Transactions on Systems, Man, and Cybernetics, Vol. 11, No. 9, (1981): 606-617.

Haimes, Y.Y., K. Tarvainen, T. Shima, and J. Thadathil. Hierarchical Multiobjective Analysis of Large-Scale Systems. Hemisphere Publishing Company, 1990.

Haimes, Y.Y.. Phantom system models for emergent multiscale systems. Journal of Infrastructure Systems, Vol. 13, No. 2, (2007): 81-87.

Haimes, Y.Y.. Phantom system models for risk management of systems of systems. International Journal of System of Systems, Vol. 1, No. 1, (2008): 222 - 236.

Haimes, Y.Y.. "Risk modeling, assessment, and management - 3rd Edition." New York, NY: John Wiley \& Sons, Inc., 2009.

Haimes, Y.Y.. Modeling complex systems of systems with Phantom System Models. To appear in Systems Engineering: The Journal of the International Council on Systems Engineering (INCOSE), Vol. 15, No. 2, (2012).

Haimes, Y.Y., and C. Schneiter. Covey's seven habits and the systems approach. IEEE Transactions on Systems, Man, and Cybernetics, Vol. 26, No. 4, (1996): 483 - 487.

Haimes, Y.Y., E. Andrijcic, and Z. Guo. Demonstrating the Predominant Contributions of Shared State Variables in Modeling Interdependent Systems Through Phantom System Models. Working paper (2013).

Hall, A.D., III.. “Metasystems Methodology.” New York, NY: Pergamon (1989). 
Ham, H., T.J. Kim, and D.E. Boyce, "Economic impacts of transportation network changes: Implementation of a combined transportation network and input-output model," Papers in Regional Science, Vol. 81, No.2, (2002): 223-246.

Hamed, M.M., and F.L. Mannering. Modeling travelers' postwork activity involvement: toward a new methodology. Transportation Science, Vol. 27, No. 4, (1993): 381 394.

Hampton Roads Planning District Commission. Intermodal Management System Regional Freight Study. April 2007.

Handy, C. Balancing Corporate Power: A New Federalist Paper. Harvard Business Review, Vol. 70, No. 6, (1992).

Hirsch, M., J.N. Prashker, and M.E. Ben-Akiva. Day-of-the week models of shopping activity patterns. Transportation Research Record 1085, (1985): 63 - 69.

Hirsch, M., J.N. Prashker, and M.E. Ben-Akiva. Dynamic model of weekly activity pattern. Transportation Science, Vol. 20, No. 1, (1986): 24-36.

Istrate, E., R. Puentes, The Brookings Institution (2011). Moving Forward on Public Private Partnerships: U.S. and International Experience With PPP Units. Available online at:

http://www.brookings.edu/papers/2011/1208_transportation_istrate_puentes.aspx.

Jamshidi, M.. Introduction to System of Systems. Chapter 1 in "System of Systems Engineering: Innovations for the $21^{\text {st }}$ Century," Ed. M. Jamshidi, John Wiley \& Sons, (2009): 1 - 20.

Jiang, B. A Review of Studies on the Relationship between Transport Infrastructure Investments and Economic Growth, A Report for the Canada Transportation Act Review Panel, January 10, 2001.

Kahneman, D. (2011). Thinking Fast and Slow. New York: Farrar, Straus and Giroux.

Kim, K.. A transportation planning model for state highway management: A decision support system methodology to achieve sustainable development. Ph.D. Dissertation, Virginia Polytechnic Institute and State University, February 1998, Blacksburg, VA. 
Available online at: http://scholar.lib.vt.edu/theses/public/etd-11798183731/materials/etd.pdf.

Kim, E. Geoffrey Hewings, and Chowoon Hong, “An Application of an Integrated Transport Network- Multiregional CGE Model: a Framework for the Economic Analysis of Highway Projects," Economic Systems Research. Vol. 16, No. 3, (2004): 235-258.

Kitamura, R.. Generation of Synthetic Daily Activity-Travel Patterns: Outline of the Approach. A report prepared for the National Institute of Statistical Sciences and Los Alamos National Laboratory, Institute of Transportation Studies, University of California, Davis, California (1995).

Kitamura, R. Applications of models of activity behavior for activity based demand forecasting. TMIP (Travel model improvement program) Activity-based travel forecasting conference, June 1996. Available at: http://media.tmiponline.org/clearinghouse/abtf/kitamura.pdf.

Kitamura, R., T. Yamamoto, S. Fujii, and S. Sampath. A Discrete-Continuous Analysis of Time Allocation to Two Types of Discretionary Activities Which Accounts for Unobserved Heterogeneity. Published in "Transportation and Traffic Theory," Ed. Lesort, J.B.. Elsevier, Oxford, (1996): 431 - 453.

Klosterman, R.E. The What If? Collaborative Support System. Environment and Planning, B: Planning and Design. Vol. 26, (1999): 393-408.

Labi, S., and K. C. Sinha.The Effectiveness of Maintenance and Its Impact on Capital Expenditures.Publication FHWA/IN/JTRP-2002/27. Joint Transportation Research Program, Indiana Department of Transportation and Purdue University, West Lafayette, Indiana, 2003.

Lasdon, L.S., and J.D. Schoeffler. Decentralized Plan Control. ISA Transactions, Vol. 5, (1966): 175-183.

Leach, M.R., and Y.Y. Haimes. Multiobjective risk-impact analysis method. Risk Analysis, Vol. 7, No. 2, (1987): 225-241. 
Lewe, J., D. DeLaurentis, and D. Mavris. Foundation for Study of Future Transportation Systems Through Agent-Based Simulation. 24th International Congress of the Aeronautical Sciences (ICAS), Yokohama, Japan, August 2004.

Li, D. and Y.Y. Haimes. A hierarchical generating method with feasible decomposition. Proceedings of the 10 IFAC Triennial World Congress. Large Scale Systems: Multilevel Control. (1987): 73-78.

Ljung, L.. Perspectives on system identification. Annual Reviews in Control, Vol. 34, (2010): 1-12.

Lounis, Z., L. Daigle, D. Cusson, and H. Almansour. A Multi-Objective Approach for the Management of Aging Critical Highway Bridges. NRCC-51266. July 2009. Available at: http://www.nrc-cnrc.gc.ca/irc.

Madanat, S., and Mishalani, R., Selectivity Bias in Modeling Highway Pavement Maintenance Effectiveness. Transportation Research Record 1229, Transportation Research Board, Washington, D.C., (1995).

Maier, M.W.. Architecting principles for systems of systems. Systems Engineering, Vol. 1, No. 4, (1998): 267-284.

Malone, T.W. What is coordination theory. Working paper no. 2051-88. MIT Sloan School of Management, Cambridge, MA (1988).

Malone, T.W. and K. Crowston. The Interdisciplinary Study of Coordination. $A C M$ Computing Surveys, Vol. 26, No. 1, (1994): 87 - 199.

Mäkilä, P.M., J.R. Partington. On linear models for nonlinear systems. Automatica, Vol. 39, No. 1, (2003): 1-13.

Mäkilä, P.M., J.R. Partington. Least-squares LTI approximation of nonlinear systems and quasistationarity analysis. Automatica, Vol. 40, No. 7, (2004): 1157-1169.

Mäkilä, P.M.. On optimal LTI approximation of nonlinear systems. IEEE Transactions on Automatic Control, Vol. 49, No. 7, (2004): 1178-1182. 
Min, H., W. Beyeler, T. Brown, Y. J. Son, and A. T. Jones. Toward modeling and simulation of critical national infrastructure interdependencies. IEEE Transactions, Vol. 39, (2007): $57-71$.

Moran, M., and H. Shapiro. Fundamentals of engineering thermodynamics, $3^{\text {rd }}$ edition. New York: Wiley, 1996.

Mouaket, I.M., and Sinha, K.C., Cost Effectiveness of Routine Maintenance on Rigid Pavements, FHWA/JHRP-91-11, Joint Highway Research Project, School of Civil Engineering, Purdue University, West Lafayette (1991).

Munnell, A.H. Infrastructure Investment and Economic Growth. Journal of Economic Perspectives 6 (1992), no. 4: 189-98.

Nadiri, M.I. and Mamuneas, T.P., (1996), "Highway Capital and Productivity Growth", Eno Transportation Foundations, Inc. Lansdown, VA U.S.

National Cooperative Highway Research Program (NCHRP) (2001). NCHRP Report 456: Guidebook for Assessing the Social and Economic Effects of Transportation Projects.

National Cooperative Highway Research Program (NCHRP) (2010). NCHRP Synthesis 406: Advanced Practices in Travel Forecasting.

National Council on Public Works Improvement. Fragile Foundations: A Report on America's Public Works. Washington, DC: U.S. Government Printing Office (1988).

National Science Foundation (NSF). A report by the NSF-IRIS Review Panel for Research on Coordination Theory and Technology. NSFF Forms and Publication Unit, National Science Foundation, Washington, D.C. (1989).

Norfolk Branch of the American Society of Civil Engineers. Hampton Roads Transportation Report Card: Putting Our Cities to the Test. 2005. Available online at: https://apps.asce.org/reportcard/2005/assets/pdf/hampton_roads_report.pdf.

OECD - International Transport Forum. Joint Transport Research Centre Discussion Paper No. 2008-6. Round Table, 25-26 October 2007, Boston. (2008). Available 
online at:

http://www.internationaltransportforum.org/jtrc/discussionpapers/DP200806.pdf

Oum, T.H., W.G. Waters and Chunyan Yu, (1998), "Development of an Econometric Model Linking Public Transportation Investments to Economic Growth in British Columbia", The Centre for Transportation Studies, The University of British Columbia.

Ozbek, M. E., J.M. de la Garza, and K. Triantis. Data and Modeling Issues Faced during the Efficiency Measurement of Road Maintenance Using Data Envelopment Analysis. Journal of Infrastructure Systems, Vol. 16, No. 21, (2010): 21 - 30.

Parker, J.M.. Applying a System of Systems Approach for Improved Transportation. S.A.P.I.EN.S, Vol. 3, No. 3, (2010).

Parunak, H. Van Dyke, R. Savit, and R.L. Riolo. Agent-Based Modeling vs. EquationBased Modeling: A Case Study and Users ' Guide. Proceedings of Workshop on Modeling Agent Based Systems (MABS98), Paris, 1998.

Patidar, V., S. Labi, K.C. Sinha, and P. Thompson. National Cooperative Highway Research Program Report 590. Multi-Objective Optimization for Bridge Management Systems. 2007. Available at:

http://onlinepubs.trb.org/onlinepubs/nchrp/nchrp_rpt_590.pdf.

PlanetMath.org. General system definitions (version 28). Available at: http://planetmath.org/SystemDefinitions.html.

Puentes, R., The Brookings Institution (2009). Promises and Pitfalls in Public-Private Partnerships for Transportation. Available online at: http://www.brookings.edu/speeches/2009/0714_transportation_puentes.aspx.

Rabeau, S., P. Depince, F. Bennis. Collaborative optimization of complex systems: a multidisciplinary approach. International Journal of Interactive Design and Manufacturing, Vol. 1, (2007): 209-218.

Ramaswamy, R. and Ben-Akiva, M., Estimation of Highway Pavement Deterioration from In-Service Pavements Data, Transportation Research Record 1272, Transportation Research Board, National Research Council, Washington D.C., (1990). 
Rashid, M.M., and P. Herabat. Multiattribute Prioritization Framework for Bridges, Roadside Elements, and Traffic Control Device Maintenance. In Transportation Research Circular E-C128. (2008): 175 - 188.

Rephann, T. and A. Isserman, "New highways as economic development tools: An evaluation using quasi-experimental matching methods," Regional Science and Urban Economics 24, no. 6 (December 1994): 723-751.

Rietveld, P. and F. Bruinsma (1998), Is transport infrastructure effective? Transport infrastructure and accessibility: impacts on the space economy, Springer, Berlin

Rigole, T. and G. Deconinck. A Survey on Modeling and Simulation of Interdependent Critical Infrastructures. $3^{\text {rd }}$ IEEE Benelux Young Researchers Symposium in Electrical Power Engineering. Ghent, Belgium, 2006.

Rinaldi S., J. Peerenboom, and T. Kelly. Identifying, Understanding, and Analyzing Critical Infrastructure Interdependencies. IEEE Control Systems Magazine, IEEE, (2001): 11-25.

Roads to the Future. Hampton Roads Bridge-Tunnel. (2007) http://www.roadstothefuture.com/I64_VA_HRBT.html

Robert, W.E., A.R. Marshall, R.W. Shepard, and J. Aldayuz. Pontis Bridge Management System: State of the Practice in Implementation and Development. Transportation Research Circular E-C049. (2003): 49 - 60. Available at: http://pubsindex.trb.org/view.aspx?id=644472.

Sage, A.P. and C.D. Cuppan. On the Systems Engineering and Management of Systems of Systems and Federations of Systems. Information, Knowledge, Systems Management, Vol. 2, No. 4, (2001): 325-345.

Santos, A., N. McGuckin, H.Y. Nakamoto, D. Gray, and S. Liss. Summary of Travel Trends: 2009 National household Survey. Report No. FHWA-PL-11-022. 2011.

Schoukens, J., J.G. Nemeth, P. Crama, Y. Rolain, and R. Pintelon. Fast approximate identification of nonlinear systems. Automatica, Vol. 39, No. 7, (2003): 1267-1274. 
Shen, L.Y., Y.Z. Wu, E.H.W. Chan, and J.L. Hao. Application of system dynamics for assessment of sustainable performance of construction projects. Journal of Zhejiang University Science, Vol. 6, No. 4, (2005): 339 - 349.

Short, J. and A. Kopp, "Transport infrastructure: Investment and planning. Policy and research aspects,” Transport Policy 12, no. 4 (July 2005): 360-367.

Simon, H. A. Rational choice and the structure of the environment. Psychological Review, Vol. 63 No. 2, (1956): 129-138.

Singh, B. Interconnected Roles (IR): A coordinated model. Technical Report CT-84-92. Microelectronics and Computer Technology Corp, Austin, TX (1992).

Slovic, P. (2000). Perceived Risk, Trust and Democracy. In "The Perception of Risk." London: Earthscan.

Inalhan, G., Stipanovic, D.M., and C.J. Tomlin. Decentralized optimization, with application to multiple aircraft coordination. Proceedings of the $41^{\text {st }}$ IEEE Conference on Decision and Control, Vol. 1, (2002): 1147 - 1155.

Strauss, R. Road to Nowhere: Federal Transportation Infrastructure Policy. A report by Renewing America Publications at the Council on Foreign Relations. June 2012.

Tarvainen, K. and Y.Y. Haimes. Coordination of Hierarchical Multiobjective Systems: Theory and Methodology. IEEE Transactions on Systems, Man, and Cybernetics, Vol. SMC-12, No. 6, (1982): 751-764.

Tarvainen, K, Y.Y. Haimes, and I. Lefkowitz. Decomposition Methods in Multiobjective Discrete-time Dynamic Problems. Automatica, Vol. 19, No. 1, (1983): 15-28.

Tarvainen, K. On the generating of Pareto optimal alternatives in large scale systems. Proceedings of the Fourth IFAC Symposium on Large Scale Systems, Zurich, Switzerland, (1986): 461-466.

Tesfatsion, L.. Agent-Based Computational Economics: Growing Economies from the Bottom Up. Artificial Life, Vol. 8, (2002): 55-82. 
U.S. Census Bureau, U.S. Department of Commerce. About Us. Available online at: http://www.census.gov/aboutus/.

U.S. Census Bureau, U.S. Department of Commerce. Commuting (Journey to Work). Available online at: http://www.census.gov/hhes/commuting/.

U.S. Department of Transportation (US DOT) - Federal Highway Administration. Freight Analysis Framework. Available online at: http://www.ops.fhwa.dot.gov/freight/freight_analysis/faf/.

U.S. Department of Transportation (US DOT) - Federal Highway Administration. HERS-ST 2.0 Highway Economic Requirements System - State Version. 2002.

U.S. Department of Transportation (US DOT) - Federal Highway Administration. Highway Economic Requirements System - State Version Technical Report. 2005.

U.S. Department of Transportation (US DOT) - Federal Highway Administration. Safe Accountable Flexible Efficient Transportation Equity Act: A Legacy for Users. Available online at: http://www.fhwa.dot.gov/safetealu/index.htm.

U.S. Department of Transportation (US DOT) - Federal Highway Administration. Recording and Coding Guide for the Structure Inventory and Appraisal of the Nation's Bridges. Report No. FHWA-PD-96-001. December 1995. Available online at: http://www.fhwa.dot.gov/bridge/mtguide.pdf.

U.S. Department of Transportation (US DOT) - Bureau of Transportation Statistics. NHTS 2001 highlights Report, BTS03-05. 2003.

Wang, J., H. Lu, and H. Peng. System Dynamics Model of Urban Transportation System and Its Application. Journal of Transportation Systems Engineering and Information Technology, Vol. 8, No. 3, (2008): 83 - 89.

Wells, G.D., and A.P. Sage. Engineering a System of Systems. Published In "System of Systems Engineering,” Ed. Mo Jamshidi, John Wiley \& Sons, (2009): 44 - 76.

Weisbrod, G. Models to Predict the Economic Development Impact of Transportation Projects: Historical Experience and New Applications. Annals of Regional Science, Vol. 42, No. 3, (2008): 519-543. 
Weisbrod, G. and J. Beckwith (1992). "Measuring Economic Development Benefits for Highway Decision-making: The Wisconsin Case," Transportation Quarterly, Vol. 46, No. 1, pp. 57-79, January.

Wikepedia. National Climatic Data Center. Available online at: http://en.wikipedia.org/wiki/National_Climatic_Data_Center.

Zadeh, P.M., V.V. Toropov and A.S. Wood. Metamodel-based collaborative optimization framework. Structural and Multidisciplinary Optimization, Vol. 38, (2009): 103-115.

Zavadskas, E.K., R. Liias, and Z. Turskis. Multi-Attribute Decision-Making Methods for Assessment of Quality in Bridges and Road Construction: State-of-the-Art Surveys. The Baltic Journal of Road and Bridge Engineering. Vol. 3, No. 3, (2008): 152 - 160.

Zhu, S., D. Levinson, H.X. Liu, and K. Harder. The traffic and behavioral effects of the I35W Mississippi River bridge collapse. Transportation Research Part A, Vol. 44, (2010): pp. 771-784. 